\title{
MÉTHODES GÉOMÉTRIQUES ET ANALYTIQUES POUR ÉTUDIER L'APPLICATION EXPONENTIELLE, LA SPHÈRE ET LE FRONT D'ONDE EN GÉOMÉTRIE SOUS-RIEMANNIENNE DANS LE CAS MARTINET
}

\author{
Bernard Bonnard ${ }^{1}$ et Monique Chyba $^{2}$
}

\begin{abstract}
Consider a sub-riemannian geometry $(U, D, g)$ where $U$ is a neighborhood of 0 in $\mathbf{R}^{3}, D$ is a Martinet type distribution identified to $\operatorname{ker} \omega, \omega$ being the 1-form: $\omega=d z-\frac{y^{2}}{2} d x, q=(x, y, z)$ and $g$ is a metric on $D$ which can be taken in the normal form: $g=a(q) d x^{2}+c(q) d y^{2}, a=1+y F(q), c=1+G(q)$, $G_{\left.\right|_{x=y=0}}=0$. In a previous article we analyze the flat case: $a=c=1$; we describe the conjugate and cut loci, the sphere and the wave front. The objectif of this article is to provide a geometric and computational framework to analyze the general case. This frame is obtained by analysing three one parameter deformations of the flat case which clarify the role of the three parameters $\alpha, \beta, \gamma$ in the gradated normal form of order 0 where: $a=(1+\alpha y)^{2}, c=(1+\beta x+\gamma y)^{2}$. More generally this analysis provides an explanation of the role of abnormal minimizers in SR-geometry.

Résumé. Considérons un problème sous-riemannien local $(U, D, g)$ où $U$ désigne un voisinage de 0 dans $\mathbf{R}^{3}, D$ est une distribution de type Martinet identifiée à ker $\omega$, où $\omega$ est la 1-forme : $\omega=d z-\frac{y^{2}}{2} d x$, $q=(x, y, z)$ et $g$ est une métrique sur $D$ dont une forme normale est : $g=a(q) d x^{2}+c(q) d y^{2}$, $a=1+y F(q), c=1+G(q), G_{\left.\right|_{x=y=0}}=0$. Dans un précédent article on a analysé le cas dit plat où $a=c=1$; on a décrit le lieu conjugué, le lieu de coupure, la sphère et le front d'onde. L'objectif de cet article est de fournir un cadre géométrique et calculatoire pour étudier le cas général. Ce cadre est obtenu à partir de l'analyse de trois déformations à un paramètre du cas plat, qui clarifie le rôle des trois paramètres $\alpha, \beta, \gamma$ dans la forme normale graduée d'ordre $0: a=(1+\alpha y)^{2}, c=(1+\beta x+\gamma y)^{2}$. Enfin cette étude fournit une bonne explication du rôle des géodésiques anormales minimisantes en géométrie SR.
\end{abstract}

AMS Subject Classification. 49J15, 53C22.

Received June 3, 1998. Revised March 19, 1999.

\section{INTRODUCTION}

Ce travail est le second d'une série d'articles consacrés à l'étude du problème sous-riemannien local $(U, D, g)$ où $U$ est un ouvert de $\mathbf{R}^{3}$ contenant $0, D$ est la distribution de type Martinet ker $\omega$ où $\omega$ est la 1 -forme $: d z-\frac{y^{2}}{2} d x$,

Keywords and phrases: Sub-riemannian geometry, Martinet case, abnormal geodesics, sphere and wave front of small radius. Mots clés : Géométrie sous-riemannienne, le cas Martinet, géodésiques anormales, sphère et front d'onde de petit rayon.

${ }^{1}$ Université de Bourgogne, Laboratoire de Topologie, UMR 5584 du CNRS, BP. 400, 21004 Dijon Cedex France; e-mail: bbonnard@u-bourgogne.fr

${ }^{2}$ Université de Paris VI, Case 247, 4 place Jussieu, 75252 Paris Cedex 05, France; e-mail: chyba@sunny.unige.ch 
$q=(x, y, z)$ désignant les coordonnées de $\mathbf{R}^{3}$ et $g$ une métrique sur $D$ qui s'écrit $a(q) d x^{2}+2 b(q) d x d y+c(q) d y^{2}$ où $a, b, c$ sont des germes en 0 de fonctions analytiques réelles. L'importance de cette étude réside dans le fait que c'est un modèle pour étudier le rôle des géodésiques minimisantes anormales en géométrie SR. La forme normale calculée dans [1] montre que l'on peut supposer que la métrique $g$ est une somme de carrés $a d x^{2}+c d y^{2}$ où de plus $a$ et $c$ admettent l'écriture suivante : $a=1+y F(q), c=1+G(q)$ où $G_{\left.\right|_{x=y=0}}=0$. Notre étude est localisée en 0 et en utilisant la procédure de graduation expliquée dans [20] il convient d'attribuer les poids suivants aux coordonnées : le poids de $x, y$ est 1 et le poids de $z$ est 3 . En utilisant cette graduation la forme normale d'ordre -1 est le cas plat où $g$ s'écrit $d x^{2}+d y^{2}$ et la forme normale d'ordre 0 est $g=(1+\alpha y)^{2} d x^{2}+(1+\beta x+\gamma y)^{2} d y^{2}$ et dépend de trois paramètres réels $\alpha, \beta, \gamma$. Dans notre premier article [1] on a fait une analyse quasi-exhaustive du cas plat et l'objectif de cet article est de faire une analyse du cas d'ordre 0 considéré comme une déformation du cas plat. Pour introduire précisément notre problème et formuler les résultats obtenus il est nécessaire de donner certaines définitions.

\subsection{Définitions et préliminaires}

Une courbe admissible est une courbe absolument continue $q:[0, T] \rightarrow \mathbf{R}^{3}$ telle que $\dot{q}(t) \in D(q(t)) \backslash\{0\}$ pour presque tout $t$. Notons (,) le produit scalaire défini par $g$. La longueur et l'énergie d'une courbe admissible sont données respectivement par :

$$
L(q)=\int_{0}^{T}(\dot{q}(t), \dot{q}(t))^{\frac{1}{2}} d t, \quad E(q)=\int_{0}^{T}(\dot{q}(t), \dot{q}(t)) d t .
$$

Soient $q_{0}, q_{1} \in U$, une courbe minimisante joignant $q_{0}$ à $q_{1}$ est une courbe admissible $q:[0, T] \rightarrow U, q(0)=$ $q_{0}, q(T)=q_{1}$ de longueur minimale. C'est un résultat classique que le problème de minimiser la longueur équivaut au problème de minimiser l'énergie. Pour calculer ces courbes minimisantes il est commode d'utiliser le principe du maximum de la théorie du contrôle. Selon ce principe les courbes minimisantes appartiennent à une famille restreinte de courbes que l'on calcule comme suit.

Soient $G_{1}, G_{2}$ deux champs de vecteurs analytiques définis sur $U$ tels que $D$ soit engendré par $G_{1}, G_{2}$. Introduisons alors le système :

$$
\frac{d q(t)}{d t}=\sum_{i=1}^{2} u_{i}(t) G_{i}(q(t))
$$

où $u(t)=\left(u_{1}(t), u_{2}(t)\right)$ est le contrôle associé à la courbe $q(t)$. Soit $\mu$ une constante égale à 0 ou $\frac{1}{2}$ et considérons le pseudo-hamiltonien :

$$
H_{\mu}(q, p, u)=\langle p, G(q) u\rangle-\mu(G(q) u, G(q) u)
$$

où $p=\left(p_{x}, p_{y}, p_{z}\right)$ est le vecteur adjoint, $(p, \mu)$ est non nul, $\langle$,$\rangle est le produit scalaire usuel de \mathbf{R}^{3}$ et $G(q) u$ désigne $\sum_{i=1}^{2} u_{i} G_{i}(q)$. On appelle relèvement géodésique une courbe $(q, p)$ absolument continue définie sur $[0, T]$ telle que $t \mapsto(q(t), p(t))$ est solution presque partout des équations suivantes :

$$
\begin{gathered}
\frac{d q(t)}{d t}=\frac{\partial H_{\mu}}{\partial p}(q(t), p(t), u(t)), \quad \frac{d p(t)}{d t}=-\frac{\partial H_{\mu}}{\partial q}(q(t), p(t), u(t)) \\
\frac{\partial H_{\mu}}{\partial u}(q(t), p(t), u(t))=0 .
\end{gathered}
$$


Sa projection $t \mapsto q(t)$ sur $U$ s'appelle une géodésique. On peut observer qu'il y a deux types de relèvements géodésiques. Si $\mu=0$, le relèvement est dit anormal et si $\mu \neq 0$ il est dit normal et les géodésiques correspondantes sont qualifiées respectivement d'anormales et de normales. Une même géodésique peut admettre un relèvement normal et un relèvement anormal ; on dit qu'elle est strictement anormale si elle est la projection d'un relèvement anormal mais non d'un relèvement normal.

Pour calculer les géodésiques on procède ainsi. Dans le cas normal $\mu \neq 0$, l'équation dite de contrainte (1.3) est linéaire par rapport à $u$ et le contrôle correspondant à chaque trajectoire peut être calculé comme une application analytique $\hat{u}:(q, p) \in U \times \mathbf{R}^{3} \rightarrow \mathbf{R}^{2}$. En remplaçant $u$ par $\hat{u}$ dans $H_{\mu}$ où $\mu=\frac{1}{2}$, on définit un hamiltonien $H_{n}$ sur $U \times \mathbf{R}^{3}: H_{n}(q, p)=H_{\frac{1}{2}}(q, p, \hat{u})$. En utilisant (1.2) et (1.3) on observe alors qu'un relèvement normal $(q, p)$ est solution de l'équation différentielle hamiltonienne analytique suivante :

$$
\frac{d q}{d t}=\frac{\partial H_{n}}{\partial p}(q, p), \frac{d p}{d t}=-\frac{\partial H_{n}}{\partial q}(q, p)
$$

Pour calculer les relèvements géodésiques anormaux dans le cas Martinet on procède comme suit. Ils ne dépendent que de $D$ et l'équation (1.3) avec $\mu=0$ est :

$$
\left\langle p(t), G_{i}(q(t))\right\rangle=0, \quad i=1,2 .
$$

En dérivant deux fois cette équation par rapport à $t$, on obtient que les géodésiques anormales sont contenues dans l'ensemble :

$$
\operatorname{det}\left(G_{1}, G_{2},\left[G_{1}, G_{2}\right]\right)=0
$$

qui est le plan $y=0$ correspondant au lieu où $\omega$ n'est pas une forme de contact. Ce plan qui a donc une signification géométrique s'appelle le plan de Martinet. Dans ce plan, les géodésiques anormales sont les droites : $z=z_{0}$.

Supposons que les géodésiques sont paramétrisées par la longueur d'arc: $(\dot{q}, \dot{q})=1$. Fixons la condition initiale $q_{0}$ à 0 et soit $\left(q\left(t, p_{0}\right), p\left(t, p_{0}\right)\right)$ la trajectoire de (1.4) issue en $t=0$ de $q_{0}=0$ et de $p_{0}$. Elle est contenue dans le niveau d'énergie $H_{n}=\frac{1}{2}$. L'application exponentielle est l'application :

$$
\exp _{0}:\left(p_{0}, t\right) \mapsto q\left(t, p_{0}\right) .
$$

Son domaine est l'ensemble $C \times \mathbf{R}$ où $C$ est le cylindre : $H_{n}\left(0, p_{0}\right)=\frac{1}{2}$. Observons que ce cylindre est non compact. Le point $q_{1}$ est dit conjugué à 0 le long de la géodésique normale $q($.$) s'il existe \left(p_{0}, t_{1}\right), t_{1}>0$ tel que $q()=.\exp _{0}\left(p_{0},.\right), q_{1}=\exp _{0}\left(p_{0}, t_{1}\right)$ et l'application exponentielle n'est pas une immersion en $\left(p_{0}, t_{1}\right)$. Le lieu conjugué $C(0)$ est l'ensemble des premiers points conjugués le long de toutes les géodésiques normales issues de 0 . Considérons maintenant une géodésique $q($.$) normale ou anormale. Le premier point où q($.$) cesse$ d'être minimisante s'appelle un point de coupure et l'ensemble de tous ces points lorsque $q($.$) décrit l'ensemble$ des géodésiques issues de 0 s'appelle le lieu de coupure $L(0)$. La sphère sous-riemannienne de rayon $r>0$ et d'origine 0 est l'ensemble $S(0, r)$ des points dont la distance SR à 0 est $r$. Le front d'onde de rayon $r$ est l'ensemble $W(0, r)$ de l'extrémité des géodésiques de longueur $r$, issues de 0 . Dans notre travail notre étude est localisée en 0 . On suppose que $r$ est assez petit de sorte que tout point à distance $r$ de 0 peut être joint par une géodésique minimisante. De fait la sphère $S(0, r)$ est un sous-ensemble de $W(0, r)$.

\subsection{Introduction du feuilletage $(\mathcal{F})$}

Considérons la forme normale graduée d'ordre $0: g=a d x^{2}+c d y^{2}$ où $a=(1+\alpha y)^{2}, c=(1+\beta x+\gamma y)^{2}$. Alors $F_{1}=\frac{1}{\sqrt{a}}\left(\frac{\partial}{\partial x}+\frac{y^{2}}{2} \frac{\partial}{\partial z}\right)$ et $F_{2}=\frac{1}{\sqrt{c}} \frac{\partial}{\partial y}$ constituent une famille orthonormée que l'on complète par $F_{3}=\frac{\partial}{\partial z}$ pour former une base. Introduisons $P_{i}=\left\langle p, F_{i}(q)\right\rangle$ pour $i=1,2,3$. Le hamiltonien $H_{n}$ s'écrit alors $\frac{1}{2}\left(P_{1}^{2}+P_{2}^{2}\right)$ 
et les géodésiques normales sont les solutions des équations :

$$
\begin{aligned}
\dot{x} & =\frac{1}{\sqrt{a}} P_{1} \\
\dot{y} & =\frac{1}{\sqrt{c}} P_{2} \\
\dot{z} & =\frac{y^{2}}{2 \sqrt{a}} P_{1} \\
\dot{P}_{1} & =\frac{P_{2}}{\sqrt{a} \sqrt{c}}\left(y P_{3}-\frac{a_{y}}{2 \sqrt{a}} P_{1}+\frac{c_{x}}{2 \sqrt{c}} P_{2}\right) \\
\dot{P}_{2} & =-\frac{P_{1}}{\sqrt{a} \sqrt{c}}\left(y P_{3}-\frac{a_{y}}{2 \sqrt{a}} P_{1}+\frac{c_{x}}{2 \sqrt{c}} P_{2}\right) \\
\dot{P}_{3} & =0 .
\end{aligned}
$$

En introduisant les coordonnées : $P_{1}=\cos \theta, P_{2}=\sin \theta, P_{3}=\lambda$ ces équations définissent un feuilletage $(\mathcal{F})$ sur le cylindre $S^{1} \times \mathbf{R}=\left(\mathrm{e}^{i \theta}, \dot{\theta}\right)$ qui s'obtient en paramétrisant les solutions par $\tau$ où $\tau$ est donné par : $\sqrt{a} \sqrt{c} \frac{d}{d t}=\frac{d}{d \tau}$ et en les projetant sur le plan de phase $\left(\theta, \theta^{\prime}\right)$. Un calcul facile montre que $(\mathcal{F})$ est défini par l'équation :

$$
\theta^{\prime \prime}+\lambda \sin \theta+\alpha^{2} \sin \theta \cos \theta-\alpha \beta \sin ^{2} \theta+\beta \cos \theta \theta^{\prime}=0
$$

où ' désigne la dérivée par rapport à $\tau$. De plus si $q_{0}=0$, alors $y(0)=0$ et les géodésiques issues de 0 vérifient la contrainte $(\mathcal{C})$

$$
\theta^{\prime}(\tau)=(\alpha \cos \theta(\tau)-\beta \sin \theta(\tau))
$$

pour $\tau=0$.

\subsection{Rappels des propriétés du cas plat}

On rappelle brièvement les 5 propriétés essentielles du cas plat dont on se propose d'étudier la stabilité, voir [1] pour l'étude complète du cas plat.

1.3.1. Optimalité et rigidité de la trajectoire anormale $a: t \rightarrow( \pm t, 0,0)$

La projection des géodésiques correspondantes à $\lambda=0$ dans le plan $(x, y)$ sont les droites issues de 0 et sont donc globalement minimisantes. La géodésique anormale a la propriété de rigidité suivante : il n'existe pas de géodésique normale $e:[0, T] \mapsto(x(t), y(t), z(t))$ telle que $e(0)=0, y(T)=z(T)=0$ et Ime non contenue dans l'axe $O x$.

\subsubsection{Intégrabilité}

Le flot géodésique possède 3 intégrales premières : $p_{x}, p_{z}$ et $H$ et est intégrable par quadratures. Si $e$ : $[0, T] \mapsto(x(t), y(t), z(t))$ désigne une géodésique normale telle que $p_{z}=\lambda \neq 0$ et $\theta(0) \neq n \pi$ alors $e($.$) peut$ être paramétrisée avec les fonctions de Jacobi de première et de seconde espèce : sn, $\mathrm{cn}, \mathrm{dn}$ et $E$. La variable $t \mapsto y(t)$ oscille de façon périodique avec une période $\frac{4}{\sqrt{\lambda}} K(k)$ où $K(k)$ est l'intégrale complète de première espèce $\int_{0}^{\frac{\pi}{2}} \frac{d \varphi}{\sqrt{1-k^{2} \sin ^{2} \varphi}}$ où le module $k$ est fonction de $\theta(0)$. La projection de ces géodésiques dans le plan $\left(\theta, \theta^{\prime}\right)$ sont les trajectoires d'un pendule oscillant et les cas limites $k \rightarrow 0$ et $k \rightarrow 1$ décrivent respectivement le pendule linéarisé et le comportement des trajectoires au voisinage de la séparatrice. Dans cette projection la trajectoire anormale $a: t \mapsto( \pm t, 0,0)$ s'identifie aux positions d'équilibre $\theta=n \pi, \theta^{\prime}=0$, voir figure 1 . 


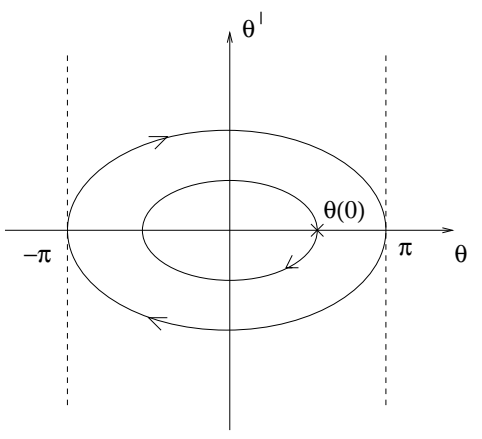

Figure 1. $(\lambda>0)$.
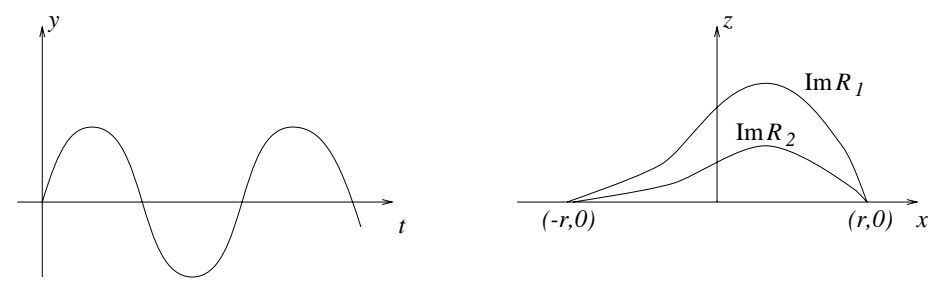

FiguRE 2

\subsubsection{Symétries, lieu conjugué et le lieu de coupure}

Dans le cas plat, le problème SR possède le groupe de symétries engendré par les deux difféomorphismes $S_{1}:(x, y, z) \mapsto(-x, y,-z)$ et $S_{2}:(x, y, z) \mapsto(x,-y, z)$. En particulier en utilisant $S_{1}$ on peut supposer que $\lambda \geq 0$. Du fait de la symétrie $S_{2}$, les deux géodésiques associées à $(\lambda, \theta(0))$ et $(\lambda,-\theta(0)), \lambda>0$, issues de 0 , se recoupent au même point et avec la même longueur à chaque $t_{n}=\frac{2 n}{\sqrt{\lambda}} K(k)$ où $k^{2}=\frac{1-\cos \theta(0)}{2}$. Le point de coupure d'une telle géodésique correspond précisément à la première intersection $t_{1}=\frac{2}{\sqrt{\lambda}} K(k)$ avec $y=0$ et le lieu de coupure $L(0)$ est le plan de Martinet $y=0$ moins la direction anormale : $O x$. L'intersection du lieu de coupure $L(0)$ et du lieu conjugué $C(0)$ est vide.

\subsubsection{Application retour et trace de la sphère et du front d'onde avec le plan de Martinet $y=0$}

On peut visualiser aisément à l'aide du feuilletage $(\mathcal{F})$ et de la contrainte $(\mathcal{C}): \dot{\theta}=0$ pour $y=0$ la trace de la sphère et du front d'onde de rayon $r$ avec le plan de Martinet $y=0$. Dans le plan $(x, z)$, les extrémités $( \pm r, 0)$ de la géodésique anormale appartiennent à la sphère. Sinon on peut supposer $\lambda>0$ et chaque point de $S(0, r) \cap\{y=0\}$ correspond à l'extrémité d'une géodésique de longueur $r$ à sa première intersection avec le plan $y=0$, i.e. avec $\dot{\theta}(r)=0$, les autres intersections formant le front d'onde. La trace de la sphère avec $y=0$ moins les points $( \pm r, 0)$ est donc l'image de l'application premier retour $R_{1}$ qui à une géodésique associe sa première intersection avec $y=0$. De façon plus générale l'application $n$-ième retour $R_{n}$ associe sa $n$-ième intersection. On représente sur la figure 2, une géodésique où $y$ varie périodiquement et l'image de l'application retour $R$. La trace de la sphère dans le domaine $z \geq 0$ avec le plan $y=0$ est $\operatorname{Im} R_{1} \cup( \pm r, 0)$. Le front d'onde est $\cup_{n \geq 1} \operatorname{Im} R_{n} \cup( \pm r, 0)$ et est formé d'une infinité de courbes se ramifiant en $( \pm r, 0)$.

\subsubsection{Non propreté de l'application premier retour et transcendance de la sphère}

On représente sur la figure 3 pour $r>0$ fixé : $\frac{2}{\sqrt{\lambda}} K(k)$, le domaine de $R_{1}$ avec $\lambda>0$ et son image. Les orientations des courbes indiquent les correspondances respectives. 


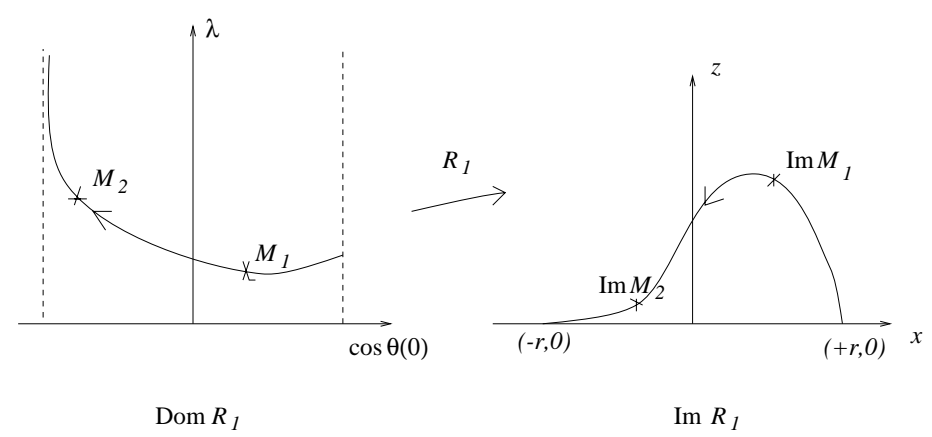

FiguRE 3

On observe que l'application $R_{1}$ n'est pas propre au voisinage du point $(-r, 0) \in \overline{\operatorname{Im} R_{1}}$. Par ailleurs lorsque $\theta(0) \rightarrow 0, k \rightarrow 0$ et $K(k) \rightarrow \frac{\pi}{2}$, soit $\sqrt{\lambda} \rightarrow \frac{\pi}{r}$ et lorsque $\theta(0) \rightarrow \pi, k \rightarrow 1$ et $K(k) \rightarrow-\log \sqrt{1-k^{2}}$ et la courbe $\operatorname{Dom} R_{1}$ possède une branche logarithmique. En utilisant alors la paramétrisation explicite des géodésiques on peut estimer aisément la transcendance de la sphère $S(0, r)$ au voisinage des points $( \pm r, 0)$.

$\mathrm{Au}$ voisinage du point $(r, 0)$, l'ensemble $\operatorname{Im} R_{1}$ est un ensemble analytique. Par contre au voisinage du point $(-r, 0)$ ce n'est pas le cas cet ensemble n'est pas sous-analytique. Par contre il appartient à la catégorie exp$\log$ et s'écrit localement comme un graphe : $z=F\left(\xi_{1}, \xi_{2}\right)$ où $F$ est un germe de fonction analytique en 0 et $\xi_{1}=X=\frac{x+r}{2}, \xi_{2}=\frac{\mathrm{e}^{-\frac{1}{\mathrm{X}}}}{\mathrm{X}^{2}}$ sont les facteurs d'échelle $\xi=\left\{\xi_{1}, \xi_{2}\right\}$.

\subsubsection{Conclusions tirées du cas plat}

Cette analyse montre les résultats fondamentaux suivants.

- Le calcul de la trace de la sphère avec le plan $y=0$ (et de la sphère dans son ensemble) se ramène à l'étude de l'application retour construite à partir du feuilletage $(\mathcal{F})$, et de la section définie par $(\mathcal{C})$ et la contrainte $t=r$. Le problème est donc similaire à l'évaluation de l'application retour de Poincaré-Dulac dans le calcul des trajectoires périodiques pour les équations différentielles. Ce problème a été abondamment étudié.

- Le comportement des géodésiques au voisinage de la géodésique anormale se ramène à l'étude des trajectoires du pendule au voisinage du pendule linéarisé et au voisinage de la séparatrice. En particulier la nature de la sphère au voisinage des points $( \pm r, 0,0)$ est liée à la propreté ou à la non propreté de l'application retour. On peut d'ailleurs observer qu'au point $(r, 0,0)$ la sphère reste sous-analytique bien que ce soit l'extrémité d'une géodésique anormale minimisante, voir les études de [5] et [17] pour des cas non minimisants.

\subsection{Problèmes du cas général}

L'étude du cas plat montre que l'on doit étudier en général les propriétés suivantes.

\subsubsection{Problème de rigidité}

D'un point de vue conceptuel il est important de formuler la propriété d'optimalité de la trajectoire anormale comme une conséquence de la propriété $(\mathcal{R})$ de rigidité géodésique suivante : $\forall M>0, \exists \varepsilon(M)$ tel que si $e$ : $[0, T] \mapsto(x(t), y(t), z(t))$ est une géodésique normale dont l'image n'est pas incluse dans l'axe $O x$ et sa longueur est inférieure à $M$ alors on n'a pas simultanément : $y(T)=z(T)=0$ si $|y| \leq \varepsilon(M)$. 


\subsubsection{Application retour dans le cas intégrable}

Dans le cas où $\beta=0,(\mathcal{F})$ est intégrable. On doit évaluer l'image de l'application retour au voisinage des points $( \pm r, 0)$. C'est un ensemble ramifié dont les branches doivent être calculées selon les techniques de géométrie analytique réelle sous la forme :

$(E L) \quad z=F\left(\xi_{1}, \xi_{2}, \ldots, \xi_{k}\right)$

où $F$ est le germe d'une fonction analytique et les $\xi_{i}$ sont des facteurs d'échelle dans la catégorie exp-log, [25].

\subsubsection{Application retour dans le cas général}

Dans le cas général le flot géodésique est non intégrable et le calcul de l'application retour pose deux problèmes :

1. Problème topologique. Ce problème est posé par l'existence dans le cas plat de géodésiques non issues de $\overline{0 \text { dont les projections }} \operatorname{sur}(\mathcal{F})$ sont des trajectoires d'un pendule en rotation et qui sont arbitrairement près de la séparatrice.

2. Problème asymptotique. On doit calculer des développements asymptotiques pour des solutions d'une équation différentielle au voisinage d'une séparatrice. Ce calcul est non classique.

\subsubsection{Trace du lieu de coupure et du lieu conjugué}

Dans le cas plat $L(0)$ est disjoint de $C(0)$ mais l'intersection de leurs fermetures contient la direction anormale $a: t \rightarrow( \pm t, 0,0)$. On doit étudier la stabilité de cette propriété.

L'objectif de ce travail est de donner une réponse partielle à ces problèmes. Notre étude est loin d'être exhaustive et on se limite essentiellement au cas intégrable. L'organisation de cet article est la suivante. Le rôle du paragraphe 2 est de rappeler la forme normale graduée d'ordre 0 introduite dans [1] et d'expliquer la signification géométrique des paramètres $\alpha, \beta, \gamma$ de cette forme. On donne ensuite deux formules pour calculer l'application exponentielle. La première est un développement formel utilisant la propriété de quasi-homogénéité du cas plat. Cette formule met en évidence le problème posé par l'existence de termes séculaires dans nos développements des solutions de $(\mathcal{F})$ au voisinage d'une séparatrice. Le second calcul n'est valide que lorsque la métrique $g$ est de la forme $a(y) d x^{2}+c(y) d y^{2}$ et l'équation des géodésiques normales est intégrable par quadratures. L'objectif du paragraphe 3 est d'analyser la déformation à un paramètre suivante du cas plat : $g=d x^{2}+(1+\gamma y)^{2} d y^{2}$ pour expliquer le rôle du paramètre $\gamma$ dans la forme normale. Pour ce type de déformation la géodésique anormale issue de 0 reste non strictement anormale comme dans le cas plat. On donne une paramétrisation des géodésiques en utilisant les fonctions de Jacobi. Cela permet d'analyser la sphère de petit rayon et notamment de représenter sa trace avec le plan de Martinet. L'objectif du paragraphe 4 est d'analyser le rôle du paramètre $\alpha$ dans la forme normale. Cette analyse est plus délicate que la précédente. En effet si $\alpha \neq 0$, la géodésique anormale issue de 0 devient strictement anormale et l'étude de la sphère au voisinage de cette direction est complexe. En particulier on est confronté à un problème d'instabilité lié à un phénomène de doublement de période. Ce problème est lié au problème topologique évoqué en section 1.4.3 qui est une conséquence du fait que $(\mathcal{F})$ est défini sur un cylindre et que dans le cas plat le pendule décrit par $(\mathcal{F})$ possède au voisinage de la séparatrice des trajectoires oscillantes et en rotation. Du point de vue de la géométrie analytique on verra aussi l'apparition du phénomène dit de doublement du logarithme dans le calcul de l'application retour. Dans le paragraphe 5 on étudie la déformation à un paramètre $g=d x^{2}+(1+\beta x)^{2} d y^{2}$. Si $\beta \neq 0$, l'équation des géodésiques n'est pas intégrable. On représente pour $\lambda>>\beta$ les trajectoires du feuilletage $(\mathcal{F})$ et en particulier on montre que ce système est sans feuille périodique non triviale. Une conséquence est de montrer que le front d'onde du cas plat est instable. La nature des singularités de $(\mathcal{F})$ conduit par ailleurs à conjecturer que la sphère n'est pas sous-pfaffienne. Enfin un appendice contient les rudiments sur les fonctions de Jacobi nécessaires à une compréhension complète de notre travail. Néanmoins sa lecture n'est pas indispensable et le rôle des fonctions de Jacobi dans notre travail est de permettre une représentation globale de la sphère de petit rayon dans le cas intégrable. Un point intéressant de cet appendice est de donner heuristiquement une interprétation de la propriété de rigidité dans le cas intégrable comme le flambage d'une poutre en théorie de l'élasticité. Enfin cet article contient une abondante bibliographie dont le rôle précis est discuté dans chaque paragraphe. 


\subsubsection{Précision}

Tout résultat utilisant une simulation numérique non triviale est donné à titre de conjecture.

Ce travail n'aurait pas abouti sans l'aide de I. Kupka, E. Trélat et bien sûr Mathematica.

\section{CAlCuls et PROPRIÉtÉS PRÉliminaIRES - INTERPRÉtATION GÉOMÉTRIQUE DES INVARIANTS}

\subsection{Forme normale graduée - Cas isopérimétrique}

Notre analyse est localisée dans un voisinage $U$ de 0 . La forme normale calculée dans notre article [1] pour le problème SR dans le cas Martinet est donnée par le couple $(D, g)$ où $D=\operatorname{ker} \omega, \omega=d z-\frac{y^{2}}{2} d x$ et la métrique $g$ est une somme de carrés $a d x^{2}+c d y^{2}$ où $a$ et $c$ sont des germes de fonctions analytiques en 0 de la forme $a=1+y F(q), c=1+G(q)$, avec $G_{\mid x=y=0}=0$ et $q=(x, y, z)$ sont les coordonnées de $\mathbf{R}^{3}$. Dans cette forme la distribution est entièrement normalisée et les géodésiques anormales sont contenues dans la surface de Martinet, lieu des points où $\omega$ n'est pas une forme de contact, identifiée à $y=0$ et sont les droites $z=z_{0}$. En paramétrisant les courbes par la longueur d'arc la géodésique anormale est donnée par $a: t \rightarrow( \pm t, 0,0)$. Notre normalisation n'utilise qu'un sous-groupe du groupe de jauge et une forme normale plus réduite a été calculée dans [2]. Cette forme repose aussi sur un principe géométrique différent car sont normalisées les géodésiques normales transverses à la surface de Martinet. Dans notre étude locale les coordonnées $(x, y, z)$ sont graduées en appliquant la règle expliquée dans [20] : le poids des variables $x, y$ est 1 et le poids de $z$ est 3 . On a la graduation suivante pour les champs de vecteurs : le poids de $\frac{\partial}{\partial x}$ et de $\frac{\partial}{\partial y}$ est -1 et le poids de $\frac{\partial}{\partial z}$ est -3 . On introduit les champs de vecteurs suivants :

$$
G_{1}=\frac{\partial}{\partial x}+\frac{y^{2}}{2} \frac{\partial}{\partial z}, \quad G_{2}=\frac{\partial}{\partial y}
$$

qui engendrent la distribution $D$. Notons $($,$) le produit scalaire associé à g$, on a donc :

$$
\left(G_{1}, G_{1}\right)=a, \quad\left(G_{1}, G_{2}\right)=0,\left(G_{2}, G_{2}\right)=c
$$

Deux champs de vecteurs orthonormés engendrant $D$ sont obtenus en posant

$$
F_{1}=\frac{1}{\sqrt{a}} G_{1}, \quad F_{2}=\frac{1}{\sqrt{c}} G_{2} .
$$

On peut définir sur $U$ une métrique riemannienne dont la restriction à $D$ forme la métrique originale en complétant $F_{1}, F_{2}$ par $F_{3}=\frac{\partial}{\partial z}$ et en décrétant que $F_{1}, F_{2}, F_{3}$ forment un repère orthonormé.

La forme normale d'ordre $p$ où $p$ est un entier relatif vérifiant $p \geq 1$ est définie en ne prenant que les termes $F_{1}^{p}, F_{2}^{p}$ d'ordre $\leq p$ dans la série de Taylor de $F_{1}, F_{2}$. Observons qu'à tout ordre $F_{1}^{p}, F_{2}^{p}$ engendrent $D$. En identifiant par convention deux éléments dont les formes normales coincident à l'ordre $p$ on obtient les deux représentants suivants pour les formes normales d'ordre -1 et 0 :

- Forme normale d'ordre -1:

$$
g=d x^{2}+d y^{2}: \text { cas plat }
$$

- Forme normale d'ordre 0:

$$
g=(1+\alpha y)^{2} d x^{2}+(1+\beta x+\gamma y)^{2} d y^{2}, \alpha, \beta, \gamma \in \mathbf{R}
$$


La forme normale d'ordre -1 est identique à celle calculée dans [2] et celle d'ordre 0 contient le même nombre de paramètres. On peut donc utiliser l'une ou l'autre et on sera de toute façon amené ultérieurement à définir une forme normale unique pour étudier les géodésiques.

Le problème est dit isopérimétrique quand les fonctions $a$ et $c$ de $g$ ne dépendent pas de $z$. On note alors $g_{R}$ la métrique riemannienne induite par $g$ sur le plan $(x, y) \sim \mathbf{R}^{3} / \frac{\partial}{\partial z}$. Par convention dans le cas isopérimétrique on se restreint à des difféomorphismes de jauge $\varphi:(x, y, z) \rightarrow(X, Y, Z)$ tels que $X_{z}=Y_{z}=0$, voir [1] et la métrique induite $g_{R}$ est un covariant du problème. Dans cet article on restreint notre étude à la forme normale d'ordre 0 qui est de fait isopérimétrique.

\subsection{Géodésiques (cas isopérimétrique)}

Le problème de minimiser l'énergie qui équivaut au problème de minimiser la longueur. Il s'écrit comme le problème de contrôle optimal :

$$
\frac{d q(t)}{d t}=\sum_{i=1}^{2} u_{i}(t) G_{i}(q(t)) \min _{u(.)} \int_{0}^{T}\left(a(q(t)) u_{1}^{2}(t)+c(q(t)) u_{2}^{2}(t)\right) d t
$$

et le pseudo-hamiltonien associé aux géodésiques normales est :

$$
H_{\frac{1}{2}}(q, p, u)=\sum_{i=1}^{2} u_{i}\left\langle p, G_{i}(q)\right\rangle-\frac{1}{2}\left(a u_{1}^{2}+c u_{2}^{2}\right)
$$

où $p=\left(p_{x}, p_{y}, p_{z}\right)$. Le contrôle associé à une géodésique se calcule en résolvant l'équation $\frac{\partial H_{\frac{1}{2}}}{\partial u}=0$ et l'on obtient

$$
u_{1}=\frac{1}{a}\left(p_{x}+p_{z} \frac{y^{2}}{2}\right), \quad u_{2}=\frac{p_{y}}{c}
$$

et le hamiltonien associé est :

$$
H_{n}(q, p)=\frac{1}{2}\left(u_{1}^{2} a+u_{2}^{2} c\right)=\frac{1}{2}\left[\frac{\left(p_{x}+p_{z} \frac{y^{2}}{2}\right)^{2}}{a}+\frac{p_{y}^{2}}{c}\right] .
$$

Hypothèse 2.1. Dans la suite de cet article on va supposer que nous sommes dans la situation isopérimétrique et donc que $H_{n}$ ne dépend pas de $z$. Dans ce cas $z$ est une coordonnée cyclique et $p_{z}$ est une intégrale première.

Les relèvements géodésiques normaux vérifient les équations suivantes :

$$
\begin{aligned}
\dot{x} & =\frac{1}{a}\left(p_{x}+p_{z} \frac{y^{2}}{2}\right) \\
\dot{y} & =\frac{p_{y}}{c} \\
\dot{z} & =\frac{y^{2}}{2 a}\left(p_{x}+p_{z} \frac{y^{2}}{2}\right) \\
\dot{p_{x}} & =\frac{p_{y}^{2} c_{x}}{2 c^{2}}+\frac{\left(p_{x}+p_{z} \frac{y^{2}}{2}\right)^{2}}{2 a^{2}} a_{x} \\
\dot{p_{y}} & =\frac{p_{y}^{2} c_{y}}{2 c^{2}}+\frac{\left(p_{x}+p_{z} \frac{y^{2}}{2}\right)^{2}}{2 a^{2}} a_{y}-\frac{p_{x}+p_{z} \frac{y^{2}}{2}}{a} p_{z} y \\
\dot{p_{z}} & =0
\end{aligned}
$$


Une autre représentation de ces équations est obtenue en introduisant le vecteur $P=\left(P_{1}, P_{2}, P_{3}\right)$ de $\mathbf{R}^{3}$ où $P_{i}=\left\langle p, F_{i}(q)\right\rangle$. Le hamiltonien prend alors la forme simple :

$$
H_{n}=\frac{1}{2}\left(P_{1}^{2}+P_{2}^{2}\right)
$$

où $P_{1}=\frac{p_{x}+p_{z} \frac{y^{2}}{2}}{\sqrt{a}}, P_{2}=\frac{p_{y}}{\sqrt{c}}, P_{3}=p_{z}$ et les géodésiques normales vérifient les équations $(1.5)$ dans les coordonnées $(q, P)$ qui peuvent être développées en utilisant les séries : $a=(1+\alpha y)^{2}+\ldots, c=(1+\beta x+\gamma y)^{2}+\ldots$.

Les propriétés suivantes se déduisent immédiatement des équations.

Lemme 2.2. Si $a_{x}=c_{x}=0$, alors $x$ est une coordonnée cyclique et les équations dont les solutions sont les géodésiques normales s'intègrent par quadratures.

Lemme 2.3. La géodésique anormale $a: t \mapsto( \pm t, 0,0)$ n'est pas strictement anormale si et seulement si la restriction de $a_{y}$ au plan de Martinet $y=0$ est nulle. Dans ce cas cette géodésique est la projection d'un relèvement géodésique normal où le choix de $p_{z}$ est arbitraire. En particulier elle peut être immergée dans le flot où $p_{z}=0$. D'autre part toute la ligne $y=z=0$ privée de 0 est formée de points conjugués.

\subsection{Définition du feuilletage $(\mathcal{F})$ pour la forme normale graduée d'ordre 0}

En utilisant pour $\theta \neq k \pi$ les coordonnées cylindrique : $P_{1}=\cos \theta, P_{2}=\sin \theta, P_{3}=p_{z}=\lambda$ les équations géodésiques s'écrivent

$$
\begin{aligned}
& \dot{x}=\frac{\cos \theta}{\sqrt{a}} \\
& \dot{y}=\frac{\sin \theta}{\sqrt{c}} \\
& \dot{z}=\frac{y^{2}}{2} \frac{\cos \theta}{\sqrt{a}} \\
& \dot{\theta}=-\frac{1}{\sqrt{a} \sqrt{c}}\left[y \lambda-\frac{a_{y}}{2 \sqrt{a}} \cos \theta+\frac{c_{x}}{2 \sqrt{c}} \sin \theta\right]
\end{aligned}
$$

et en utilisant la forme normale graduée d'ordre 0 où $a=(1+\alpha y)^{2}, c=(1+\beta x+\gamma y)^{2}$, la dernière équation se simplifie en :

$$
\dot{\theta}=-\frac{1}{\sqrt{a} \sqrt{c}}(y \lambda-\alpha \cos \theta+\beta \sin \theta) .
$$

Ces équations définissent un feuilletage $(\tilde{\mathcal{F}})$ de codimension un dans le plan $(y, \theta)$. En effet en utilisant la paramétrisation : $\sqrt{a} \sqrt{c} \frac{d}{d t}=\frac{d}{d \tau}$ et en notant ' la dérivée par rapport à $\tau$ les équations se projettent en :

$$
\begin{aligned}
& y^{\prime}=\sin \theta(1+\alpha y) \\
& \theta^{\prime}=-(y \lambda-\alpha \cos \theta+\beta \sin \theta) .
\end{aligned}
$$

En dérivant le seconde équation et en réduisant on obtient :

$$
\theta^{\prime \prime}+\lambda \sin \theta+\alpha^{2} \sin \theta \cos \theta-\alpha \beta \sin ^{2} \theta+\beta \cos \theta \theta^{\prime}=0 .
$$

Pour $\lambda \neq 0$, on pose $s=\tau \sqrt{|\lambda|}$ et l'on obtient l'équation

$$
\frac{d^{2} \theta}{d s^{2}}+\frac{\lambda}{|\lambda|} \sin \theta+\frac{\alpha}{\operatorname{mid\lambda |}} \sin \theta(\alpha \cos \theta-\beta \sin \theta)+\frac{\beta}{\sqrt{|\lambda|}} \cos \theta \frac{d \theta}{d s}=0 .
$$




\subsubsection{Notation}

On note $(\mathcal{F})$ le feuilletage défini par l'équation $(2.5)$ sur le cylindre $\left(\mathrm{e}^{i \theta}, \theta^{\prime}\right)$ qui coincide pour $\lambda \neq 0$ avec le feuilletage défini par (2.6).

\subsection{Aspect asymptotique}

On se propose d'utiliser le feuilletage $(\mathcal{F})$ comme un substitut à une compactification pour étudier le comportement des géodésiques normales minimisantes de petite longueur $r$ au voisinage de la direction anormale. D'après le paragraphe 1.3.3, on a dans le cas plat la relation $r=\frac{2}{\sqrt{|\lambda|}} K(k)$ où $k^{2}=\frac{1-\cos \theta(0)}{2}$ et au voisinage de la direction anormale on a $\theta(0) \rightarrow n \pi$ et $k \rightarrow 0,1$. Par ailleurs pour $k \rightarrow 0, K(k) \sim \frac{\pi}{2}$ et pour $k \rightarrow 1$, $K(k) \sim-\log \sqrt{1-k^{2}}$. Donc pour $r$ petit, $\lambda$ est grand. Par ailleurs les paramètres $\alpha, \beta, \gamma$ de la métrique sont eux fixés. On définit le feuilletage asymptotique $\left(\mathcal{F}_{a}\right)$ en supposant $\lambda \gg \alpha, \beta, \gamma$ dans l'équation (2.6). En introduisant alors le petit paramètre : $\varepsilon=\frac{1}{\sqrt{|\lambda|}}$, il est donné par l'équation

$$
\frac{d^{2} \theta}{d s^{2}}+\frac{\lambda}{|\lambda|} \sin \theta+\varepsilon \beta \cos \theta \frac{d \theta}{d s}+\varepsilon^{2} \alpha \sin \theta(\alpha \cos \theta-\beta \sin \theta)=0 \text {. }
$$

\subsubsection{Quelques propriétés du feuilletage asymptotique}

On peut observer que dans notre étude on peut supposer $\lambda>0$ car en changeant $\lambda$ en $-\lambda$ on permute simplement la nature des points singuliers.

Cas plat. Le feuilletage est défini par $\frac{d^{2} \theta}{d s^{2}}+\sin \theta=0$. C'est un pendule qui possède une intégrale première globale analytique. Il a deux positions d'équilibre, l'une donnée par $\theta=\theta^{\prime}=0$ est un centre et l'autre donnée par $\theta=\pi$ et $\theta^{\prime}=0$ est un col. Sur le cylindre toutes les trajectoires sont périodiques sauf les deux séparatrices $\Sigma_{1}$ et $\Sigma_{2}$ qui ont la propriété importante d'être des connections de col.

\subsubsection{Le cas $\beta \neq 0$}

Dans ce cas comme $\varepsilon$ est un petit paramètre les propriétés stables de $\left(\mathcal{F}_{a}\right)$ sont décrites par l'équation :

$$
\frac{d^{2} \theta}{d s^{2}}+\sin \theta+\varepsilon \beta \cos \theta \frac{d \theta}{d s}=0
$$

Cette équation est une équation fondamentale de la géométrie $S R$ et est analysée en détail dans le paragraphe 5. Une propriété importante pour la signification géométrique des invariants est la suivante.

Lemme 2.4. Pour $\beta \neq 0$, l'équation (2.8) n'admet pas d'intégrale première globale continue.

Preuve. La preuve est simple. Pour $\beta \neq 0$, l'origine devient un foyer qui n'admet pas localement une intégrale première continue.

\subsubsection{Le cas $\beta=0$}

Dans ce cas le feuilletage $\left(\mathcal{F}_{a}\right)$ est décrit par l'équation

$$
\frac{d^{2} \theta}{d s^{2}}+\sin \theta\left(1+\varepsilon^{2} \alpha^{2} \cos \theta\right)=0
$$

Une telle équation admet une interprétation importante en théorie de l'élasticité qui est expliquée dans l'appendice. Elle a deux propriétés remarquables. La première est que le flot admet la symétrie $\left(\theta, \theta^{\prime}\right) \rightarrow\left(-\theta,-\theta^{\prime}\right)$. La seconde justifie un lemme.

Lemme 2.5. L'équation (2.9) est intégrable (en termes d'intégrales elliptiques). 


\subsection{Quasi-homogénéité et développements formels}

Pour obtenir un développement formel de l'application exponentielle au voisinage de 0 on éclate le système (1.5) en utilisant les poids du modèle plat. En effet en posant :

$$
\begin{gathered}
x=\varepsilon X, y=\varepsilon Y, z=\varepsilon^{3} Z \\
P_{1}=Q_{1}, P_{2}=Q_{2}, P_{3}=\frac{Q_{3}}{\varepsilon^{2}}
\end{gathered}
$$

et en paramétrisant les trajectoires par $d s=\frac{d t}{\varepsilon}$, les équations (1.5) s'écrivent

$$
\begin{aligned}
\frac{d X}{d s} & =\frac{1}{\sqrt{a}} Q_{1} \\
\frac{d Y}{d s} & =\frac{1}{\sqrt{c}} Q_{2} \\
\frac{d Z}{d s} & =\frac{Y^{2}}{2 \sqrt{a}} Q_{1} \\
\frac{d Q_{1}}{d s} & =\frac{Q_{2}}{\sqrt{a} \sqrt{c}}\left[Y Q_{3}-\frac{\varepsilon a_{y}}{2 \sqrt{a}} Q_{1}+\frac{\varepsilon c_{x}}{2 \sqrt{c}} Q_{2}\right] \\
\frac{d Q_{2}}{d s} & =-\frac{Q_{1}}{\sqrt{a} \sqrt{c}}\left[Y Q_{3}-\frac{\varepsilon a_{y}}{2 \sqrt{a}} Q_{1}+\frac{\varepsilon c_{x}}{2 \sqrt{c}} Q_{2}\right] \\
\frac{d Q_{3}}{d s} & =0 .
\end{aligned}
$$

Introduisons l'angle $\theta$ en posant $Q_{1}=\cos \theta, Q_{2}=\sin \theta$, on obtient les équations :

$$
\begin{aligned}
\frac{d X}{d s} & =\frac{1}{\sqrt{a}} \cos \theta \\
\frac{d Y}{d s} & =\frac{1}{\sqrt{c}} \sin \theta \\
\frac{d Z}{d s} & =\frac{Y^{2}}{2 \sqrt{a}} \cos \theta \\
\frac{d \theta}{d s} & =-\frac{1}{\sqrt{a} \sqrt{c}}\left[Y Q_{3}-\frac{\varepsilon a_{y}}{2 \sqrt{a}} \cos \theta+\frac{\varepsilon c_{x}}{2 \sqrt{c}} \sin \theta\right] .
\end{aligned}
$$

On doit intégrer ces équations avec $P_{3}=\lambda=\frac{Q_{3}}{\varepsilon^{2}}$ et l'on doit considérer les cas $Q_{3}= \pm 1$ et $Q_{3}=0$. On pose :

$$
\begin{aligned}
X & =X_{0}+\varepsilon X_{1}+o(\varepsilon) \\
Y & =Y_{0}+\varepsilon Y_{1}+o(\varepsilon) \\
Z & =Z_{0}+\varepsilon Z_{1}+o(\varepsilon) \\
\theta & =\theta_{0}+\varepsilon \theta_{1}+o(\varepsilon)
\end{aligned}
$$

et on impose les conditions initiales :

$$
\begin{aligned}
X_{i}(0) & =Y_{i}(0)=Z_{i}(0)=0 \\
\theta_{0}(0) & =\theta(0), \quad \theta_{i}(0)=0
\end{aligned}
$$


pour $i \geq 1$. En utilisant alors le développement gradué : $a(x, y)=1+\bar{\alpha} y+\ldots, c(x, y)=1+\bar{\beta} x+\bar{\gamma} y+\ldots$, on obtient alors les équations suivantes.

2.5.1. Cas $Q_{3}=1$ (le cas $Q_{3}=-1$ est semblable)

On a :

$$
\begin{aligned}
\frac{d X_{0}}{d s} & =\cos \theta_{0} \\
\frac{d Y_{0}}{d s} & =\sin \theta_{0} \\
\frac{d Z_{0}}{d s} & =\frac{Y_{0}^{2}}{2} \sin \theta_{0} \\
\frac{d \theta_{0}}{d s} & =-Y_{0} .
\end{aligned}
$$

Ce système correspond au cas plat, l'angle $\theta_{0}$ étant une solution oscillante $\left(\frac{d \theta}{d s}(0)=0\right)$ du pendule :

$$
\frac{d^{2} \theta_{0}}{d s^{2}}+\sin \theta_{0}=0
$$

Par ailleurs :

$$
\begin{aligned}
& \frac{d X_{1}}{d s}=-\frac{\bar{\alpha} Y_{0}}{2} \cos \theta_{0}-\theta_{1} \sin \theta_{0} \\
& \frac{d Y_{1}}{d s}=-\frac{\bar{\beta} X_{0}+\bar{\gamma} Y_{0}}{2} \sin \theta_{0}+\theta_{1} \cos \theta_{0} \\
& \frac{d Z_{1}}{d s}=Y_{0} Y_{1} \cos \theta_{0}-\frac{\bar{\alpha} Y_{0}^{3}}{4} \cos \theta_{0}-\frac{Y_{0}^{2}}{2} \theta_{1} \sin \theta_{0} \\
& \frac{d \theta_{1}}{d s}=-Y_{1}+\frac{1}{2}\left(\bar{\alpha} \cos \theta_{0}-\bar{\beta} \sin \theta_{0}\right)+\frac{Y_{0}}{2}\left[(\bar{\alpha}+\bar{\gamma}) Y_{0}+\bar{\beta} X_{0}\right] .
\end{aligned}
$$

La propriété principale du système (2.15) est la suivante. On observe que $\theta_{1}$ est solution d'une équation du type :

$$
\frac{d^{2} \theta_{1}}{d s^{2}}+\theta_{1} \cos \theta_{0}=K\left(X_{0}, Y_{0}, Z_{0}, \theta_{0}\right)
$$

où $\theta_{0}$ est une fonction périodique. C'est une équation de type Mathieu avec un terme forçant. Cette équation correspond à l'équation aux variations de notre problème et mesure la stabilité du cas plat.

Ce calcul permet d'obtenir un développement formel pour l'application exponentielle. En effet de $P_{3}=\lambda=\frac{Q_{3}}{\varepsilon^{2}}$ et $Q_{3}=1$ on tire $\varepsilon=\frac{1}{\sqrt{\lambda}}$. Les composantes de l'application exponentielle vérifient donc :

$$
\begin{aligned}
& x(t, \lambda, \theta(0))=\frac{1}{\sqrt{\lambda}} X_{0}(t \sqrt{\lambda}, 1, \theta(0))+\frac{1}{\lambda} X_{1}(t \sqrt{\lambda}, 1, \theta(0))+\ldots \\
& y(t, \lambda, \theta(0))=\frac{1}{\sqrt{\lambda}} Y_{0}(t \sqrt{\lambda}, 1, \theta(0))+\frac{1}{\lambda} Y_{1}(t \sqrt{\lambda}, 1, \theta(0))+\ldots \\
& z(t, \lambda, \theta(0))=\frac{1}{\sqrt{\lambda}} Z_{0}(t \sqrt{\lambda}, 1, \theta(0))+\frac{1}{\lambda} Z_{1}(t \sqrt{\lambda}, 1, \theta(0))+\ldots
\end{aligned}
$$

où $X_{0}, Y_{0}, Z_{0}$ sont les solutions du cas plat et $X_{1}, Y_{1}, Z_{1}$ sont solutions des équations (2.15). On observe que les courbes sont paramétrisées par $s=t \sqrt{\lambda}$. Le problème de stabilité est de contrôler le terme de perturbation $X_{1}, Y_{1}, Z_{1}$, par exemple dans le calcul de la sphère. 


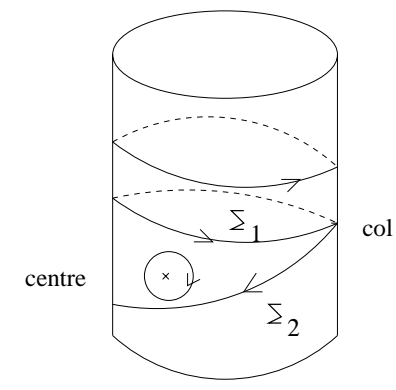

FiguRE 4

\subsubsection{Le problème de stabilité}

L'étude du cas plat montre que la partie intéressante de l'étude du problème de Martinet est la nature de la sphère et du front d'onde au voisinage de la direction anormale : $\theta(0) \rightarrow \pi$ où l'application retour n'est pas propre et $s=t \sqrt{\lambda}, t=r$ tend vers l'infini. Géométriquement pour le feuilletage $(\mathcal{F})$ on est concerné par les calculs des solutions au voisinage d'une séparatrice. Cela pose deux problèmes.

1. Problème 1 : problème topologique. Pour le problème plat, au voisinage de la séparatrice $\Sigma_{1}$, le feuilletage $(\mathcal{F})$ sur le cylindre possède des trajectoires fermées homotopes à 0 et des trajectoires fermées non homotopes $\grave{a} 0$, voir figure 4 . Ce phénomène est source d'instabilité et l'on discute ce problème dans le paragraphe 4 : c'est le phénomène de doublement de période.

2. Problème 2. Un autre problème de stabilité se greffe au problème précédent. En évaluant les solutions sur un temps $s=t \sqrt{\lambda} \rightarrow+\infty$ il y a d'autres effets séculaires qui ne sont pas pris en compte dans les développements précédents. En effet dans $\theta_{0}$ on ne considère que des trajectoires périodiques dont l'amplitude et la phase sont constantes. Un meilleur développement est obtenu en utilisant la méthode dite de Krylov-Bogoliubov inspirée de Van der Pol (voir [6]) et qui consiste à développer $\theta$ en $\theta_{0}(\varepsilon)+\varepsilon \theta_{1}(\varepsilon)+o(\varepsilon)$ où l'amplitude et la phase de $\theta_{0}(\varepsilon)$ varient lentement. Ce type de méthode et le calcul chronologique peuvent être utilisées dans le cas non intégrable. Un autre point de vue similaire à celui utilisé pour analyser le seizième problème de Hilbert sur les cycles limites est de considérer le cas général comme une perturbation du cas intégrable. Notre article est dans cet esprit.

\subsubsection{Le cas $Q_{3}=0$}

Dans ce cas la situation est simple. La projection des géodésiques normales dans le plan $(x, y)$ sont les géodésiques de la métrique riemannienne induite :

$$
g_{R}=a(x, y) d x^{2}+c(x, y) d y^{2}
$$

et l'on a :

$$
\begin{aligned}
\dot{X}_{0} & =\cos \theta_{0} \\
\dot{Y}_{0} & =\sin \theta_{0} \\
\dot{\theta_{0}} & =0
\end{aligned}
$$


et les trajectoires dans le plan $(x, y)$ sont des droites. Le terme de perturbation est donné par :

$$
\begin{aligned}
& \dot{X}_{1}=-\frac{\bar{\alpha} Y_{0}}{2} \cos \theta_{0}-\theta_{1} \sin \theta_{0} \\
& \dot{Y}_{1}=-\frac{\bar{\beta} X_{0}+\bar{\gamma} Y_{0}}{2} \sin \theta_{0}+\theta_{1} \cos \theta_{0} \\
& \dot{\theta_{1}}=\frac{1}{2}\left(\bar{\alpha} \cos \theta_{0}-\bar{\beta} \sin \theta_{0}\right) .
\end{aligned}
$$

En tronquant $a(x, y)=1+\bar{\alpha} y, c(x, y)=1+\bar{\beta} x+\bar{\gamma} y$ à l'ordre 0 la courbure de Gauss de la métrique est la suivante :

$$
K=\frac{R_{1212}}{(1+\bar{\alpha} y)(1+\bar{\beta} x+\bar{\gamma} y)}
$$

où

$$
R_{1212}=\frac{\bar{\alpha}^{2}}{4(1+\bar{\alpha} y)}+\frac{\bar{\beta}^{2}+\bar{\alpha} \bar{\gamma}}{4(1+\bar{\beta} x+\bar{\gamma} y)}
$$

En particulier on observe que la courbure $K$ est nulle pour tout réel $\bar{\gamma}$ si $\bar{\alpha}=\bar{\beta}=0$.

\subsection{Le cas intégrable}

\subsubsection{Paramétrisation et propriétés}

Si la métrique $g$ est de la forme $a(y) d x^{2}+c(y) d y^{2}$, les équations dont les solutions sont les relèvements géodésiques normaux sont intégrables par quadratures. Cette propriété permet de calculer l'application exponentielle en particulier au voisinage du plan $y=0$. On utilise les formules suivantes.

En utilisant (1.5), l'équation dite équation caractéristique :

$$
H_{n}=\frac{1}{2}\left(P_{1}^{2}+P_{2}^{2}\right)=\frac{1}{2}
$$

avec $P_{1}(y)=\frac{1}{\sqrt{a}}\left(p_{x}+p_{z} \frac{y^{2}}{2}\right), P_{2}(y)=\frac{1}{\sqrt{c}} p_{y}$ où $p_{x}$ et $p_{z}$ sont des intégrales premières s'écrit :

$$
(\sqrt{c} \dot{y})^{2}+\left(\frac{p_{x}+p_{z} \frac{y^{2}}{2}}{\sqrt{a}}\right)^{2}=1 .
$$

En utilisant le temps $d \tau=\frac{d t}{\sqrt{c}}$ cette équation décrit l'évolution d'un système mécanique où $y$ est la position d'une particule dans un champ de potentiel : $V(y)=P_{1}^{2}(y)$ et d'énergie donnée.

Notons $e()=.(x(),. y(),. z()$.$) une géodésique normale définie sur [0, T]$, paramétrisée par la longueur d'arc et issue de 0 . Si $y($.$) n'est pas identiquement nul on note 0<t_{1}<\ldots<t_{n} \leq T$ les temps successifs définis par $y\left(t_{i}\right)=0$. On introduit

$$
\sigma=\left\{\begin{array}{l}
\text { signe } \dot{y}(0) \text { si } \dot{y}(0) \neq 0 \\
\text { signe } \ddot{y}(0) \text { si } \dot{y}(0)=0 .
\end{array}\right.
$$

Faisons maintenant l'hypothèse que le mouvement de $y$ est périodique de période $\mathcal{P}$ et posons :

$$
y_{+}=\max _{t \in[0, \mathcal{P}]} y(t), \quad y_{-}=\min _{t \in[0, \mathcal{P}]} y(t) .
$$


En paramétrisant les géodésiques par $y$ on doit résoudre les équations :

$$
\begin{gathered}
\frac{d x}{d y}=\frac{\sqrt{c}}{\sqrt{a}} \frac{P_{1}}{P_{2}}, \frac{d z}{d y}=\frac{y^{2}}{2} \frac{\sqrt{c}}{\sqrt{a}} \frac{P_{1}}{P_{2}} \\
d t=\frac{\sqrt{c} d y}{P_{2}}
\end{gathered}
$$

où $P_{2}(y)=\sigma \sqrt{1-P_{1}^{2}(y)}$ pour $t \in\left[0, t_{1}\right]$.

Si $y(T)=0, T=t_{N}$, on obtient les formules suivantes :

- $N$ impair

$$
\begin{aligned}
& x(T)=2 \int_{0}^{y_{\sigma}} \frac{\sigma \sqrt{c}}{\sqrt{a}} \frac{P_{1}(y)}{\sqrt{1-P_{1}^{2}(y)}} d y+(N-1) \int_{y_{-}}^{y_{+}} \frac{\sqrt{c}}{\sqrt{a}} \frac{P_{1}(y)}{\sqrt{1-P_{1}^{2}(y)}} d y \\
& z(T)=\int_{0}^{y_{\sigma}} \frac{\sigma \sqrt{c}}{\sqrt{a}} \frac{y^{2} P_{1}(y)}{\sqrt{1-P_{1}^{2}(y)}} d y+(N-1) \int_{y_{-}}^{y_{+}} \frac{\sqrt{c}}{2 \sqrt{a}} \frac{y^{2} P_{1}(y)}{\sqrt{1-P_{1}^{2}(y)}} d y
\end{aligned}
$$

- $N$ pair

$$
\begin{gathered}
x(T)=N \int_{y_{-}}^{y_{+}} \frac{\sqrt{c}}{\sqrt{a}} \frac{P_{1}(y)}{\sqrt{1-P_{1}^{2}(y)}} d y \\
z(T)=N \int_{y_{-}}^{y_{+}} \frac{\sqrt{c}}{2 \sqrt{a}} \frac{y^{2} P_{1}(y)}{\sqrt{1-P_{1}^{2}(y)}} d y
\end{gathered}
$$

et la période est donnée par :

$$
\mathcal{P}=2 \int_{y_{-}}^{y_{+}} \frac{\sqrt{c}}{\sqrt{1-P_{1}^{2}(y)}} d y .
$$

En utilisant ces relations on observe la propriété géométrique suivante. Si $\dot{y}(0) \neq 0$, les deux géodésiques distinctes associées à $\dot{y}(0)=p_{y}(0)$ et $-\dot{y}(0), p_{x}, p_{z}$ étant fixés intersectent avec la même longueur $t_{2 p}$ le plan $y=0$ aux mêmes points $\left(x\left(t_{2 p}\right), z\left(t_{2 p}\right)\right)$. On en déduit donc le résultat suivant.

Proposition 2.6. Considérons le cas intégrable et soit e $(t)=(x(t), y(t), z(t))$ une géodésique normale issue de 0 paramétrisée par la longueur d'arc telle que $y(t)$ soit périodique, $\dot{y}(0) \neq 0$ et associée à $p_{x}, p_{z}$. Soit $\tilde{e}(t)=(\tilde{x}(t)$, $\tilde{y}(t), \tilde{z}(t)), \tilde{e}(0)=0$ la géodésique normale paramétrisée par la longueur d'arc et associée à la condition initiale $-\dot{y}(0), p_{x}$ et $p_{z}$. Alors les deux géodésiques e et $\tilde{e}$ sont distinctes, mais leurs intersections paires avec le plan $y=0$ sont identiques et ont même longueur. En particulier e(.) n'est pas minimisante au delà de sa seconde intersection avec le plan $y=0$.

Preuve. Soit $t_{2}$ le temps correspondant à la deuxième intersection de la géodésique $e($.$) avec y=0$. On déduit du principe du maximum que dans le cas Martinet les courbes minimisantes sont analytiques. La conclusion résulte du lemme 4.13 de [1].

Remarque 2.7. On peut utiliser des formules identiques pour évaluer l'application exponentielle sans la contrainte $y(T)=0$ et en particulier pour les extrémités au voisinage de la surface $y=0$.

\subsubsection{Equation caractéristique sous forme normale}

Dans le cas intégrable l'étude des géodésiques normales repose sur l'analyse et l'intégration de l'équation caractéristique. C'est un exercice classique de mécanique qui se ramène au calcul des racines réelles de l'équation $1-P_{1}^{2}(y)=0$. Clairement une forme normale de notre problème est 
donc de trouver un difféomorphisme local dans $\mathbf{R}$ pour normaliser ces racines. De ce point de vue le rôle des intégrales elliptiques de première espèce dans notre problème est clair. Ce sont précisément des modèles où le potentiel prend une forme canonique. Ce type de simplification est très important pour analyser l'application exponentielle. On va donc décrire cette réduction pour la forme normale graduée d'ordre 0 .

L'équation caractéristique est : $P_{1}^{2}+P_{2}^{2}=1$ où $P_{1}=\frac{p_{x}+p_{z} \frac{y^{2}}{2}}{\sqrt{a}}, P_{2}=\frac{p_{y}}{\sqrt{c}}$ et $\dot{y}=\frac{P_{2}}{\sqrt{c}}, p_{x}, p_{z}$ étant des constantes : $p_{x}=\cos \theta(0)$ et $p_{z}=\lambda$. Par symétrie, on peut supposer $\lambda \geq 0$. On analyse l'équation caractéristique dans le cas $\lambda>0$. On a donc l'équation

$$
(\sqrt{c} \dot{y})^{2}+\left(\frac{p_{x}+p_{z} \frac{y^{2}}{2}}{\sqrt{a}}\right)^{2}=1
$$

que l'on peut écrire avec $\lambda=p_{z}$ :

$$
(\sqrt{a} \sqrt{c} \dot{y})^{2}+\left(p_{x}+p_{z} \frac{y^{2}}{2}\right)^{2}=a
$$

et en introduisant la nouvelle paramétrisation :

$$
d \tau=\frac{d t}{\sqrt{a} \sqrt{c}}
$$

il vient :

$$
\left(\frac{d y}{d \tau}\right)^{2}+\left(p_{x}+p_{z} \frac{y^{2}}{2}\right)^{2}=a
$$

où $a=(1+\alpha y)^{2}$ dans la forme normale graduée d'ordre 0 . On a donc :

$$
\left(\frac{d y}{d \tau}\right)^{2}=F(y)
$$

où $F(y)=(1+\alpha y)^{2}-\left(p_{x}+p_{z} \frac{y^{2}}{2}\right)^{2}$ et l'étude repose sur les racines de l'équation $F(y)=0$. On observe que $F$ est une quartique qui se factorise en $F=F_{1} F_{2}$ où :

$$
F_{1}=(1+\alpha y)-\left(p_{x}+\lambda \frac{y^{2}}{2}\right), F_{2}=(1+\alpha y)+\left(p_{x}+\lambda \frac{y^{2}}{2}\right)
$$

que l'on peut écrire :

$$
F(y)=\left(2 m^{2}-\frac{\lambda}{2}\left(y-\frac{\alpha}{\lambda}\right)^{2}\right)\left(2 m^{\prime \prime}+\frac{\lambda}{2}\left(y+\frac{\alpha}{\lambda}\right)^{2}\right)
$$

où

$$
2 m^{2}=1-p_{x}+\frac{\alpha^{2}}{2 \lambda}, 2 m^{\prime \prime}=1+p_{x}-\frac{\alpha^{2}}{2 \lambda}
$$

et $m^{2}+m^{\prime \prime}=1, m^{2}>0$ si $\alpha \neq 0$ et $m^{2}>0$ si $\alpha=0$ et $\theta \neq n \pi$. En posant

$$
\eta=\frac{\sqrt{\lambda} y}{2 m}-\frac{\alpha}{2 m \sqrt{\lambda}}, \quad \bar{\eta}=\frac{\sqrt{\lambda} y}{2 m}+\frac{\alpha}{2 m \sqrt{\lambda}}
$$


on peut donc écrire :

$$
F(y)=4 m^{2}\left(1-\eta^{2}\right)\left(m^{\prime \prime}+m^{2} \bar{\eta}^{2}\right)
$$

et $F$ est une quartique dont les racines sont $\eta= \pm 1, \bar{\eta}= \pm \frac{\sqrt{-m^{\prime \prime}}}{m} \in \mathbf{C}$. La situation est dite critique lorsque $m^{\prime \prime}=0$ et $F$ admet une racine double. L'examen de $m^{\prime \prime}=0$ montre la propriété suivante.

Lemme 2.8. Lorsque $\alpha \neq 0$ dans la forme normale graduée d'ordre 0, il existe toujours dans le cas intégrable des géodésiques issues de 0 dites critiques où $F$ admet $\bar{\eta}=0$ comme racine double.

Interprétation géométrique. Dans le cas plat $m^{\prime \prime}=k^{\prime 2}=\sqrt{1-k^{2}}$ où $k^{\prime}$ est le complémentaire du module $k$ des $\overline{\text { fonctions elliptiques. Lorsque }} p_{x} \rightarrow-1, k^{\prime} \rightarrow 0$ et $y$ se comporte comme une sécante hyperbolique, voir [21] p. 39. Dans la projection des géodésiques dans le plan de phase $(\theta, \dot{\theta})$ du pendule, elles tendent vers les séparatrices joignant les points cols. Lorsque $\alpha \neq 0$, ces séparatrices sont des projections de géodésiques issues de 0 . Le rôle géométrique du paramètre $\alpha$ est le suivant. Pour $\alpha \neq 0$, les séparatrices et trajectoires en rotation du pendule correspondent à des projections des géodésiques admissibles en 0.

Forme normale. On peut mettre l'équation caractéristique sous forme normale en utilisant la technique de [21] p. 55. On procède ainsi : $F$ se factorise en $F_{1} F_{2}$ et on considère le faisceau $F_{1}+\nu F_{2}$ des deux formes quadratiques. Si $\alpha \neq 0$, il existe deux réels $\nu_{1}, \nu_{2}$ distincts tels que $F_{1}+\nu_{i} F_{2}$ soient des carrés parfaits notés respectivement : $K_{1}(y-p)^{2}$ et $K_{2}(y-q)^{2}$. En utilisant la transformation homographique

$$
u=(y-p)(y-q)^{-1}
$$

on ramène l'équation caractéristique sous forme normale :

$$
\frac{d y}{\sqrt{F(y)}}=\frac{(p-q)^{-1} d u}{\sqrt{\left(A_{1} u^{2}+B_{1}\right)\left(A_{2} u^{2}+B_{2}\right)}}
$$

et la trajectoire $u(t)$ se calcule à l'aide d'intégrales elliptiques de première espèce.

Hormis le cas critique où $m^{\prime \prime}=0$, il y a deux types de solutions : si la quartique admet 2 racines réelles $u$ se paramétrise à l'aide de la fonction cn et si la quartique admet 4 racines réelles $u$ se paramétrise avec la fonction dn.

Dans le cas où $\alpha=0$, cette étude se simplifie et $F(y)$ est déjà sous la forme normale : $4 k^{2}\left(1-\eta^{2}\right)\left(k^{\prime 2}+k^{2} \eta^{2}\right)$ ce qui conduit directement à une paramétrisation de $\eta$ avec une fonction cn, y étant lié à $\eta$ par une simple homothétie. On déduit de cette étude que l'on peut toujours ramener le feuilletage $(\mathcal{F})$ à un pendule $: \ddot{\theta}+\sin \theta=0$ dans le cas intégrable d'ordre 0 .

Proposition 2.9. Il existe toujours un système de coordonnées sur le cotangent $T^{*} U$ tel que dans le cas intégrable d'ordre $0: g=(1+\alpha y)^{2} d x^{2}+(1+\gamma y)^{2} d y^{2}$, l'oscillation d'une géodésique par rapport au plan de Martinet soit représentée par la dérivée $\dot{\theta}$ des solutions d'un pendule $\ddot{\theta}+\sin \theta=0$. Si $\alpha=0$, on paramétrise les géodésiques issues de 0 à l'aide des solutions oscillantes et si $\alpha \neq 0$ on doit utiliser les trajectoires oscillantes et les trajectoires en rotation.

Une autre conséquence importante de notre réduction est la suivant :

Proposition 2.10. Dans le cas intégrable d'ordre 0 une géodésique possède la propriété $(\mathcal{R})$ de rigidité géodésique.

Preuve. La preuve de la rigidité géodésique est contenue dans [19] pour $\gamma=0$, voir aussi [1]. Dans le cas général on se ramène à cette situation en reparamétrisant. 


\subsubsection{Conclusion}

Notre étude donne déjà une interprétation fine du rôle géométrique des paramètres $\alpha, \beta$, le paramètre $\gamma$ étant absorbé par une reparamétrisation. Elle montre clairement qu'il faut distinguer le cas intégrable $\beta=0$ du cas non intégrable $\beta \neq 0$. Dans le cas intégrable on constate que modulo une certaine complexité de calculs où interviennent des intégrales elliptiques de première et de seconde espèce on peut calculer la sphère et le front d'onde. Cela sera réalisé partiellement dans les deux prochains paragraphes. Par contre le cas non intégrable présente des difficultés techniques d'un autre ordre.

\section{AnAlyse DU CAS $g=d x^{2}+(1+\gamma y)^{2} d y^{2}$}

\subsection{Préliminaires}

L'objectif de ce paragraphe est d'analyser le cas où dans la forme normale graduée d'ordre $0, \alpha=\beta=0$. Cette situation est une déformation à un paramètre du cas plat possédant les propriétés suivantes :

- La courbure de Gauss de la métrique projetée $g_{R}$ est nulle et c'est donc une métrique plate.

- La géodésique anormale $a: t \mapsto( \pm t, 0,0)$ n'est pas strictement anormale.

- L'équation des géodésiques normales est intégrable par quadratures.

On va montrer que l'on peut généraliser l'analyse du cas plat à cette déformation à un paramètre car le rôle du paramètre $\gamma$ est peu sensible géométriquement étant donné qu'il peut être absorbé essentiellement par un changement de paramétrage. L'étude est par ailleurs typique du traitement d'un cas intégrable. On paramétrise l'ensemble des géodésiques, ici avec des fonctions de Jacobi. À l'aide de ce calcul on évalue ensuite la singularité de la trace de la sphère avec le plan $y=0$ et dans la direction anormale, en utilisant des développements asymptotiques convergents. Puis on calcule la trace complète en utilisant la paramétrisation globale des géodésiques.

\subsection{Notations et symétries}

Pour calculer l'ensemble des géodésiques normales paramétrisées par la longueur d'arc et issues de 0 on introduit les paramètres suivants : $P_{1}(0)=p_{x}=\sin \varphi, P_{2}(0)=p_{y}(0)=\cos \varphi\left(\right.$ c'est à dire $\left.\varphi+\theta(0)=\frac{\pi}{2}\right)$, $P_{3}=p_{z}=\lambda$ et l'application exponentielle est définie sur le cylindre : $(\lambda, \varphi, t)$. On observe que lorsque la métrique est de la forme $g=a(y) d x^{2}+c(y) d y^{2}$, les équations géodésiques (2.2) et la métrique sont invariantes pour la transformation : $X=-x, Y=y, Z=-z, P_{X}=-p_{x}, P_{Y}=p_{y}, P_{Z}=-p_{z}$ et l'ensemble des géodésiques paramétrisées par la longueur d'arc sont laissées invariantes par la symétrie $S:(x, y, z) \mapsto(-x, y,-z)$. Donc dans notre étude on peut supposer $\lambda \geq 0$. Une autre symétrie importante est la suivante. En ajoutant aux équations (2.2) l'équation $\dot{\gamma}=0$ on obtient un nouveau système noté (2.2)'. Ce système et la métrique sont laissés invariants par la transformation $\left(x, y, z, p_{x}, p_{y}, p_{z}, \gamma\right) \mapsto\left(x,-y, z, p_{x},-p_{y}, p_{z},-\gamma\right)$. Dans le cas plat : $\gamma=0$ cette propriété implique que la sphère $S(0, r)$ et le front d'onde $W(0, r)$ sont aussi laissés invariants par la symétrie $S^{\prime}:(x, y, z) \mapsto(x,-y, z)$.

\subsection{Géodésiques correspondant à $\lambda=0$}

La métrique $g_{R}$ induite par $g$ est plate. Les géodésiques de cette métrique sont les projections dans le plan $(x, y)$ des géodésiques normales associées à $\lambda=0$. On les calcule aisément. Si $p_{z}=\lambda=0$, on obtient

$$
P_{1}(t)=\sin \varphi, \quad P_{2}(t)=\cos \varphi
$$

et

$$
d x=\tan \varphi \sqrt{c} d y
$$


En utilisant $\sqrt{c}=(1+\gamma y)$, on obtient avec la condition initiale $x(0)=y(0)=0$ :

$$
x=\left(y+\gamma \frac{y^{2}}{2}\right) \tan \varphi
$$

Ce sont des droites dans les coordonnées locales : $X=x, Y=\left(y+\gamma \frac{y^{2}}{2}\right)$. Néanmoins une telle transformation n'est pas induite par un difféomorphisme local laissant ker $\omega$ invariant (ils sont paramétrisés dans [1]) et $\gamma$ est bien un invariant du problème.

Ces droites sont minimisantes pour la métrique riemannienne et correspondent donc a fortiori à des courbes minimisantes $S R$. La géodésique anormale $a: t \mapsto( \pm t, 0,0)$ est associée à la condition initiale $\varphi= \pm \frac{\pi}{2}$ et se projette sur $t \mapsto( \pm t, 0)$. On a donc le résultat suivant.

Lemme 3.1. Les projections des géodésiques associées $\grave{a} \lambda=0$ dans le plan $(x, y)$ sont des droites dans les coordonnées : $X=x, Y=y+\gamma \frac{y^{2}}{2}$ et ces géodésiques sont minimisantes. Parmi ces géodésiques de longueur $r$, seule la géodésique anormale $a: t \mapsto( \pm t, 0,0)$ intersecte la trace de la sphère avec le plan $y=0$ aux points antipodaux : $( \pm r, 0,0)$.

\subsection{Géodésiques normales correspondant à $\lambda>0$}

L'équation caractéristique est donnée par :

$$
\left(\frac{d y}{d \tau}\right)^{2}+P_{1}^{2}(y)=1
$$

où $d \tau=\frac{d t}{\sqrt{c}}, c=(1+\gamma y)^{2}$ et $P_{1}(y)=p_{x}+p_{z} \frac{y^{2}}{2}$ où $p_{x}$ et $p_{z}$ sont des intégrales premières.

Si $y(0)=\dot{y}(0)=0$, la géodésique correspondante est la géodésique anormale $a: t \mapsto( \pm t, 0,0)$. Si $\dot{y}(0) \neq 0$, on introduit $\sigma=\operatorname{sign} \dot{y}(0)$ et l'on peut supposer $\sigma=+1$ en utilisant la symétrie : $\left(y, p_{y}, \gamma\right) \mapsto\left(-y,-p_{y},-\gamma\right)$. On doit intégrer les équations suivantes :

$$
\begin{array}{lll}
\frac{d x}{d \tau}=\sqrt{c} P_{1} & \frac{d P_{1}}{d \tau}=y P_{2} P_{3} \\
\frac{d y}{d \tau}=P_{2} & \frac{d P_{2}}{d \tau}=-y P_{1} P_{3} \\
\frac{d z}{d \tau}=\frac{y^{2} \sqrt{c}}{2} P_{1} & \frac{d P_{3}}{d \tau}=0 .
\end{array}
$$

En introduisant $P_{3}=\lambda$ que l'on peut supposer $>0$ et l'angle $\theta: P_{1}=\cos \theta, P_{2}=\sin \theta$, l'équation dans le plan $(y, \theta)$ équivaut à

$$
\frac{d^{2} \theta}{d \tau^{2}}+\lambda \sin \theta=0
$$

et les géodésiques issues de 0 vérifient la condition $\frac{d \theta}{d \tau}(0)=y(0)=0$. Une telle solution de (3.3) est donc un pendule oscillant et via une reparamétrisation est donc équivalente au cas plat. Pour intégrer les équations on procède comme dans le cas plat, voir [1]. Introduisons $0<k, k^{\prime}<1$ en posant :

$$
k^{2}=\frac{1-\sin \varphi}{2}, k^{2}+k^{\prime 2}=1
$$


et écrivons l'équation caractéristique sous forme normale en utilisant l'homothétie: $\eta=\frac{y \sqrt{\lambda}}{2 k}$. On obtient donc :

$$
\left(\frac{d \eta}{\lambda d \tau}\right)^{2}=\left(1-\eta^{2}\right)\left(k^{\prime 2}+k^{2} \eta^{2}\right)
$$

que l'on intègre avec la condition initiale $\eta(0)=0$ et avec $\sigma=+1$, c'est à dire $\dot{\eta}(0)>0$. La variable $\eta$ oscille périodiquement entre -1 et +1 et se représente en utilisant les fonctions de Jacobi (voir [21]) sous la forme :

$$
\eta(\tau)=-\operatorname{cn}(K(k)+\tau \sqrt{\lambda}, k)
$$

où la période est $4 K(k)$ et $K(k)$ est l'intégrale elliptique complète de première espèce

$$
K(k)=\int_{0}^{1} \frac{d \eta}{\sqrt{\left(1-\eta^{2}\right)\left(k^{\prime 2}+k^{2} \eta^{2}\right)}} .
$$

On obtient donc :

$$
y(\tau)=-\frac{2 k}{\sqrt{\lambda}} \operatorname{cn}(K(k)+\tau \sqrt{\lambda}, k)
$$

où $t$ et $\tau$ sont reliés par :

$$
d t=(1+\gamma y) d \tau
$$

En utilisant la formule suivante ([21] p. 40) :

$$
\int \operatorname{cn} u d u=\frac{1}{k} \arcsin (k \operatorname{sn} u)
$$

on obtient :

$$
t=\tau-\frac{2 \gamma}{\lambda}[\arcsin (k \operatorname{sn}(K+\tau \sqrt{\lambda}))-\arcsin k] .
$$

En utilisant le formulaire de [21] concernant les fonctions de Jacobi, on peut ensuite calculer les coordonnées $x(),. z($.$) d'une géodésique normale e($.$) et l'on obtient le résultat suivant.$

Proposition 3.2. Les géodésiques normales issues de 0, paramétrisées par la longueur d'arc, associées à $\lambda>0$, $\dot{y}(0)>0$ et contenues dans un voisinage $U$ de 0 assez petit sont données par les formules suivantes :

$$
\begin{aligned}
x(t)= & -\tau+\frac{2}{\sqrt{\lambda}}(E(u)-E(K))-\frac{2 \gamma k}{\lambda}\left(\operatorname{sn} u \operatorname{dn} u-k^{\prime}\right) \\
y(t)= & -\frac{2 k}{\sqrt{\lambda}} \operatorname{cn} u \\
z(t)= & \frac{2}{3 \lambda^{3 / 2}}\left[\left(2 k^{2}-1\right)(E(u)-E(K))+k^{\prime 2} \tau \sqrt{\lambda}+2 k^{2} \operatorname{sn} u \operatorname{cn} u \operatorname{dn} u\right] \\
& -\frac{\gamma}{\lambda^{2}}\left[\arcsin (k \operatorname{sn} u)-\arcsin k+\left(2 k^{2}-1\right)\left(k \operatorname{sn} u \operatorname{dn} u-k k^{\prime}\right)+2 k^{3} \operatorname{cn}^{2} u \operatorname{sn} u \operatorname{dn} u\right] \\
t= & \tau-\frac{2 \gamma}{\lambda}[\arcsin (k \operatorname{sn} u)-\arcsin k]
\end{aligned}
$$

où $u=K+\tau \sqrt{\lambda}, 0<k, k^{\prime}<1, k^{2}=\frac{1-\sin \varphi}{2}, k^{2}+k^{\prime 2}=1$ et $\left.\varphi \in\right]-\frac{\pi}{2}, \frac{\pi}{2}[$. 
Remarque 3.3. Pour obtenir la paramétrisation des géodésiques associées à $\lambda>0, \dot{y}(0)<0$ on utilise la procédure suivante déduite du paragraphe 3.2, dans les formules précédentes on change $\gamma$ en $-\gamma$ pour obtenir $x, z, t$ et $y$ en $-y$. Par ailleurs les géodésiques qui correspondent à $\lambda<0$ se déduisent en utilisant la symétrie : $(x, y, z) \mapsto(-x, y,-z)$.

La variable $y$ oscille avec la période $4 K$ entre $y_{+}=\frac{2 k}{\sqrt{\lambda}}$ et $y_{-}=-\frac{2 k}{\sqrt{\lambda}}$. Aux deux premières intersections avec le plan $y=0$ associées à des temps $0<t_{1}<t_{2}$ on a les relations suivantes déduites de notre paramétrisation.

Corollaire 3.4. Les deux premières intersections d'une géodésique avec le plan $y=0$ vérifient :

$\star$ Première intersection : $\tau \sqrt{\lambda}=2 K$

$$
\begin{aligned}
& x\left(t_{1}\right)=-\tau+\frac{4}{\sqrt{\lambda}} E(K)+\frac{4 \gamma k k^{\prime}}{\lambda} \\
& z\left(t_{1}\right)=\frac{4}{3 \lambda^{3 / 2}}\left[\left(2 k^{2}-1\right) E(K)+k^{\prime 2} K\right]+\frac{2 \gamma}{\lambda^{2}}\left(\arcsin k+k k^{\prime}\left(2 k^{2}-1\right)\right) \\
& t_{1}=\tau+\frac{4 \gamma}{\lambda} \arcsin k . \\
& \star \\
& \text { Deuxième intersection }: \tau \sqrt{\lambda}=4 K \\
& x\left(t_{2}\right)=-t+\frac{8}{\sqrt{\lambda}} E(K) \\
& z\left(t_{2}\right)=\frac{8}{3 \lambda^{3 / 2}}\left[\left(2 k^{2}-1\right) E(K)+k^{\prime 2} K\right] \\
& t_{2}=\tau .
\end{aligned}
$$

\subsection{La nature de la sphère SR dans le cas plat}

Le cas plat joue un rôle important pour l'étude de la géométrie SR dans le cas Martinet, c'est en effet le modèle nilpotent d'ordre -1 . Il est donc important de rappeler ses propriétés essentielles décrites dans [1].

\subsubsection{Lieu conjugué et de coupure}

Dans la situation où la métrique est plate, $g=d x^{2}+d y^{2}$, le statut d'optimalité des géodésiques est très facile à décrire. Il y a en effet deux catégories de trajectoires.

- Géodésiques correspondant à $\lambda=0$. Ces géodésiques se projettent en des droites dans le plan $(x, y)$ et sont globalement minimisantes. Elles sont sans point conjugué excepté la géodésique anormale $a: t \mapsto( \pm t, 0,0)$ entièrement formée de points conjugués.

- Géodésiques correspondant à $\lambda \neq 0$. Pour ces géodésiques la coordonnée $y$ oscille avec une période $4 K$ dans la paramétrisation $t \sqrt{\lambda}$ entre deux valeurs symétriques $y_{-}<0<y_{+}$. Chaque géodésique admet un premier temps conjugué $t_{1 c}$ qui vérifie l'inégalité $2 K<t_{1 c} \sqrt{\lambda}<3 K$ et les simulations numériques montrent que $t_{1 c} \sqrt{\lambda} \sim 3 K$. Le point de coupure précède le point conjugué et est obtenu pour le temps $t_{c}$ vérifiant $t_{c} \sqrt{\lambda}=2 K$. Il correspond à la situation géométrique où la géodésique intersecte pour la première fois le plan de Martinet et le fait au même point que la géodésique déduite par la symétrie : $(x, y, z) \mapsto(x,-y, z)$.

Il résulte de ces propriétés que la trace de la sphère $S(0, r)$ avec le plan de Martinet est formée du lieu de coupure des géodésiques oscillantes de longueur $r$ et des extrémités : $x= \pm r, z=0$ de la géodésique anormale $a: t \mapsto( \pm t, 0,0)$. La représentation de cette trace montre que c'est une courbe simple dans le plan $y=0$ entourant le point 0 et analytique en dehors des points $x= \pm r, z=0$. Aux points $( \pm r, 0)$ il faut faire une étude des singularités. Par symétrie on peut supposer $z \geq 0$ et pour faire cette analyse on peut soit utiliser les formules intégrales générales du paragraphe 2 soit la représentation suivante, utilisant les fonctions de Jacobi, déduite du corollaire 3.4 :

$$
\begin{aligned}
& x=-r+2 r \frac{E}{K} \\
& z=\frac{r^{3}}{6 K^{3}}\left[\left(2 k^{2}-1\right) E+k^{\prime 2} K\right]
\end{aligned}
$$


où $0<k<1$ et $k$ désigne le module des fonctions elliptiques.

\subsubsection{Singularités du lieu de coupure}

Dans la représentation paramétrique précédente les singularités correspondent aux deux situations limites $k \rightarrow 0$ et $k \rightarrow 1$. Ces deux singularités sont très différentes.

En effet $K$ est solution de l'équation hypergéométrique (voir [21]) :

$$
k\left(1-k^{2}\right) \frac{d^{2} w}{d k^{2}}+\left(1-3 k^{2}\right) \frac{d w}{d k}-k w=0
$$

et représente lorsque $k \rightarrow 0$ une fonction analytique qui admet le développement suivant ([21] p. 73) :

$$
K=\frac{\pi}{2}\left[1+\left(\frac{1}{2}\right)^{2} k^{2}+\ldots\right], \quad k \rightarrow 0 .
$$

Par contre lorsque $k \rightarrow 1, k^{\prime}=\sqrt{1-k^{2}} \rightarrow 0$ et $K(k)$ admet un développement de la forme :

$$
K=u_{3}\left(k^{\prime}\right) \log \frac{4}{k^{\prime}}+u_{4}\left(k^{\prime}\right), \quad k^{\prime} \rightarrow 0
$$

où $u_{3}$ et $u_{4}$ sont des fonctions analytiques de la forme ([12] p. 134) :

$$
u_{3}\left(k^{\prime}\right)=1+\frac{k^{\prime 2}}{4}+o\left(k^{\prime 3}\right), \quad u_{4}\left(k^{\prime}\right)=-\frac{k^{\prime 2}}{4}+o\left(k^{\prime 3}\right) .
$$

De même $E$ est solution de l'équation hypergéométrique

$$
k\left(1-k^{2}\right) \frac{d w^{2}}{d k^{2}}+\left(1-k^{2}\right) \frac{d w}{d k}+k w=0
$$

et est lorsque $k \rightarrow 0$ une fonction analytique qui admet le développement suivant ([21] p. 73)

$$
E=\frac{\pi}{2}\left[1-\left(\frac{1}{2}\right)^{2} k^{2}-\ldots\right], \quad k \rightarrow 0 .
$$

Par contre lorsque $k \rightarrow 1, E(k)$ admet un développement donné par :

$$
E=u_{1}\left(k^{\prime}\right) \log \frac{4}{k^{\prime}}+u_{2}\left(k^{\prime}\right), \quad k^{\prime} \rightarrow 0
$$

où $u_{1}$ et $u_{2}$ sont des fonctions analytiques de la forme :

$$
u_{1}\left(k^{\prime}\right)=\frac{k^{\prime 2}}{2}+o\left(k^{\prime 3}\right), \quad u_{2}\left(k^{\prime}\right)=1-\frac{k^{\prime 2}}{4}+o\left(k^{\prime 3}\right) .
$$

L'explication géométrique est la suivante. L'intégrale elliptique complète $K$ représente le quart de la période du pendule oscillant et lorsque $k \rightarrow 0$, le comportement limite est celui d'un pendule linéarisé, et $K \rightarrow \frac{\pi}{2}$. Les fonctions de Jacobi de première espèce dégénèrent en des fonctions trigonométriques. Lorsque $k \rightarrow 1$, $K \rightarrow+\infty$ selon une échelle logarithmique et le comportement limite est celui d'un pendule au voisinage de la séparatrice joignant les cols. Les fonctions de Jacobi de première espèce se comportent alors comme des fonctions hyperboliques. 
En utilisant les développements lorsque $k \rightarrow 0$, on obtient que le lieu de coupure dans le secteur $z>0$ est un ensemble semi-analytique décrit comme une portion de graphe d'une fonction analytique dont le développement asymptotique est de la forme :

$$
z=-\frac{2 r^{2}}{3 \pi^{2}}(x-r)+o(x-r)
$$

L'analyse de la singularité lorsque $k \rightarrow 1$ requiert des outils plus sophistiqués car on sort de la catégorie analytique. On procède ainsi. Introduisons $X=\frac{x+r}{2 r}$ et le système (3.8) s'écrit :

$$
\begin{aligned}
& X=\frac{E}{K}=\frac{u_{1}\left(k^{\prime}\right) \log \frac{4}{k^{\prime}}+u_{2}\left(k^{\prime}\right)}{u_{3}\left(k^{\prime}\right) \log \frac{4}{k^{\prime}}+u_{4}\left(k^{\prime}\right)} \\
& z=\frac{r^{3}}{6 K^{3}}\left[\left(2 k^{2}-1\right) E+k^{\prime 2} K\right] .
\end{aligned}
$$

La propriété fondamentale est la suivante. L'équation (3.15) est une bijection locale au voisinage de $0^{+}$entre $X>0$ et $k^{\prime}>0$. En introduisant les variables :

$$
X_{1}=k^{\prime}, \quad X_{2}=\frac{1}{\log \frac{4}{k^{\prime}}}
$$

elle équivaut à l'équation :

$$
X=\frac{u_{1}\left(X_{1}\right)+X_{2} u_{2}\left(X_{1}\right)}{u_{3}\left(X_{1}\right)+X_{2} u_{4}\left(X_{1}\right)}
$$

Ce type d'équation appartient à la catégorie exp-log que l'on peut résoudre dans la même catégorie d'après [25]. Cela garantit sans calculs que le lieu de partage appartient à la catégorie exp-log et le même raisonnement est valide pour la sphère.

L'algorithme pratique de résolution est le suivant. La première étape est de trouver des équivalents pour $X_{1}$ et $X_{2}$ et le calcul montre que

$$
X_{1} \sim 4 \mathrm{e}^{-\frac{1}{x}}, \quad X_{2} \sim X
$$

On peut donc écrire :

$$
X_{1}=4 \mathrm{e}^{-\frac{1}{X}}\left(1+Y_{1}(X)\right), \quad X_{2}=X\left(1+Y_{2}(X)\right)
$$

où $Y_{1}, Y_{2} \rightarrow 0$ lorsque $X \rightarrow 0$. La seconde étape est de comparer les fonctions $Y_{1}$ et $Y_{2}$ et le calcul montre que :

$$
Y_{2}=X f\left(X, Y_{1}\right), \quad Y_{1} \sim \frac{Y_{2}}{X}, \quad X \rightarrow 0
$$

où $f$ est un germe de fonction analytique en 0 . En résolvant alors l'équation (3.17) en les variables $Y_{1}, X_{1}, X_{2}$ en utilisant le théorème des fonctions implicites dans la catégorie analytique le calcul montre que

$$
Y_{1}=h\left(X, \frac{\mathrm{e}^{-\frac{1}{X}}}{X^{2}}\right)
$$


où $h$ est un germe de fonction analytique en 0. En reportant ce résultat dans l'équation (3.16) on en déduit que lorsque $k \rightarrow 1$, le lieu de partage dans $z>0$ est paramétrisé par :

$$
\frac{z}{r^{3}}=F\left(X, \frac{\mathrm{e}^{-\frac{1}{X}}}{X^{2}}\right) .
$$

C'est ce type de calcul qu'il est fondamental de généraliser dans le cas intégrable. C'est une description qui précise exactement la transcendance du lieu de coupure.

On doit ensuite vérifier que ce lieu de coupure n'appartient pas à la famille sous-analytique du fait d'un phénomène de compensation. Cela se traduit par $F(X, Y)-F(X, 0)$ non identiquement nulle. Un calcul facile détaillé dans [1] montre le résultat suivant :

$$
\frac{z}{r^{3}}=\frac{1}{6} X^{3}-4 X^{3} \mathrm{e}^{-\frac{2}{X}}+o\left(X^{3} \mathrm{e}^{-\frac{2}{X}}\right)
$$

et cela prouve que le lieu de coupure n'est pas sous-analytique.

On résume ces propriétés dans la proposition suivante.

Proposition 3.5. Dans le cas plat la sphère n'est pas sous-analytique mais appartient à la catégorie exp-log. La trace de la sphère avec le plan de Martinet $y=0$ est une courbe simple fermée entourant 0 et symétrique par rapport à l'origine. Elle est formée des extrémités $x= \pm r, z=0$ de la géodésique anormale $a: t \rightarrow( \pm t, 0,0)$ de longueur $r$ et du lieu de coupure de longueur $r$. Dans le domaine $z \geq 0$ ce lieu de coupure est décrit par les graphes :

- au voisinage de $x=r, z=0$, c'est un graphe semi-analytique de la forme :

$$
z=-\frac{2 r^{2}}{3 \pi^{2}}(x-r)+o(x-r)
$$

- au voisinage de $x=-r, z=0$ c'est un graphe exp-log de la forme : $z=F\left(X, \frac{\mathrm{e}^{-\frac{1}{X}}}{X^{2}}\right)$ où $X=\frac{x+r}{2 r}$ et non sous-analytique car de la forme:

$$
z=\frac{r^{3}}{6} X^{3}-4 r^{3} X^{3} \mathrm{e}^{-\frac{2}{X}}+o\left(X^{3} \mathrm{e}^{-\frac{2}{X}}\right) .
$$

\subsection{Trace de la sphère et $\mathbf{d u}$ front d'onde de petit rayon avec le plan de Martinet dans le cas $\gamma$ quelconque}

\subsubsection{Préliminaires}

L'objectif de ce paragraphe est d'analyser la trace de la sphère $S(0, r)$ et du front d'onde $W(0, r)$ de petit rayon avec le plan de Martinet $y=0$. Pour cela on utilise deux techniques :

- évaluation de $S(0, r)$ et de $W(0, r)$ au voisinage de la géodésique anormale $a: t \rightarrow( \pm t, 0,0)$ en utilisant des développements asymptotiques (convergents)

- représentation de ces traces en utilisant la paramétrisation explicite des géodésiques à l'aide des fonctions de Jacobi et le package sur les fonctions elliptiques de Mathematica.

Notre étude se localise en 0 et on choisit le rayon $r$ assez petit de sorte que :

1. chaque géodésique de longueur $r$ soit paramétrisée par la proposition 3.2

2. chaque point à distance $\mathrm{SR} r$ de 0 peut-être atteint par une courbe minimisante.

On note $e:[0, T] \rightarrow \mathbf{R}^{3}$ une géodésique normale paramétrisée par la longueur d'arc, issue de 0 et associée à un paramètre $(\varphi, \lambda)$ du cylindre où $\varphi \neq \pm \frac{\pi}{2}$ et $\lambda>0$. Notons $0<t_{1}(\varphi, \lambda)<\ldots<t_{n}(\varphi, \lambda) \leq T$ les temps 
d'intersections successifs de $e($.$) avec le plan de Martinet y=0$. Si $e(t)=(x(t), y(t), z(t))$ et $\sigma=\operatorname{sign} \dot{y}(0)$, on pose

$$
\Gamma_{n}^{\sigma}(\gamma)=\cup_{\varphi \neq \frac{\pi}{2}, \lambda>0}\left(x\left(t_{n}(\varphi, \lambda)\right), z\left(t_{n}(\varphi, \lambda)\right)\right)
$$

et si la longueur est fixée à $r$ on utilise la notation $\Gamma_{n}^{\sigma}(\gamma, r)$. De notre analyse précédente, on déduit les propriétés suivantes.

\section{Lemme 3.6.}

- $\Gamma_{n}^{+}(0, r)=\Gamma_{n}^{-}(0, r)$ (cas plat)

- $\Gamma_{2 n+1}^{-}(\gamma, r)=\Gamma_{2 n+1}^{+}(-\gamma, r)$

- $\Gamma_{2 n}^{+}(\gamma, r)=\Gamma_{2 n}^{-}(\gamma, r)$.

On va décrire ces ensembles au voisinage du point $(-r, 0)$.

3.6.2. Description de $\Gamma_{1}^{+}(\gamma, r)(k \rightarrow 1)$

Théorème 3.7. L'ensemble $\Gamma_{1}^{+}(\gamma, r)$ est au voisinage de $X=0^{+}$avec $X=\frac{x+r}{2 r}$ un graphe de la forme :

$$
\frac{z}{r^{3}}=F\left(X, \frac{\mathrm{e}^{-\frac{1}{X}}}{X^{2}}, r \gamma\right)
$$

où $F$ est une fonction analytique d'un voisinage de $O_{\mathbf{R}^{3}}$ dans $\mathbf{R}$. De plus :

$$
F\left(X, \frac{\mathrm{e}^{-\frac{1}{X}}}{X^{2}}, r \gamma\right)=v_{1}(X, r \gamma)+v_{2}(X, r \gamma) \mathrm{e}^{-\frac{1}{X}}-4 \mathrm{e}^{-\frac{\pi r \gamma}{2}}(1+\pi r \gamma) X^{3} \mathrm{e}^{-\frac{2}{X}}+o\left(X^{3} \mathrm{e}^{-\frac{2}{X}}\right)
$$

où $v_{1}, v_{2}$ sont des fonctions analytiques et $v_{1}(X, r \gamma)=\frac{1}{6}\left(X^{3}-\frac{3}{4} r \gamma X^{3}+o\left(X^{4}\right)\right)$.

Corollaire 3.8. La trace de la sphère $S(0, r), r$ assez petit avec le plan de Martinet n'est pas sous-analytique.

Preuve. On peut supposer $\gamma>0$ et nos calculs montrent que la trace de la sphère avec $y=0$ dans le domaine $z \geq 0$ et au voisinage du point $(-r, 0)$ est formée de $\Gamma_{1}^{-}(\gamma, r) \cup( \pm r, 0)$. Pour $r$ assez petit, $1+\pi r \gamma$ est non nul et la sphère n'est pas semi-analytique.

Remarque 3.9. Le cas plat est obtenu par continuité en faisant $\gamma=0$ dans les formules.

Preuve. Preuve du théorème 3.7.

Considérons l'équation (voir Cor. 3.4)

$$
\frac{x+r}{2 r}=\frac{\tau E}{r K}+\left(\frac{\tau}{r K}\right)^{2} \frac{r \gamma}{2}\left(k k^{\prime}+\arcsin k\right)
$$

où

$$
\tau=r-\frac{4 \gamma}{\lambda} \arcsin k
$$

et

$$
\tau \sqrt{\lambda}=2 K
$$

Introduisons les coordonnées suivantes :

$$
X=\frac{x+r}{2 r}, X_{1}=k^{\prime}, \quad X_{2}=\frac{1}{\log \frac{4}{k^{\prime}}}
$$


et notons :

$$
f=4 r \gamma \arcsin k, \varepsilon=r \gamma .
$$

Comme dans le cas plat notre objectif est de résoudre (3.22) c'est-à-dire de calculer $k^{\prime}$ et donc $\frac{1}{\log \frac{4}{k^{\prime}}}$ en fonction de $X$, au voisinage de $0^{+}$. En utilisant (3.23) et (3.24) on obtient la relation :

$$
\tau=r-\gamma\left(\frac{\tau}{K}\right)^{2} \arcsin k
$$

En résolvant cette équation du second degré en $\frac{\tau}{r K}$ avec $k \rightarrow 1$ et $\tau \sim r$, il vient

$$
\frac{\tau}{r K}=\frac{-K+K \sqrt{1+\frac{4 r \gamma \arcsin k}{K^{2}}}}{2 r \gamma \arcsin k}
$$

et $\frac{\tau}{r K}$ admet donc un développement convergent de la forme :

$$
\frac{\tau}{r K}=\frac{1}{K}-\frac{f}{4 K^{3}}+O\left(\frac{f^{2}}{K^{5}}\right) .
$$

De plus puisque $k^{\prime}=\sqrt{1-k^{2}}$, arcsin $k$ est au voisinage de $k^{\prime}=0$ une fonction analytique de $k$ qui admet le développement suivant :

$$
\arcsin k=\frac{\pi}{2}-k^{\prime}-\frac{5}{24} k^{\prime 3}+O\left(k^{\prime 5}\right) .
$$

Donc $f=4 r \gamma \arcsin k$ est une fonction analytique en $\varepsilon=r \gamma$ et $k^{\prime}$ au voisinage de 0 .

D'autre part en utilisant les notations du paragraphe 3.5.2 :

$$
\frac{1}{K}=\frac{1}{u_{3}\left(k^{\prime}\right) \log \frac{4}{k^{\prime}}+u_{4}\left(k^{\prime}\right)}=\frac{1}{\log \frac{4}{k^{\prime}}}\left(\frac{1}{u_{3}\left(k^{\prime}\right)+\frac{u_{4}\left(k^{\prime}\right)}{\log \frac{4}{k^{\prime}}}}\right)
$$

et admet le développement convergent en 0 suivant :

$$
\frac{1}{K}=\frac{X_{2}}{1+\frac{k^{\prime 2}}{4}+o\left(k^{\prime 3}\right)+X_{2}\left(-\frac{k^{\prime 2}}{4}+o\left(k^{\prime 3}\right)\right)}=X_{2}\left(1+O\left(X_{1}^{2}\right)+X_{2} O\left(X_{1}^{2}\right)\right) .
$$

De même :

$$
\frac{E}{K}=\frac{u_{1}\left(k^{\prime}\right) \log \frac{4}{k^{\prime}}+u_{2}\left(k^{\prime}\right)}{u_{3}\left(k^{\prime}\right) \log \frac{4}{k^{\prime}}+u_{4}\left(k^{\prime}\right)}
$$

et admet le développement convergent en 0 suivant :

$$
\frac{E}{K}=X_{2}+O\left(X_{1}^{2}\right)+X_{2} O\left(X_{1}^{2}\right) .
$$

L'équation (3.22) s'écrit donc sous la forme:

$$
X=A_{1}\left(X_{1}, X_{2}, r \gamma\right)
$$


où $A_{1}$ est une fonction analytique en 0 et admet le développement convergent suivant :

$$
X=X_{2}+\frac{r \gamma \pi}{4} X_{2}^{2}+O\left(X_{1}^{2}\right)+R
$$

où $R$ est une fonction analytique dont le développement est d'ordre 3 en les variables $X_{1}, X_{2}$.

En inversant l'équation précédente il vient :

$$
X_{2}=X-\frac{r \gamma \pi}{4} X^{2}+O\left(X_{1}^{2}\right)+R^{\prime}
$$

où $R^{\prime}$ est une fonction analytique dont le développement est d'ordre 3 en les variables $X, X_{1}$.

D'autre part, par définition

$$
X_{1}=4 \mathrm{e}^{-\frac{1}{X_{2}}}
$$

donc $X_{1}=o\left(X_{2}\right)$ lorsque $X_{2} \rightarrow 0$. On en déduit que $X_{2} \sim X$ lorsque $X \rightarrow 0$ et on peut écrire

$$
X_{2}=X\left(1+Y_{2}\right)
$$

avec $Y_{2} \rightarrow 0$ lorsque $X \rightarrow 0$ et

$$
Y_{2}=-\frac{r \gamma \pi}{4} X+\frac{O\left(X_{1}^{2}\right)}{X}+\frac{R^{\prime}}{X}
$$

Posons

$$
\hat{Y}_{2}=Y_{2}+\frac{r \gamma \pi}{4} X
$$

on a donc :

$$
\frac{\hat{Y}_{2}}{X}=O(X) \text { lorsque } X \rightarrow 0
$$

Par ailleurs :

$$
X_{1}=4 \mathrm{e}^{-\frac{1}{X_{2}}}=4 \mathrm{e}^{-\frac{1}{X\left(1+Y_{2}\right)}}=4 \mathrm{e}^{-\frac{1}{X\left(1-\frac{r \gamma \pi}{4} X+Y_{2}\right)}}=4 \mathrm{e}^{-\frac{r \gamma \pi}{4}} \mathrm{e}^{-\frac{1}{X}}\left(1+Y_{1}\right)
$$

et donc

$$
Y_{1}=A_{2}\left(\frac{\hat{Y}_{2}}{X}, X, r \gamma\right)
$$

où $A_{2}$ est une fonction analytique en 0 et d'autre part :

$$
Y_{1}=O(X) \text { lorsque } X \rightarrow 0
$$

et

$$
\frac{\hat{Y}_{2}}{X}=A_{3}\left(Y_{1}, X, r \gamma\right)
$$

où $A_{3}$ est une fonction analytique en 0 et $\frac{\partial A_{3}}{\partial Y_{1}}(0)=1$. 
L'équation à résoudre s'écrit donc après simplification sous la forme

$$
X=X\left(1+Y_{2}+\frac{A_{5}\left(X_{1}, X_{2}, r \gamma\right)}{X}\right)=X\left(1+\hat{Y}_{2}+\frac{A_{6}\left(X_{1}, X_{2}, r \gamma\right)}{X}\right)
$$

où $A_{5}, A_{6}$ sont analytiques en 0 et $A_{6}$ est d'ordre 3 en $X_{2}$ d'après (3.26). Après simplification par $X^{2}$ on doit donc résoudre une équation de la forme

$$
\frac{\hat{Y}_{2}}{X}+\frac{A_{6}\left(X_{1}, X_{2}, r \gamma\right)}{X^{2}}=0
$$

qui s'écrit

$$
A_{7}\left(Y_{1}, X, \frac{\mathrm{e}^{-\frac{1}{x}}}{X^{2}}, r \gamma\right)=0
$$

où $A_{7}$ est analytique en $0, A_{7}(0)=0$ et $\frac{\partial A_{7}}{\partial Y_{1}}(0)=1$. En utilisant le théorème des fonctions implicites dans la catégorie analytique on en déduit que

$$
Y_{1}=A_{8}\left(X, \frac{\mathrm{e}^{-\frac{1}{x}}}{X^{2}}, r \gamma\right)
$$

où $A_{8}$ est analytique en 0.

En conclusion $X_{1}=k^{\prime}$ et $X_{2}=\frac{1}{\log \frac{4}{k^{\prime}}}$ sont des fonctions analytiques en 0 par rapport aux variables $X, \frac{\mathrm{e}^{-\frac{1}{X}}}{X^{2}}, r \gamma$.

Par ailleurs avec $Z=\frac{z}{r^{3}}$ on a :

$$
Z=\frac{1}{6}\left(\frac{\tau}{r K}\right)^{3}\left[\left(2 k^{2}-1\right) E+k^{\prime 2} K\right]+\frac{1}{8}\left(\frac{\tau}{r K}\right)^{4}(r \gamma)\left(\arcsin k+k k^{\prime}\left(2 k^{2}-1\right)\right)
$$

et on montre comme précédemment que $Z=A_{9}\left(X_{1}, X_{2}, r \gamma\right)$ où $A_{9}$ est une fonction analytique en 0 et donc

$$
Z=F\left(X, \frac{\mathrm{e}^{-\frac{1}{X}}}{X^{2}}, r \gamma\right)
$$

où $F$ est analytique en 0 .

Algorithme : En posant

$$
F\left(X, \frac{\mathrm{e}^{-\frac{1}{X}}}{X^{2}}, r \gamma\right)=\sum_{i=0}^{+\infty} u_{i}(X, r \gamma)\left(\frac{\mathrm{e}^{-\frac{1}{X}}}{X^{2}}\right)
$$




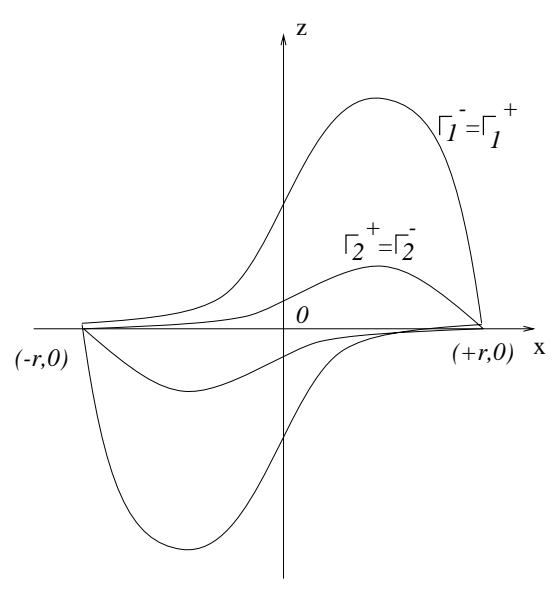

FiguRE 5. $\gamma=0$.

notre procédure permet de calculer formellement les coefficients des fonctions $u_{i}$. En effet posons :

$$
\begin{aligned}
Z & =\sum_{n, p \geq 0} B_{n, p} X_{1}^{n} X_{2}^{p} \\
X_{1} & =\mathrm{e}^{-\frac{1}{X}}\left(\alpha+\sum_{n \geq 0} c_{n}(X)\left(\frac{\mathrm{e}^{-\frac{1}{X}}}{X^{2}}\right)^{n}\right) \\
X_{2} & =X\left(1+\sum_{n \geq 0} d_{n}(X)\left(\frac{\mathrm{e}^{-\frac{1}{X}}}{X^{2}}\right)^{n}\right) .
\end{aligned}
$$

La partie analytique de $Z$ est donnée par :

$$
u_{1}(X)=\sum_{p \geq 0} B_{0, p} X^{p}\left(1+d_{0}(X)\right)^{p}
$$

et on calcule de même tous les $u_{i}$.

En particulier en appliquant cet algorithme on obtient le développement de $F$ décrit dans le théorème.

3.6.3. Trace de la sphère et du front d'onde de petit rayon avec $y=0$

À l'aide de notre paramétrisation et de nos calculs asymptotiques on peut représenter l'intersection de la sphère et du front d'onde de petit rayon avec le plan $y=0$. On peut par symétrie supposer $\gamma \geq 0$.

Pour $\gamma>0$, les deux courbes $\Gamma_{1}^{\sigma}(\gamma, r)$ sont distinctes et la trace $S(0, r) \cap\{y=0\}$ est l'union de $\Gamma_{1}^{-}(\gamma, r)$ et de sa symétrique $-\Gamma_{1}^{-}(\gamma, r)$ avec les deux points $(x, z)=( \pm r, 0)$ extrémités de la géodésique anormale de longueur $r$. Cet ensemble est une courbe fermée entourant le domaine $\cup_{n \geq 1} \Gamma_{n}^{\sigma}(\gamma, r) \cap\{y=0\}$. Quand $\gamma=0$, les deux courbes $\Gamma_{1}^{ \pm}(0, r)$ sont les mêmes.

On représente sur les figures $5,6,7$ la trace du front d'onde dans le cas plat et le cas non plat, en limitant notre représentation aux deux premières intersections $\Gamma_{1}^{\sigma}, \Gamma_{2}^{\sigma}$. 


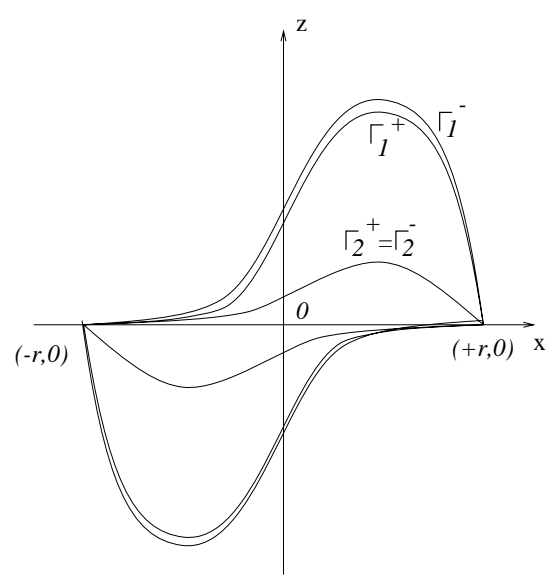

FiguRE $6 . \gamma \neq 0$.

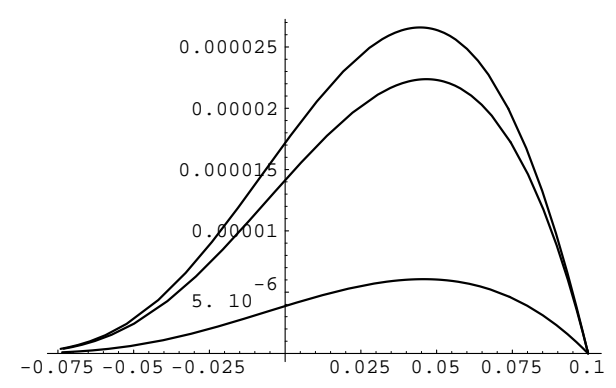

FiguRE 7. Simulations numériques, $z>0: \gamma>0$.

Dans le cas plat, chaque point $P$ distinct de $( \pm r, 0)$ de $W(0, r) \cap\{y=0\}$ est l'extrémité de deux géodésiques distinctes associées à $\sigma=+1$ et $\sigma=-1$. En particulier le lieu de coupure est précisement $\pm \Gamma_{1}^{+}(0, r)$. Quand $\gamma \neq 0$, cette propriété n'est plus vraie et le plan $y=0$ ne contient aucun point de coupure. Néanmoins chaque point de $\pm \Gamma_{2}^{+}(\gamma, r)$ est l'extrémité de deux géodésiques de même longueur.

Comme dans le cas plat, le front d'onde se ramifie aux 2 points $( \pm r, 0)$. En chaque point il y a une infinité de branches. En particulier sa trace avec la droite $x=0$ admet une infinité de composantes connexes. Il n'appartient donc à aucune catégorie raisonnable de la géométrie analytique. Chaque branche dans le domaine $z \geq 0$ est semi-analytique en $(r, 0)$ mais non sous-analytique en $(-r, 0)$ tout en appartenant à la catégorie explog. Un calcul facile montre que les termes plats jouent peu de rôle, le contact entre les branches se caractérisant à partir de leur partie analytique. Ce sont d'ailleurs des invariants donnés par le cas plat.

\subsection{Conclusion : Le rôle du paramètre $\gamma$}

Notre étude montre que le paramètre $\gamma$ bien que brisant la symétrie $(x, y, z) \mapsto(x,-y, z)$ du cas plat a peu d'influence sur la nature de la sphère. En effet, il peut être absorbé par un changement de paramétrage et seules les trajectoires oscillantes du pendule jouent un rôle pour le calcul de la sphère et du front d'onde. Le feuilletage $(\mathcal{F})$ associé au problème et la contrainte $(\mathcal{C})$ étant identiques à ceux du cas plat on a :

$$
\theta^{\prime \prime}+\lambda \sin \theta=0, \quad \theta^{\prime}=0 .
$$

La projection de l'application retour $R$ dans le plan de phase 
$\left(\theta, \theta^{\prime}\right)$ est

- $n$ impair : $R_{n}^{\theta}:(\theta, 0) \mapsto(-\theta, 0)$

- $n$ pair : $R_{n}^{\theta}=i d$.

La trace de la sphère avec le plan $y=0$ nous donne des renseignements précis sur l'optimalité des géodésiques. En particulier la sphère se construit à partir de l'image de l'application premier retour et on obtient un encadrement des points de coupure. Le calcul exact du lieu conjugué et du lieu de coupure peut être réalisé. Pour le lieu conjugué le calcul est identique à celui du cas plat décrit dans [1]. Pour le lieu de coupure le calcul est plus complexe et comme pour la trace de la sphère avec $y=0$ il faut résoudre des équations dans la catégorie exp-log.

\section{Analyse du RÔle paramètre $\alpha$}

\subsection{Préliminaires géométriques}

Notre étude du paragraphe 2.6.2 montre que l'on peut ramener l'étude de la déformation à un paramètre : $g=(1+\alpha y)^{2} d x^{2}+d y^{2}$ à étudier l'équation d'un pendule $\theta^{\prime \prime}+\sin \theta=0$ en utilisant essentiellement une homographie sur $y$. Cette homographie rend les calculs plus complexes. Pour simplifier cela on va utiliser une déformation à un paramètre du cas plat où le feuilletage $(\mathcal{F})$ est déjà un pendule.

\subsection{Modèle}

Considérons la déformation à un paramètre suivante de la forme de Martinet :

$$
\omega=(1+\varepsilon y) d z-\frac{y^{2}}{2} d x
$$

La distribution $D$ est engendrée par :

$$
F_{1}=(1+\varepsilon y) \frac{\partial}{\partial x}+\frac{y^{2}}{2} \frac{\partial}{\partial z}, \quad F_{2}=\frac{\partial}{\partial y} .
$$

En calculant il vient :

$$
\left[F_{1}, F_{2}\right]=\varepsilon \frac{\partial}{\partial x}+y \frac{\partial}{\partial z}, \quad\left[\left[F_{1}, F_{2}\right], F_{2}\right]=\frac{\partial}{\partial z}, \quad\left[\left[F_{1}, F_{2}\right], F_{1}\right]=0
$$

et les crochets de longueur $\geq 4$ sont nuls. L'algèbre de Lie engendrée par $F_{1}, F_{2}$ est donc nilpoptente. Le lieu singulier $S: \operatorname{det}\left(F_{1}, F_{2},\left[F_{1}, F_{2}\right]\right)=0$ où $\omega$ n'est pas une forme de contact est donné par : $y\left(1+\varepsilon \frac{y}{2}\right)=0$. Donc $S$ est l'union des deux plans $y=0$ et $y=-\frac{2}{\varepsilon}$. On localise notre étude dans un voisinage de 0 et l'on va donc supposer :

$\left(H_{1}\right) \quad|y \varepsilon|<2$

et dans ce domaine la surface de Martinet est le plan $y=0$.

On définit la métrique $g$ sur $D$ en convenant que les deux champs $F_{1}$ et $F_{2}$ sont orthonormés. $\mathrm{Si}(1+\varepsilon y) \neq 0$, on peut écrire sur $D: d z=\frac{y^{2}}{2(1+\varepsilon y)} d x$. On va donc se restreindre au domaine $U$ donné par :

$\left(H_{2}\right) \quad|y \varepsilon|<1$

où la métrique $\mathrm{SR}$ est représentée par :

$$
g=\frac{d x^{2}}{(1+\varepsilon y)^{2}}+d y^{2}
$$

et $g_{R}$ désigne la métrique riemannienne induite sur le plan $(x, y)$.

Il est intéressant de noter que ce type de déformation du cas plat a été choisi dans [26] pour montrer l'optimalité des géodésiques strictement anormales en géométrie SR. 


\subsection{Equations des géodésiques dans $U$}

\subsubsection{Géodésiques anormales}

Le système s'écrit

$$
\begin{aligned}
& \dot{x}=u_{1}(1+\varepsilon y) \\
& \dot{y}=u_{2} \\
& \dot{z}=u_{1} \frac{y^{2}}{2} .
\end{aligned}
$$

Le contrôle associé aux géodésiques anormales vérifie $u_{2}(t) \equiv 0$ et les géodésiques anormales sont contenues dans le plan de Martinet. Elles vérifient les équations :

$$
\dot{x}=u_{1}, \quad y=0, \quad z=z_{0}
$$

et ce sont des droites. On note $a: t \mapsto( \pm t, 0,0)$ la géodésique anormale issue de 0 et paramétrisée par la longueur d'arc. D'après [1] et [26] c'est une géodésique globalement minimisante si sa longueur est assez petite. Son extrémité $x= \pm r, y=z=0$ appartient donc à $S(0, r)$ si $r$ est assez petit.

\subsubsection{Géodésiques normales}

On pose $F_{3}=\frac{\partial}{\partial z}$ et $P_{i}=\left\langle p, F_{i}(q)\right\rangle$ pour $i=1,2,3$. Le hamiltonien associé aux relèvements géodésiques normaux est $H_{n}=\frac{1}{2}\left(P_{1}^{2}+P_{2}^{2}\right)$. On observe que $x$ et $z$ sont des coordonées cycliques et $p_{x}, p_{z}$ sont les intégrales premières associées. Les relèvements géodésiques normaux vérifient

$$
\begin{aligned}
\dot{x} & =(1+\varepsilon y)\left[p_{x}(1+\varepsilon y)+p_{z} \frac{y^{2}}{2}\right] \\
\dot{y} & =p_{y} \\
\dot{z} & =\frac{y^{2}}{2}\left[p_{x}(1+\varepsilon y)+p_{z} \frac{y^{2}}{2}\right] \\
\dot{p}_{x} & =0 \\
\dot{p}_{y} & =-\left(\varepsilon p_{x}+p_{z} y\right)\left[p_{x}(1+\varepsilon y)+p_{z} \frac{y^{2}}{2}\right] \\
\dot{p}_{z} & =0 .
\end{aligned}
$$

Dans les coordonnées $(q, P)$ il vient :

$$
\begin{aligned}
\dot{x} & =(1+\varepsilon y) P_{1} \\
\dot{y} & =P_{2} \\
\dot{z} & =\frac{y^{2}}{2} P_{1} \\
\dot{P}_{1} & =\left[y P_{3}+\frac{\varepsilon}{1+\varepsilon y}\left(P_{1}-\frac{y^{2}}{2} P_{3}\right)\right] P_{2} \\
\dot{P}_{2} & =-\left[y P_{3}+\frac{\varepsilon}{1+\varepsilon y}\left(P_{1}-\frac{y^{2}}{2} P_{3}\right)\right] P_{1} \\
\dot{P}_{3} & =0 .
\end{aligned}
$$




\subsection{Géodésiques de la métrique induite}

On les calcule aisément en posant $p_{z}=0$ dans les équations précédentes. Alors $P_{1}=p_{x}(1+\varepsilon y), P_{2}=p_{y}$ et les géodésiques de la métrique induite $g_{R}$ vérifient :

$$
\begin{aligned}
\dot{x} & =(1+\varepsilon y) P_{1} \\
\dot{y} & =P_{2} \\
\dot{P}_{1} & =\frac{\varepsilon}{1+\varepsilon y} P_{1} P_{2} \\
\dot{P}_{2} & =-\frac{\varepsilon}{1+\varepsilon y} P_{1}^{2} .
\end{aligned}
$$

Elles peuvent s'étudier à l'aide de l'équation caractéristique :

$$
\dot{y}^{2}+P_{1}^{2}(y)=1
$$

où le potentiel $P_{1}^{2}$ est quadratique. L'espace physique est $\left|P_{1}(y)\right| \leq 1$ et on intègre avec la condition initiale $y(0)=0$. En particulier $\left|p_{x}\right| \leq 1$. L'intégration est élémentaire en écrivant l'équation caractéristique sous la forme de l'équation linéaire :

$$
\ddot{y}+\varepsilon p_{x}^{2}(1+\varepsilon y)=0 .
$$

Pour $\varepsilon \neq 0$, les solutions issues de 0 sont toutes périodiques excepté le cas $p_{x}=0$ où l'on obtient le résultat suivant.

Lemme 4.1. La droite géodésique paramétrisée par la longueur d'arc $\bar{e}: x=z=0, y= \pm t$ est la seule géodésique paramétrisée par la longueur d'arc e $:[0, T] \rightarrow U, e=(x, y, z)$ telle que $e(0)=0$ et $e(T)=(0, *, 0)$.

Preuve. La preuve est immédiate en utilisant la relation :

$$
\frac{d}{d t}\left(p_{x} x+p_{z} z\right)=P_{1}^{2} .
$$

Remarque 4.2. Les extrémités des géodésiques de longueur $r$ associées à $\lambda=0$ font partie de la sphère $S(0, r)$ si $r$ est assez petit. Cela définit donc une courbe paramétrisée à l'aide des fonctions élémentaires contenue sur la sphère, dont les points $y= \pm r, x=z=0$ extrémités de $\bar{e}($.$) .$

\subsection{Classification des géodésiques normales contenues dans $U$ et associées à $p_{z} \neq 0$}

\subsubsection{Notations et symétries}

On observe que les équations (4.1) et la métrique $g$ sont laissées invariantes par la transformation : $X=$ $-x, Y=y, Z=-z, P_{X}=-p_{x}, P_{Y}=p_{y}, P_{Z}=-p_{z}$ et donc l'ensemble des géodésiques normales, la sphère $S(0, r)$ et le front d'onde $W(0, r)$ sont laissés invariants par la symétrie $S:(x, y, z) \mapsto(-x, y,-z)$. On note $e:[0, T] \rightarrow U$ une géodésique normale issue de $0, e(t)=(x(t), y(t), z(t))$ paramétrisée par la longueur d'arc. Elle dépend des paramètres suivants : $P_{1}(0)=p_{x}=\sin \varphi, P_{2}(0)=p_{y}(0)=\cos \varphi$ où $\varphi \in\left[0,2 \pi\left[\right.\right.$ et $P_{3}=\lambda \neq 0$. En utilisant la symétrie précédente on peut restreindre notre étude au cas $\lambda>0$. De plus en adjoignant à l'équation (4.1) l'équation $\dot{\varepsilon}=0$ pour former un système (4.1)', ce système et la métrique sont laissés invariants par la transformation $\left(x, y, z, p_{x}, p_{y}, p_{z}, \varepsilon\right) \mapsto\left(x,-y, z, p_{x},-p_{y}, p_{z},-\varepsilon\right)$. On peut donc fixer dans notre étude le signe de $\varepsilon$ et on choisit : $\varepsilon \leq 0$. 


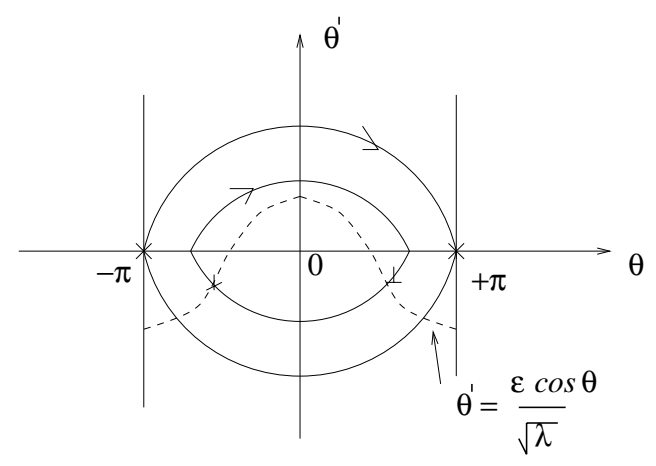

Figure 8

\subsubsection{Le pendule}

En posant $P_{1}(t)=\cos \theta(t), P_{2}(t)=\sin \theta(t)$ dans les équations (4.2) il vient avec $P_{1}=p_{x}(1+\varepsilon y)+p_{z} \frac{y^{2}}{2}$ :

$$
-\sin \theta \dot{\theta}=\left(p_{x} \varepsilon+p_{z} y\right) \dot{y}
$$

et avec $\dot{y}=P_{2}$ on obtient si $\theta \neq k \pi$ l'équation :

$$
\dot{\theta}=-\left(p_{x} \varepsilon+p_{z} y\right)
$$

et en dérivant il vient l'équation du pendule :

$$
\ddot{\theta}+\lambda \sin \theta=0 .
$$

En introduisant $s=t \sqrt{\lambda}$ et en notant ' la dérivée par rapport à $s$ on peut écrire (4.6) comme :

$$
\theta^{\prime \prime}+\sin \theta=0
$$

C'est l'équation du pendule dont les solutions $\theta^{\prime}$ se paramétrisent (voir [21] p. 115) à l'aide des fonctions de Jacobi : cn et dn. En intégrant avec la condition initiale $y(0)=0$ il vient $\dot{\theta}(0)=-p_{x} \varepsilon$ soit :

$$
\theta^{\prime}(0)=-\frac{p_{x} \varepsilon}{\sqrt{\lambda}}=-\frac{\varepsilon \cos \theta(0)}{\sqrt{\lambda}}
$$

Dans le cas plat on n'obtient que les solutions oscillantes, car $\theta^{\prime}(0)=0$, paramétrisées par la fonction cn mais si $\varepsilon \neq 0$ on obtient également des solutions en rotation.

On peut observer que la géodésique anormale associée à $\theta(t)=k \pi$ se projette en les positions d'équilibre du pendule, néanmoins ce n'est pas la projection d'une géodésique normale.

Cette étude permet de ramener géométriquement l'étude des géodésiques à l'intégration de :

$$
\theta^{\prime \prime}+\sin \theta=0, \quad \theta^{\prime}(0)=-\frac{\varepsilon \cos \theta(0)}{\sqrt{\lambda}} .
$$

Dans le cas plat, l'ensemble $y=0$ dégénère en la droite $\theta^{\prime}=0$.

On peut obtenir la paramétrisation de $y$ défini par (4.5) à l'aide des fonctions de Jacobi en utilisant les formules de [21]. On peut aussi le faire en mettant l'équation caractéristique sous forme normale et c'est l'objet du paragraphe suivant. 


\subsubsection{Equation caractéristique sous forme normale}

L'équation caractéristique s'écrit :

$$
\dot{y}^{2}+P_{1}^{2}(y)=1
$$

où $P_{1}(y)=p_{x}(1+\varepsilon y)+p_{z} \frac{y^{2}}{2}$ et $p_{x}, p_{z}$ sont des constantes. L'espace physique est $\left\{y \in U,\left|P_{1}(y)\right| \leq 1\right\}$ et avec $y(0)=0$ il vient $\left|p_{x}\right| \leq 1$. On pose $p_{x}=\sin \varphi, p_{z}=\lambda>0$ et l'on peut écrire :

$$
1-P_{1}=1-p_{x}+\frac{\varepsilon^{2} p_{x}^{2}}{2 \lambda}-\frac{\lambda}{2}\left(y+\frac{\varepsilon p_{x}}{\lambda}\right)^{2} .
$$

Puisque $\left|p_{x}\right| \leq 1$, on a : $1-p_{x}+\frac{\varepsilon^{2} p_{x}^{2}}{2 \lambda}>0$ quand $\varepsilon \neq 0$. Si $\varepsilon=0$, on est dans le cas plat et l'on peut supposer $\varphi \neq \pm \frac{\pi}{2} \operatorname{car} \varphi=\frac{\pi}{2}$ correspond à la direction anormale, i.e. $\left|p_{x}\right|=1$. Donc si l'on pose :

$$
2 k^{2}=1-p_{x}+\frac{\varepsilon^{2} p_{x}^{2}}{2 \lambda}
$$

où $k \geq 0$, on peut toujours supposer $2 k^{2}>0$. De même

$$
1+P_{1}=1+p_{x}-\frac{\varepsilon^{2} p_{x}^{2}}{2 \lambda}+\frac{\lambda}{2}\left(y+\frac{\varepsilon p_{x}}{\lambda}\right)^{2}
$$

et l'on introduit :

$$
2 k^{\prime \prime}=1+p_{x}-\frac{\varepsilon^{2} p_{x}^{2}}{2 \lambda}
$$

et l'on a : $k^{2}+k^{\prime \prime}=1$. Soit $k^{\prime} \geq 0$ défini par $k^{\prime 2}=\left|k^{\prime \prime}\right|$, on a donc $: k^{\prime \prime}=\delta k^{\prime 2}$ où $\delta$ est une constante égale à $0,1 \mathrm{ou}-1$. L'équation caractéristique prend la forme normale

$$
\dot{y}^{2}=4 k^{2}\left(1-\eta^{2}\right)\left(k^{\prime \prime}+k^{2} \eta^{2}\right)
$$

où l'on a posé

$$
\eta=\frac{1}{2 k}\left(y \sqrt{\lambda}+\frac{\varepsilon p_{x}}{\sqrt{\lambda}}\right) .
$$

On observe que l'on peut mettre l'équation caractéristique sous forme normale en utilisant la similitude définie par (4.11), voir la discussion du paragraphe 4.1 pour traiter le cas général et le sens de notre modèle.

Dans la coordonnée normalisée $\eta$ l'équation s'écrit donc

$$
\frac{\dot{\eta}^{2}}{\lambda}=\left(1-\eta^{2}\right)\left(k^{\prime \prime}+k^{2} \eta^{2}\right) .
$$

\subsubsection{Forme du potentiel $P_{1}^{2}$}

Le comportement des solutions de l'équation caractéristique peut être analysé en considérant le graphe de $P_{1}(y)$ en dehors de toute normalisation. Observons que

$$
P_{1}=p_{x}(1+\varepsilon y)+\lambda \frac{y^{2}}{2}
$$


où $P_{1}(0)=p_{x},\left|p_{x}\right| \leq 1$. Quand $y \rightarrow \infty, P_{1} \rightarrow+\infty$ et $P_{1}$ possède un unique minimum : $m=-\frac{\varepsilon p_{x}}{\lambda}$ et $P_{1}(m)=p_{x}-\frac{\varepsilon^{2} p_{x}^{2}}{2 \lambda}$.

Notons $y_{1}<y_{2}$ les solutions de $P_{1}(y)=1$, c'est-à-dire les racines de

$$
\lambda \frac{y^{2}}{2}+\varepsilon p_{x} y+\left(p_{x}-1\right)=0 .
$$

Le discriminant est $\Delta=4 \lambda k^{2}>0$ et $y_{1} y_{2}=\frac{2\left(p_{x}-1\right)}{\lambda} \leq 0$. Si $p_{x}=1$ et $\varepsilon<0$ on est dans le cas limite : $y_{1}=0, y_{2}=-\frac{2 \varepsilon}{\lambda}>0$. De plus $P_{2}(0)=0, \dot{y}(0)=\hat{P}_{2}(0)=0, \ddot{y}(0)=\dot{P}_{2}(0)=-\varepsilon P_{1}^{2}(0)>0$. De notre étude on déduit le résultat suivant.

Lemme 4.3. Supposons $\varepsilon<0$. Les racines de $P_{1}(y)=1$ sont deux points distincts $y_{1}<y_{2}$ et $0 \in\left[y_{1}, y_{2}\right]$. Le mouvement $y(t)$ où $y(0)=0$ est confiné au segment $\left[y_{1}, y_{2}\right]$. Si $p_{x} \neq 1$, on a $\left.0 \in\right] y_{1}, y_{2}\left[\right.$ et si $p_{x}=1$ alors $y_{1}=0$.

Dans la coordonnée normalisée, les racines $y_{1}, y_{2}$ correspondent à $\eta= \pm 1$. Calculons maintenant les racines de $P_{1}(y)=-1$ qui correspondent alors à $\eta= \pm \sqrt{\frac{-k^{\prime \prime}}{k^{2}}}$. Ce sont les solutions de l'équation :

$$
\lambda \frac{y^{2}}{2}+\varepsilon p_{x} y+\left(p_{x}+1\right)=0
$$

dont le discriminant est $\Delta^{\prime \prime}=-4 \lambda k^{\prime \prime}$. Il y a une situation critique pour $k^{\prime \prime}=0$ où $P_{1}(m)=-1$ et la racine est double. On distingue donc trois cas :

1. Cas $\mathrm{A}: k^{\prime \prime}>0$ et $P_{1}(y)=-1$ n'a pas de racine réelle.

2. Cas $\mathrm{B}: k^{\prime \prime}<0$ et l'équation a deux racines réelles distinctes : $y_{3}<y_{4}$.

3. Cas $\mathrm{C}: k^{\prime \prime}=0$ et il y a une racine double $: y_{3}=y_{4}=m$.

On représente dans les figures $9,10,11$ les graphes de $P_{1}$ correspondants aux trois cas. Quand $\varepsilon \neq 0$, on rencontre toujours les trois situations. C'est un phénomène qui ne dépend pas du modèle et qui est associé à la situation géométrique où la trajectoire anormale est stricte, voir le lemme 2.8. Le cas C correspond à la situation où le mouvement de $y(t)$ n'est pas périodique car le temps nécessaire à atteindre la position $m$ devient infini car $P_{1}(y)=-1$ a une racine double. Dans l'espace des phases du pendule, c'est un mouvement localisé sur une séparatrice. Quand $p_{x}=0$, le graphe de $P_{1}$ ne rencontre pas la droite $y=-1$, on peut donc supposer dans les cas B et $\mathrm{C}$ que $p_{x} \neq 0$. Dans le cas $\mathrm{B}, y_{3} y_{4}=\frac{2\left(p_{x}+1\right)}{\lambda}$. Donc si $p_{x} \neq-1$, les 2 racines $y_{3}$ et $y_{4}$ ont le même signe, à savoir le signe de $-\varepsilon p_{x}$. Si $p_{x}=-1, \varepsilon<0$ on a alors $y_{4}=0$ et $y_{3}=\frac{2 \varepsilon}{\lambda}<0$, par ailleurs $\dot{y}(0)=0$ et $\ddot{y}(0)=-\varepsilon>0$. Le système oscille donc entre $y_{4}=0$ et $y_{2}$. Dans le cas C, la racine est $y_{3}=y_{4}=m=-\frac{\varepsilon p_{x}}{\lambda}$ et $m \neq 0$. On peut donc résumer cela dans les lemmes suivants.

Lemme 4.4. Supposons $\varepsilon<0$. Si $k^{\prime \prime}<0$, les racines de $P_{1}(y)=-1$ sont formées de deux points distincts $y_{3}<y_{4}$. Si $p_{x} \neq-1$, elles ont toutes deux le signe de $-\varepsilon p_{x}$. Si $p_{x}=-1$, alors $y_{3}<0$ et $y_{4}=0$. Si $k^{\prime \prime}=0$, la racine est double et est donnée par $m=-\frac{\varepsilon p_{x}}{\lambda}$.

Lemme 4.5. Si $k^{\prime \prime} \neq 0$, une trajectoire de $\dot{y}^{2}+P_{1}^{2}(y)=1$ issue de 0 oscille de façon périodique entre $y_{-}<y_{+}$ où $\left[y_{-}, y_{+}\right]$est l'intervalle $\left[y_{1}, y_{2}\right]$ dans le cas $A$, et l'intervalle $\left[y_{1}, y_{3}\right]$ ou $\left[y_{4}, y_{2}\right]$ contenant 0 dans le cas $B$ si $p_{x} \neq-1$ et l'intervalle $\left[0, y_{2}\right]$ si $p_{x}=-1$. Si $k^{\prime \prime}=0$ le mouvement n'est pas périodique.

Par ailleurs en représentant sur la figure 12 le graphe du potentiel on met en évidence un phénomène dit de doublement de période qui sera explicité en détail ultérieurement.

Enfin, il est important de représenter (Fig. 13) l'ensemble critique $k^{\prime \prime}=0$ et l'on a le lemme suivant.

Lemme 4.6. Supposons $\varepsilon \neq 0$. Alors l'ensemble critique $k^{\prime \prime}=0$ est la trace du graphe $\lambda=\frac{\varepsilon^{2} p_{x}^{2}}{2\left(1+p_{x}\right)}$ dans le domaine $\lambda>0, p_{x} \in[-1,+1]$, voir figure 13 . 


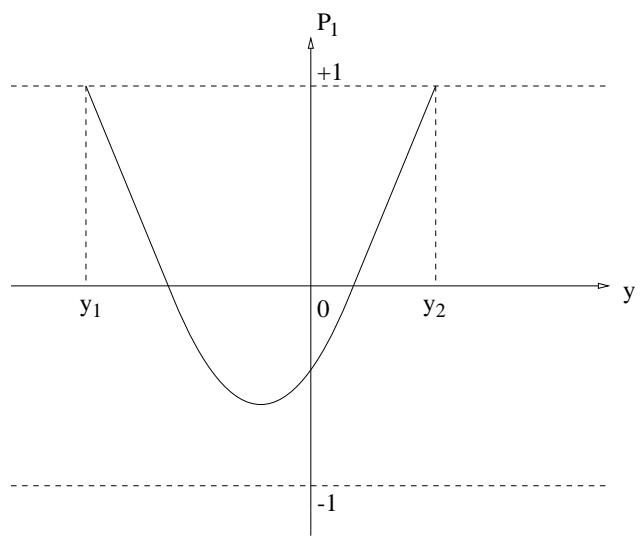

Figure 9. Cas A.

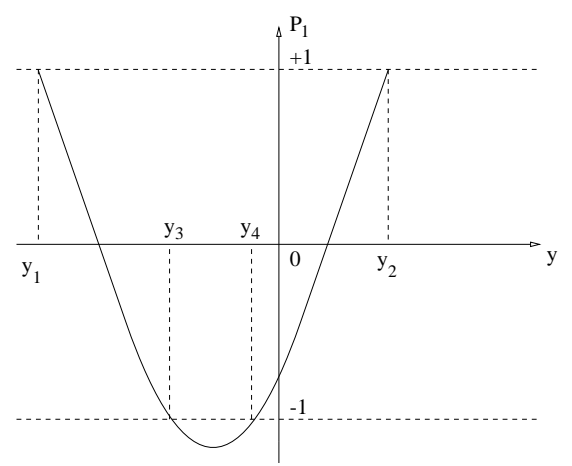

Figure 10. Cas B

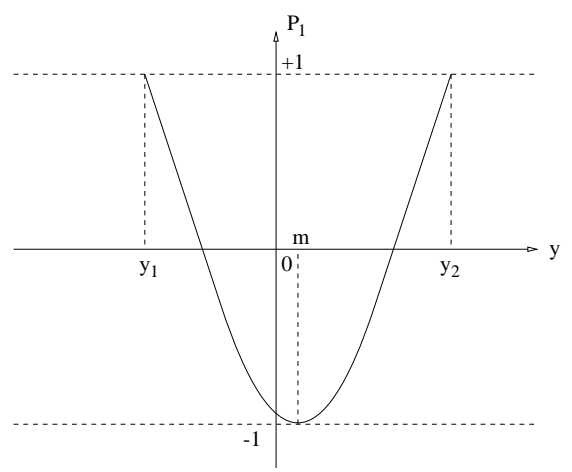

Figure 11. Cas C. 


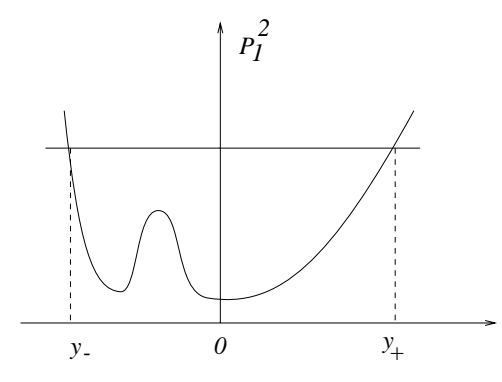

CAS A

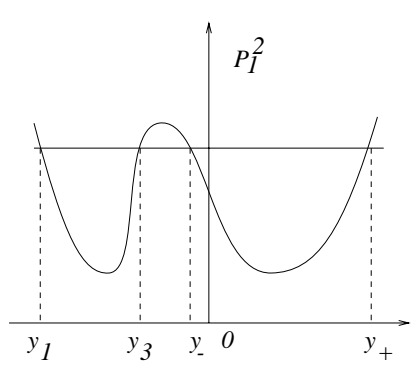

$C A S B$

Figure 12

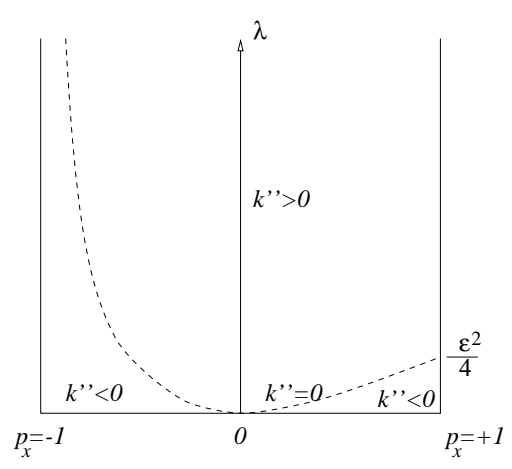

FIGURE 13

\subsection{Description intégrale des géodésiques normales dans les coordonnées normalisées}

Dans le paragraphe 2.6 des formules générales afin de paramétriser les géodésiques normales dans le cas intégrable ont été établies. On va particulariser ces formules dans notre cas et les écrire dans la coordonnée normalisée $\eta$. Ce travail permet ensuite de calculer les développements asymptotiques de la sphère dans la direction anormale.

La géodésique normale de référence est $e:[0, T] \mapsto(x(t), y(t), z(t))$ et dans le cas $\mathrm{A}$ et $\mathrm{B}$, la coordonnée $y$ oscille de façon périodique entre $\left\{y_{-}, y_{+}\right\}$et la période est donnée par :

$$
\mathcal{P}=2 \int_{y_{-}}^{y_{+}} \frac{d y}{\sqrt{1-P_{1}^{2}(y)}}
$$

et dans le cas critique $\mathrm{C}, \mathcal{P}$ devient infinie. Explicitons les formules pour la situation $y=0$. Notons $0<t_{1}<$ $\ldots<t_{N} \leq T$ les temps successifs tels que $y\left(t_{i}\right)=0$ et posons :

$$
\sigma=\left\{\begin{array}{l}
\text { signe } \dot{y}(0) \text { si } \dot{y}(0) \neq 0 \\
\text { signe } \ddot{y}(0) \text { si } \dot{y}(0)=0 .
\end{array}\right.
$$

Observons que l'on ne peut pas avoir $\dot{y}(0)=\ddot{y}(0)=0$, si $\varepsilon<0$, car $\dot{y}(0)=P_{2}(0)=\cos \varphi$ et $\ddot{y}(0)=-\varepsilon P_{1}^{2}(0)$. Donc pour $\varphi \in]-\frac{\pi}{2},+\frac{\pi}{2}[, \dot{y}(0)>0$ et pour $\varphi \in] \frac{\pi}{2}, \frac{3 \pi}{2}\left[, \dot{y}(0)<0\right.$. Les cas restants correspondent à $\varphi \in\left\{-\frac{\pi}{2},+\frac{\pi}{2}\right\}$ où $\dot{y}(0)=0, \ddot{y}(0)>0$ et $y$ garde alors un signe constant. 
Pour calculer les géodésiques, on doit distinguer les cas $\sigma=+1$ et $\sigma=-1$. Si $y(T)=0$ avec $T=t_{N}$ on obtient les formules suivantes :

- $\underline{N=2 p+1}$ :

$$
\begin{aligned}
& x(T)=2 \int_{0}^{y_{\sigma}} \frac{\sigma(1+\varepsilon y) P_{1}(y) d y}{\sqrt{1-P_{1}^{2}(y)}}+(N-1) \int_{y-}^{y_{+}} \frac{(1+\varepsilon y) P_{1}(y) d y}{\sqrt{1-P_{1}^{2}(y)}} \\
& z(T)=\int_{0}^{y_{\sigma}} \frac{\sigma y^{2} P_{1}(y) d y}{\sqrt{1-P_{1}^{2}(y)}}+(N-1) \int_{y-}^{y_{+}} \frac{y^{2} P_{1}(y) d y}{2 \sqrt{1-P_{1}^{2}(y)}} \cdot
\end{aligned}
$$

- $\underline{N=2 p}$ :

$$
\begin{aligned}
& x(T)=N \int_{y_{-}}^{y_{+}} \frac{(1+\varepsilon y) P_{1}(y) d y}{\sqrt{1-P_{1}^{2}(y)}} \\
& z(T)=N \int_{y_{-}}^{y_{+}} \frac{y^{2} P_{1}(y) d y}{2 \sqrt{1-P_{1}^{2}(y)}} .
\end{aligned}
$$

Avec :

$$
\eta=\frac{1}{2 k}\left(y \sqrt{\lambda}+\frac{\varepsilon p_{x}}{\sqrt{\lambda}}\right), \quad \eta_{\sigma}=\sigma
$$

on obtient

$$
\begin{aligned}
\eta(0) & =\frac{\varepsilon p_{x}}{2 k \sqrt{\lambda}} \\
y & =\frac{1}{\sqrt{\lambda}}\left(2 k \eta-\frac{\varepsilon p_{x}}{\sqrt{\lambda}}\right) \\
1-P_{1}^{2}(y) & =4 k^{2}\left(1-\eta^{2}\right)\left(k^{\prime \prime}+k^{2} \eta^{2}\right) \\
P_{1}(y) & =2 k^{2} \eta^{2}+p_{x}-\frac{\varepsilon^{2} p_{x}^{2}}{2 \lambda} .
\end{aligned}
$$

Introduisons alors :

$$
\begin{aligned}
X^{\sigma} & =2 \int_{0}^{y_{\sigma}} \frac{\sigma(1+\varepsilon y) P_{1}(y) d y}{\sqrt{1-P_{1}^{2}(y)}} \\
Z^{\sigma} & =\int_{0}^{y_{\sigma}} \frac{\sigma y^{2} P_{1}(y) d y}{\sqrt{1-P_{1}^{2}(y)}}
\end{aligned}
$$

et

$$
\theta^{\sigma}=2 \int_{0}^{y_{\sigma}} \frac{\sigma d \eta}{\sqrt{1-P_{1}^{2}(y)}}
$$


où $X^{\sigma}, Z^{\sigma}$ représentent respectivement la dérive de $x$ et $z$ après la première intersection avec le plan $y=0$ et $\theta^{\sigma}$ la longueur de la trajectoire correspondante. Il vient les formules

$$
\begin{aligned}
X^{\sigma} & =\frac{2}{\sqrt{\lambda}} \int_{\eta(0)}^{\eta_{\sigma}} \frac{\sigma\left(1+\frac{2 \varepsilon k \eta}{\sqrt{\lambda}}-\frac{\varepsilon^{2} p_{x}}{\lambda}\right)\left(2 k^{2} \eta^{2}+p_{x}-\frac{\varepsilon^{2} p_{x}^{2}}{2 \lambda}\right) d \eta}{\sqrt{\left(1-\eta^{2}\right)\left(k^{\prime \prime}+k^{2} \eta^{2}\right)}} \\
Z^{\sigma} & =\frac{1}{\lambda^{\frac{3}{2}}} \int_{\eta(0)}^{\eta_{\sigma}} \frac{\sigma\left(2 k \eta-\frac{\varepsilon p_{x}}{\lambda}\right)^{2}\left(2 k^{2} \eta^{2}+p_{x}-\frac{\varepsilon^{2} p_{x}^{2}}{2 \lambda}\right) d \eta}{\sqrt{\left(1-\eta^{2}\right)\left(k^{\prime \prime}+k^{2} \eta^{2}\right)}} \\
\theta^{\sigma} & =\frac{2}{\sqrt{\lambda}} \int_{\eta(0)}^{\eta_{\sigma}} \frac{\sigma d \eta}{\sqrt{\left(1-\eta^{2}\right)\left(k^{\prime \prime}+k^{2} \eta^{2}\right)}} .
\end{aligned}
$$

\subsection{Cas A : Paramétrisation des géodésiques en utilisant les fonctions de Jacobi}

\subsubsection{Paramétrisation}

Afin de calculer les géodésiques, nous intègrons l'équation sous forme normale

$$
\frac{\dot{\eta}^{2}}{\lambda}=\left(1-\eta^{2}\right)\left(k^{\prime \prime}+k^{2} \eta^{2}\right), \quad k^{\prime \prime}>0
$$

où la variable $\eta$ oscille entre -1 et +1 avec une période $\mathcal{P}$ donnée par :

$$
\mathcal{P}=\frac{2}{\sqrt{\lambda}} \int_{-1}^{+1} \frac{d \eta}{\sqrt{\left(1-\eta^{2}\right)\left(k^{\prime \prime}+k^{2} \eta^{2}\right)}}
$$

et $\mathcal{P}$ est relié à $K(k)$ intégrale elliptique complète de première espèce par la formule : $\mathcal{P}=\frac{4}{\sqrt{\lambda}} K(k)$. Pour intégrer l'équation caractéristique on distingue entre le cas $\sigma=+1$ et $\sigma=-1$. En effet pour $\sigma=+1$, on intègre avec la branche

$$
\frac{d \eta}{d s}=\sqrt{\left(1-\eta^{2}\right)\left(k^{\prime \prime}+k^{2} \eta^{2}\right)}
$$

où $s=t \sqrt{\lambda}$ et $t$ est assez petit. On obtient donc :

$$
-s+\int_{\eta(0)}^{1} \frac{d \eta}{\sqrt{\left(1-\eta^{2}\right)\left(k^{\prime \prime}+k^{2} \eta^{2}\right)}}=\int_{\eta(s)}^{1} \frac{d \eta}{\sqrt{\left(1-\eta^{2}\right)\left(k^{\prime \prime}+k^{2} \eta^{2}\right)}}
$$

et donc

$$
\eta(s)=\operatorname{cn}\left(T_{1}-s, k\right)
$$

où $T_{1}=\int_{\eta(0)}^{1} \frac{d \eta}{\sqrt{\left(1-\eta^{2}\right)\left(k^{\prime \prime}+k^{2} \eta^{2}\right)}}$. En utilisant la relation :

$$
\operatorname{cn}\left(T_{1}-s\right)=\operatorname{cn}\left(2 K-\left(s+2 K-T_{1}\right)\right)=-\operatorname{cn}\left(s+2 K-T_{1}\right),
$$

on obtient :

$$
\eta(s)=-\operatorname{cn}\left(s+2 K-T_{1}, k\right)
$$

Quand $\sigma=-1$, on utilise la branche

$$
\frac{d \eta}{d s}=-\sqrt{\left(1-\eta^{2}\right)\left(k^{\prime \prime}+k^{2} \eta^{2}\right)}
$$


donc

$$
s+\int_{\eta(0)}^{1} \frac{d \eta}{\sqrt{\left(1-\eta^{2}\right)\left(k^{\prime \prime}+k^{2} \eta^{2}\right)}}=\int_{\eta(s)}^{1} \frac{d \eta}{\sqrt{\left(1-\eta^{2}\right)\left(k^{\prime \prime}+k^{2} \eta^{2}\right)}}
$$

d'où

$$
s+T_{1}=\mathrm{cn}^{-1}(\eta(s), k)
$$

où $\mathrm{cn}^{-1}$ est la fonction réciproque de $\mathrm{cn}$. Donc dans les deux cas on peut écrire :

$$
\eta(t)=-\sigma \operatorname{cn}(\phi+t \sqrt{\lambda}, k)
$$

où $\phi=T_{1}$ si $\sigma=-1$ et $\phi=2 K-T_{1}$ si $\sigma>0$. Ce calcul est valide pour tout $t$, par analycité, dans le domaine $U$. Comme on a

$$
y=\frac{1}{\sqrt{\lambda}}\left(2 k \eta-\frac{\varepsilon p_{x}}{\sqrt{\lambda}}\right)
$$

il vient le lemme suivant.

Lemme 4.7. Si $k^{\prime \prime}>0$, le mouvement de y est donné par :

$$
y(t)=-\sigma \frac{2 k}{\sqrt{\lambda}} \operatorname{cn}(\phi+t \sqrt{\lambda}, k)-\frac{\varepsilon \sin \varphi}{\lambda} .
$$

$C^{\prime}$ 'est un mouvement périodique en $s=t \sqrt{\lambda}$ de période $4 K(k)$ et d'amplitude $\frac{2 k}{\sqrt{\lambda}}$. Le paramètre $\varepsilon$ induit un déphasage et une translation, par rapport au cas plat.

Le calcul des deux autres composantes $x, z$ de la géodésique est laborieux et requiert l'utilisation du formulaire de [21] concernant les fonctions elliptiques. En effet :

$$
\dot{x}=p_{x}+2 \varepsilon p_{x} y+\left(\varepsilon^{2} p_{x}+\frac{\lambda}{2}\right) y^{2}+\frac{\varepsilon \lambda}{2} y^{3}
$$

et

$$
\begin{aligned}
& \int_{0}^{t} y(s) d s=-\frac{2 \sigma k}{\sqrt{\lambda}} \int_{0}^{t} \operatorname{cn}(\phi+s \sqrt{\lambda}) d s-\frac{\varepsilon p_{x} t}{\lambda}=-\frac{2 \sigma k}{\lambda} \int_{\phi}^{\phi+t \sqrt{\lambda}} \operatorname{cn} u d u-\frac{\varepsilon p_{x} t}{\lambda}, \\
& \int_{0}^{t} y^{2}(s) d s=\frac{4 k^{2}}{\lambda^{\frac{3}{2}}} \int_{\phi}^{\phi+t \sqrt{\lambda}} \operatorname{cn}^{2} u d u+\frac{4 \sigma \varepsilon p_{x} k}{\lambda^{2}} \int_{\phi}^{\phi+t \sqrt{\lambda}} \operatorname{cn} u d u+\frac{\varepsilon^{2} p_{x}^{2} t}{\lambda^{2}}, \\
& \int_{0}^{t} y^{3}(s) d s=-\frac{8 \sigma k^{3}}{\lambda^{2}} \int_{\phi}^{\phi+t \sqrt{\lambda}} \operatorname{cn}^{3} u d u-\frac{12 \varepsilon p_{x} k^{2}}{\lambda^{\frac{5}{2}}} \int_{\phi}^{\phi+t \sqrt{\lambda}} \operatorname{cn}^{2} u d u-\frac{6 \sigma \varepsilon^{2} p_{x}^{2} k}{\lambda^{3}} \int_{\phi}^{\phi+t \sqrt{\lambda}} \operatorname{cn} u d u-\frac{\varepsilon^{3} p_{x}^{3} t}{\lambda^{3}}
\end{aligned}
$$

et l'on obtient donc :

$$
\begin{aligned}
x(t)= & t p_{x}\left(1-\frac{3 \varepsilon^{2} p_{x}}{2 \lambda}+\frac{\varepsilon^{4} p_{x}^{2}}{2 \lambda^{2}}\right)+\frac{\sigma \varepsilon p_{x} k}{\lambda}\left(\frac{\varepsilon^{2} p_{x}}{\lambda}-2\right) \int_{\phi}^{\phi+t \sqrt{\lambda}} \operatorname{cn} u d u \\
& +\frac{2 k^{2}}{\sqrt{\lambda}}\left(1-\frac{\varepsilon^{2} p_{x}}{\lambda}\right) \int_{\phi}^{\phi+t \sqrt{\lambda}} \operatorname{cn}^{2} u d u-\frac{4 \sigma \varepsilon k^{3}}{\lambda} \int_{\phi}^{\phi+t \sqrt{\lambda}} \operatorname{cn}^{3} u d u
\end{aligned}
$$


que l'on évalue ensuite en utilisant les relations de [21], p. 40, 62 et 87 :

$$
\begin{aligned}
\int_{\phi}^{\phi+t \sqrt{\lambda}} \operatorname{cn} u d u= & \frac{1}{k}[\arcsin (k \operatorname{sn}(\phi+t \sqrt{\lambda})-\arcsin (k \operatorname{sn} \phi)] \\
k^{2} \int_{\phi}^{\phi+t \sqrt{\lambda}} \operatorname{cn}^{2} u d u= & E(\phi+t \sqrt{\lambda})-E(\phi)-k^{\prime \prime} t \sqrt{\lambda} \\
\int_{\phi}^{\phi+t \sqrt{\lambda}} \operatorname{cn}^{3} u d u= & \frac{1}{2 k^{3}}\left[\left(2 k^{2}-1\right)(\arcsin (k \operatorname{sn}(\phi+t \sqrt{\lambda}))-\arcsin (k \operatorname{sn} \phi))\right. \\
& +k(\operatorname{sn}(\phi+t \sqrt{\lambda}) \operatorname{dn}(\phi+t \sqrt{\lambda})-\operatorname{sn} \phi \operatorname{dn} \phi)]
\end{aligned}
$$

De même :

$$
\dot{z}=\frac{p_{x}}{2} y^{2}+\frac{\varepsilon p_{x}}{2} y^{3}+\frac{\lambda}{4} y^{4}
$$

qui s'évalue à l'aide des formules (4.18) et de la relation :

$$
\begin{aligned}
\int_{\phi}^{\phi+t \sqrt{\lambda}} \operatorname{cn}^{4} u d u= & \frac{1}{3 k^{4}}\left[\left(2-3 k^{2}\right) k^{\prime \prime} t \sqrt{\lambda}+2\left(2 k^{2}-1\right)(E(\phi+t \sqrt{\lambda})-E(\phi))\right. \\
& \left.+k^{2}(\operatorname{sn}(\phi+t \sqrt{\lambda}) \operatorname{cn}(\phi+t \sqrt{\lambda}) \operatorname{dn}(\phi+t \sqrt{\lambda})-\operatorname{sn} \phi \operatorname{cn} \phi \operatorname{dn} \phi)\right]
\end{aligned}
$$

et après simplification, on obtient finalement le résultat suivant.

Proposition 4.8. Les géodésiques normales issues de 0 et correspondant $\grave{a} \lambda>0, k^{\prime \prime}>0$ dans $U$ sont les restrictions à $U$ des courbes suivantes:

$$
\begin{aligned}
x(t)= & {\left[-t+\frac{2}{\sqrt{\lambda}}(E(u)-E(\phi))\right]\left(1-\frac{\varepsilon^{2} \sin \varphi}{\lambda}\right)-\frac{2 \sigma \varepsilon k}{\lambda}(\operatorname{sn} u \operatorname{dn} u-\operatorname{sn} \phi \operatorname{dn} \phi) } \\
y(t)= & -\frac{\sigma 2 k}{\sqrt{\lambda}} \operatorname{cn} u-\frac{\varepsilon \sin \varphi}{\lambda} \\
z(t)= & t\left(\frac{1+\sin \varphi}{3 \lambda}-\frac{2 \varepsilon^{2} \sin ^{2} \varphi}{3 \lambda^{2}}\right)+\frac{2 \sin \varphi}{3 \lambda^{\frac{3}{2}}}\left(\frac{2 \varepsilon^{2} \sin \varphi}{\lambda}-1\right)(E(u)-E(\phi)) \\
& +\frac{2 \sigma \varepsilon \sin \varphi k}{\lambda^{2}}(\operatorname{sn} u \operatorname{dn} u-\operatorname{sn} \phi \operatorname{dn} \phi)+\frac{4 k^{2}}{3 \lambda^{\frac{3}{2}}}(\operatorname{snn} u \operatorname{cn} u \operatorname{dn} u-\operatorname{sn} \phi \operatorname{cn} \phi \operatorname{dn} \phi)
\end{aligned}
$$

ò̀ $u=\phi+t \sqrt{\lambda}, \phi=T_{1}$ si $\sigma<0, \phi=2 K-T_{1}$ si $\sigma>0$ et $T_{1}=\int_{\eta(0)}^{1} \frac{d \eta}{\sqrt{\left(1-\eta^{2}\right)\left(k^{\prime \prime}+k^{2} \eta^{2}\right)}}, \eta(0)=\frac{\varepsilon \sin \varphi}{2 k \sqrt{\lambda}}$.

\subsubsection{Classification}

Une géodésique normale $e:[0, T] \rightarrow U$ est une courbe paramétrisée par le paramètre $s=t \sqrt{\lambda}$. Sa projection dans le plan $(\theta, \dot{\theta})$ correspond à la trajectoire d'un pendule oscillant. Sa projection dans le plan $(x, y)$ est plus complexe. Dans le cas plat $\varepsilon=0$, c'est un élastique avec point d'inflexion, voir [28], p. 402. Dans le cas $\varepsilon \neq 0$ c'est une courbe plus compliquée. On représente sur la figure 14, les formes typiques de ces courbes (la borne $|y \varepsilon|<1$ n'étant pas prise en compte). 


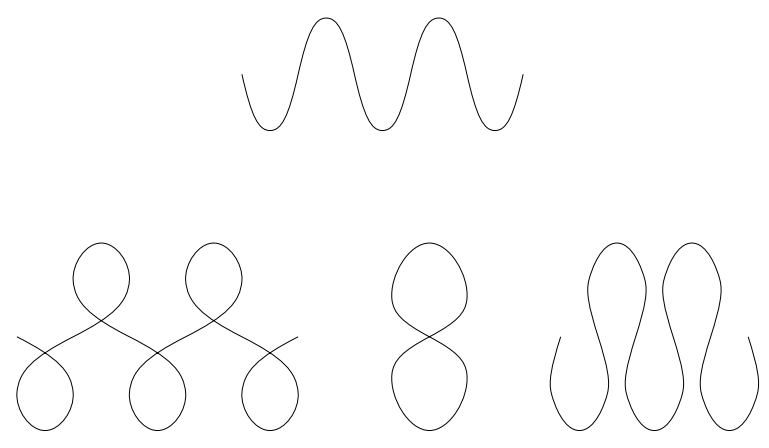

FIGURE 14

\subsection{Cas B : Paramétrisation des géodésiques en utilisant les fonctions de Jacobi}

\subsubsection{Paramétrisation}

Dans ce paragraphe, nous allons traiter la situation $k^{\prime \prime}<0$. On a :

$$
2 k^{2}=1-p_{x}+\frac{\varepsilon^{2} p_{x}^{2}}{2 \lambda}>0 \text { si } \varepsilon \neq 0,2 k^{\prime \prime}=1+p_{x}-\frac{\varepsilon^{2} p_{x}^{2}}{2 \lambda}
$$

et $k^{\prime \prime}+k^{2}=1$. On introduit :

$$
\hat{k}^{\prime 2}=-\frac{k^{\prime \prime}}{k^{2}}=1-\frac{1}{k^{2}}
$$

et $0<\hat{k}^{\prime 2}<1$. Soient donc $0<\hat{k}, \hat{k}^{\prime}<1$ définis par $\hat{k}^{2}+\hat{k}^{\prime 2}=1$.

L'équation caractéristique s'écrit dans le cas B :

$$
\frac{\dot{\eta}^{2}}{k^{2} \lambda}=\left(1-\eta^{2}\right)\left(\eta^{2}-\hat{k}^{\prime 2}\right)
$$

D'après les lemmes 4.3 et 4.4 , si $p_{x}<0$ la coordonnée $y$ oscille entre $y_{2}$ et $y_{4}$ avec $y_{2}>0 \geq y_{4}$ et $\eta$ oscille entre $\hat{k}^{\prime}$ et 1 . Si $p_{x}>0$, alors $y$ oscille entre $y_{1}$ et $y_{3}$ où $y_{1}<0 \leq y_{3}$ et $\eta$ oscille entre -1 et $-\hat{k}^{\prime}$. Dans les deux cas il faut distinguer entre $\sigma=+1$ et $\sigma=-1$. Finalement, on obtient comme dans le cas A la paramétrisation :

$$
\eta(t)=\mu \operatorname{dn}(\psi+t k \sqrt{\lambda}, \hat{k})
$$

où $\mu=-\operatorname{sign}\left(p_{x}\right)$ et $\psi$ est défini par $\psi=T_{2}$ si $\mu \sigma<0$ et $\psi=-T_{2}$ si $\mu \sigma>0$ où $T_{2}=\int_{|\eta(0)|}^{1} \frac{d \eta}{\sqrt{\left(1-\eta^{2}\right)\left(\eta^{2}-\hat{k}^{\prime 2}\right)}}$ et $\eta(0)=\frac{\varepsilon p_{x}}{2 k \sqrt{\lambda}}$. On obtient le lemme suivant.

Lemme 4.9. Si $k^{\prime \prime}<0$, le mouvement de y est donné par :

$$
y(t)=\mu \frac{2 k}{\sqrt{\lambda}} \operatorname{dn}(\psi+t k \sqrt{\lambda})-\frac{\varepsilon \sin \varphi}{\lambda} .
$$

$C^{\prime}$ est un mouvement périodique en $s^{\prime}=t k \sqrt{\lambda}$, de période $2 K(\hat{k})$ et d'amplitude $\frac{k(1-\hat{k})}{\sqrt{\lambda}}$ et il est relié à la vitesse angulaire d'un pendule en mouvement de rotation. 


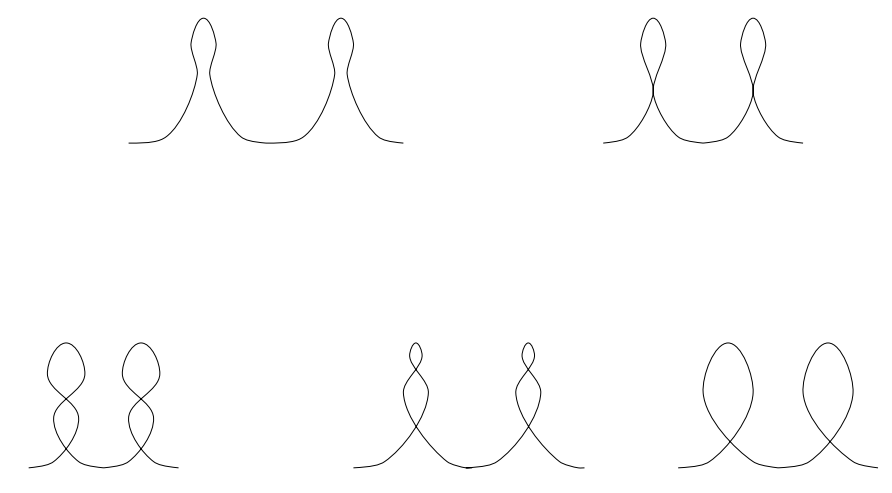

FIGURE 15

Comme dans le cas A, on peut calculer $x(t), z(t)$ en utilisant le formulaire de [21]. On obtient la proposition suivante.

Proposition 4.10. Les géodésiques normales issues de 0 , correspondant $\grave{a} \lambda>0, k^{\prime \prime}<0$ et contenues dans $U$ sont les restrictions à $U$ des courbes :

$$
\begin{aligned}
x(t)= & t \sin \varphi\left(1-\frac{3 \varepsilon^{2} \sin \varphi}{2 \lambda}+\frac{\varepsilon^{4} \sin ^{2} \varphi}{2 \lambda^{2}}\right)+\frac{2 k}{\sqrt{\lambda}}\left(1-\frac{\varepsilon^{2} \sin \varphi}{\lambda}\right)(E(u)-E(\psi))+\mu \frac{2 \varepsilon}{\lambda}(\operatorname{sn} u \operatorname{cn} u-\operatorname{sn} \psi \operatorname{cn} \psi) \\
y(t)= & \mu \frac{2 k}{\sqrt{\lambda}} \operatorname{dn} u-\frac{\varepsilon \sin \varphi}{\lambda} \\
z(t)= & \frac{t}{3 \lambda}\left(1-\sin ^{2} \varphi+\frac{5 \varepsilon^{2} \sin ^{3} \varphi}{2 \lambda}-\frac{\varepsilon^{4} \sin ^{4} \varphi}{\lambda^{2}}\right)+\frac{2 \sin \varphi k}{3 \lambda^{\frac{3}{2}}}\left(\frac{2 \varepsilon^{2} \sin \varphi}{\lambda}-1\right)(E(u) \\
& -E(\psi))-\mu \frac{2 \varepsilon \sin \varphi}{\lambda^{2}}(\operatorname{sn} u \operatorname{cn} u-\operatorname{sn} \psi \operatorname{cn} \psi)+\frac{4 k}{3 \lambda^{\frac{3}{2}}}(\operatorname{sn} u \operatorname{cn} u \operatorname{dn} u-\operatorname{sn} \psi \operatorname{cn} \psi \operatorname{dn} \psi)
\end{aligned}
$$

ò̀ $\mu=-\operatorname{sign}\left(p_{x}\right), u=\psi+t k \sqrt{\lambda}, \operatorname{sn} u, \operatorname{cn} u, \operatorname{dn} u, E(u)$ sont les fonctions de Jacobi de module $\hat{k}$ et $\psi=T_{2}$ si $\mu \sigma<0, \psi=-T_{2}$ si $\mu \sigma>0$ avec $T_{2}=\int_{|\eta(0)|}^{1} \frac{d \eta}{\sqrt{\left(1-\eta^{2}\right)\left(\eta^{2}-\hat{k}^{\prime 2}\right)}}, \eta(0)=\frac{\varepsilon \sin \varphi}{2 k \sqrt{\lambda}}$.

\subsubsection{Classification}

Lorsque nous sommes dans la situation $k^{\prime \prime}<0$, une géodésique normale se paramétrise par $s^{\prime}=t k \sqrt{\lambda}$. Sa projection dans le plan $(\theta, \dot{\theta})$ correspond à la trajectoire d'un pendule en rotation. Sa projection dans le plan $(x, y)$ présente une analogie avec un élastique sans point d'inflexion, [28], p. 402. On a représenté sur la figure 15 des courbes typiques (la contrainte $|y \varepsilon|<1$ n'est pas prise en compte).

\subsection{Le phénomène de doublement de période et l'instabilité en résultant}

Quand le module de la fonction de Jacobi cn ou dn est fixé à $k$, on observe que la période de cn est de $4 K$ et celle de dn est de $2 K$, donc la période de cn est double. De plus, on a la relation :

$$
\operatorname{dn}^{2} s=k^{\prime 2}+k^{2} \mathrm{cn}^{2} s
$$




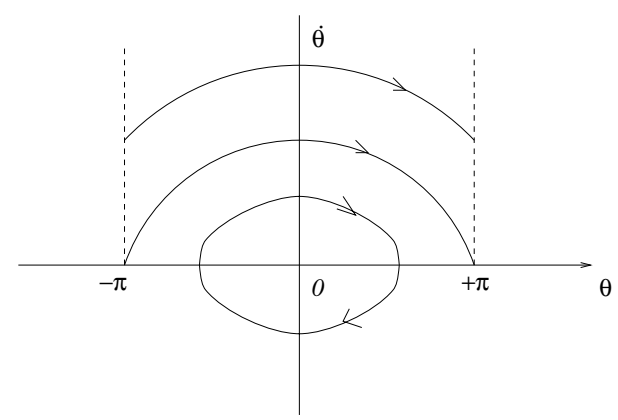

FiguRE 16

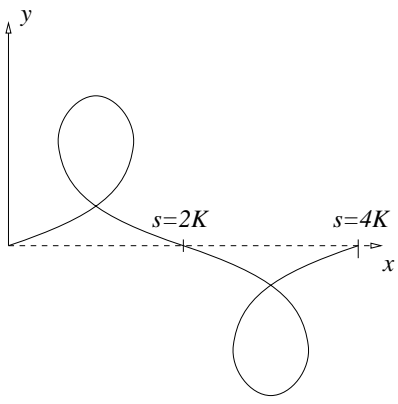

Inflexionnel

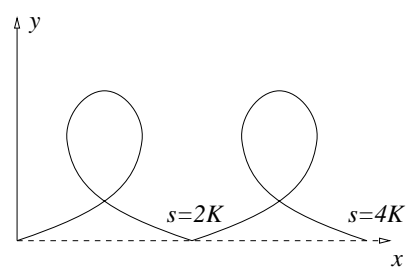

Non inflexionnel

FiguRE 17

où $k^{\prime 2}=1-k^{2} \rightarrow 0$ quand $k \rightarrow 1$ et $\operatorname{dn} \sim \mid$ cn $\mid$ quand $k \rightarrow 1$. Ce résultat indique que dans le développement formel du paragraphe 2.5 :

$$
y(t, \lambda, \varphi)=\frac{1}{\sqrt{\lambda}} Y_{0}(t \sqrt{\lambda}, 1, \varphi)+\frac{1}{\lambda} Y_{1}(t \sqrt{\lambda}, 1, \varphi)+\ldots
$$

le terme $\frac{1}{\sqrt{\lambda}} Y_{0}$ du cas plat ne peut représenter $y(t)$ quand $k \rightarrow 1, k^{\prime \prime}<0$ que pour une longueur $|t \sqrt{\lambda}| \leq 2 K$.

C'est clair aussi dans la projection des géodésiques dans le plan des phases $(\theta, \dot{\theta})$ sur le cylindre et dans la projection dans le plan $(x, y)$, voir figures 16 et 17 . Ces deux figures nous indiquent géométriquement que l'on ne peut approximer dans la topologie $C^{0}$ une géodésique correspondant à $k^{\prime \prime}<0$ par une géodésique correspondant à $k^{\prime \prime}>0$ au voisinage de $k^{\prime \prime}=0$ que pour une longueur $t \sqrt{\lambda} \leq 2 K$.

\subsection{Paramétrisation des géodésiques dans le cas $\mathbf{C}$}

Dans le cas $k^{\prime \prime}=0$, on utilise les fonctions hyperboliques pour calculer les géodésiques. En effet en calculant il vient :

$$
y(t)=\mu \frac{2}{\sqrt{\lambda}} \operatorname{sech}(\Omega+t \sqrt{\lambda})-\frac{\varepsilon \sin \varphi}{\lambda}
$$



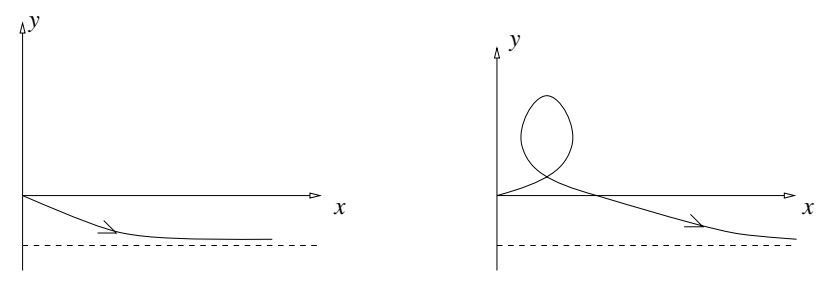

FIGURE 18

où $\lambda, \varphi$ sont liés par $k^{\prime \prime}=0$, c'est-à-dire

$$
\lambda=\frac{\varepsilon^{2} \sin ^{2} \varphi}{2(1+\sin \varphi)}
$$

et $\mu=-\operatorname{sign}(\sin \varphi), \Omega=T_{3}$ si $\mu \sigma<0$ et $\Omega=-T_{3}$ si $\mu \sigma>0$ où

$$
\Omega=\int_{|\eta(0)|}^{1} \frac{d \eta}{\sqrt{\eta^{2}\left(1-\eta^{2}\right)}}, \quad \eta(0)=\mu \sqrt{\frac{1+\sin \varphi}{2}}
$$

et par définition sech $=\frac{1}{\cosh }$. En calculant les autres coordonnées nous obtenons la proposition suivante.

Proposition 4.11. Les géodésiques normales issues de 0 correspondant à $\lambda>0, k^{\prime \prime}=0$ dans le domaine $U$ sont les restrictions à $U$ des courbes :

$$
\begin{aligned}
x(t)= & t\left(1+\frac{2}{\sin \varphi}\right)+\frac{2}{\sqrt{\lambda}}\left(1-\frac{\varepsilon^{2} \sin \varphi}{\lambda}\right)(\tanh u-\tanh \Omega) \\
& +\frac{2 \mu \varepsilon}{\lambda}(\tanh u \operatorname{sech} u-\tanh \Omega \operatorname{sech} \Omega) \\
y(t)= & \mu \frac{2}{\sqrt{\lambda}} \operatorname{sech} u-\frac{\varepsilon \sin \varphi}{\lambda} \\
z(t)= & -t\left(1+\frac{\sin \varphi}{\lambda}\right)+\frac{2 \sin \varphi}{3 \lambda^{\frac{3}{2}}}\left(\frac{2 \varepsilon^{2} \sin \varphi}{\lambda}-1\right)(\tanh u-\tanh \Omega) \\
& -\frac{2 \mu \varepsilon \sin \varphi}{\lambda^{2}}(\tanh u \operatorname{sech} u-\tanh \Omega \operatorname{sech} \Omega)+\frac{4}{3 \lambda^{\frac{3}{2}}}\left(\tanh u \operatorname{sech}^{2} u-\tanh \Omega \operatorname{sech}^{2} \Omega\right)
\end{aligned}
$$

avec $u=\Omega+t \sqrt{\lambda}, \lambda=\frac{\varepsilon^{2} \sin ^{2} \varphi}{2(1+\sin \varphi)}$ et $\Omega=T_{3}$ si $\mu \sigma<0, \Omega=-T_{3}$ si $\mu \sigma>0$ et $T_{3}=\int_{|\eta(0)|}^{1} \frac{d \eta}{\sqrt{\eta^{2}\left(1-\eta^{2}\right)}}$, $\eta(0)=\mu \sqrt{\frac{1+\sin \varphi}{2}}$.

\subsubsection{Représentation des solutions}

Dans le cas C, le mouvement de $y$ n'est pas périodique. Dans le plan $(\theta, \dot{\theta})$, la géodésique évolue sur la séparatrice. Les courbes paramétrisées par $s=t \sqrt{\lambda}$ et projetées dans le plan $(x, y)$ représentent la transition entre un élastique à point d'inflexion et un élastique sans point d'inflexion. On représente les deux caractéristiques importantes d'une telle courbe sur la figure 18. 


\subsection{Paramétrisation dans le cas $\mathbf{D}: \lambda=0$}

Si $\lambda=0, y$ est solution de l'équation linéaire :

$$
\ddot{y}+\varepsilon \sin ^{2} \varphi(1+\varepsilon y)=0, y(0)=0, \dot{y}(0)=\cos \varphi .
$$

Dans le cas où $\sin \varphi=0, y= \pm t$ et la géodésique est la droite $t \rightarrow(0, \pm t, 0)$. Si $\sin \varphi \neq 0$, la solution $y$ est donnée par :

$$
y(t)=A \cos (\beta t+\psi)-\frac{1}{\varepsilon}
$$

où $\beta=\varepsilon \sin \varphi, \psi=-\arctan (\cot \varphi)$. On obtient les autres composantes par intégration élémentaires, d'où la paramétrisation suivante.

Proposition 4.12. Les géodésiques normales issues de 0, associées à $\lambda=0$ dans le domaine $U$ sont les restrictions à $U$ des courbes :

$$
\begin{aligned}
x(t)= & \frac{1}{4 \varepsilon \cos ^{2} \psi}[2 \beta t+\sin (2(\psi+\beta t))-\sin 2 \psi] \\
y(t)= & \frac{1}{\varepsilon \cos \varphi} \cos (\psi+\beta t)-\frac{1}{\varepsilon} \\
z(t)= & \frac{1}{24 \varepsilon^{3} \cos \psi}\left[-\frac{12 \beta t}{\cos \psi}+12 \sin (\psi+\beta t)+\frac{9 \sin (\psi+\beta t)}{\cos ^{2} \psi}-\frac{6 \sin (2(\psi+\beta t))}{\cos \psi}\right. \\
& \left.+\frac{\sin (3(\psi+\beta t))}{\cos ^{2} \psi}-12 \sin \psi-\frac{9 \sin \psi}{\cos ^{2} \psi}+\frac{6 \sin 2 \psi}{\cos \psi}-\frac{\sin 3 \psi}{\cos ^{2} \psi}\right]
\end{aligned}
$$

si $\sin \varphi \neq 0$, avec $\beta=\varepsilon \sin \psi, \psi=-\arctan (\cot \varphi) \in]-\frac{\pi}{2}, \frac{\pi}{2}[$ et la droite $t \mapsto(0, \pm t, 0)$ si $\sin \varphi=0$.

\subsection{Intersection du front d'onde de petit rayon avec le plan de Martinet $y=0$}

\subsubsection{Préliminaires}

Notre paramétrisation explicite des géodésiques à l'aide des fonctions de Jacobi nous permet à priori de calculer le lieu conjugué et de coupure, comme dans le cas plat et de représenter la sphère. Ces calculs sont complexes car il faut résoudre des équations non triviales dans la catégorie des fonctions de Jacobi. Par ailleurs les propriétés intéressantes de la sphère sont localisées au voisinage de la direction anormale. On va donc étudier la trace de la sphère et du front d'onde sur le plan de Martinet $y=0$, ce travail pouvant être généralisé à tout autre plan, en particulier à la famille des plans contenant la direction anormale, ceci pour représenter la sphère dans son intégralité.

L'étude contient clairement deux parties. La première est de représenter les extrémités des géodésiques de longueur $r$ en utilisant leur paramétrisation explicite à l'aide des fonctions de Jacobi et leur maniement en utilisant le package sur les fonctions elliptiques sous Mathematica. Ce travail donne des résultats satisfaisants excepté au voisinage de la direction anormale, du fait de la singularité logarithmique de l'application exponentielle. On peut néanmoins observer que la catégorie exp-log de la singularité garantit de bonnes extrapolations car l'on représente des courbes peu oscillantes. Si l'on veut avoir une représentation précise des solutions au voisinage de la direction anormale, on doit faire des développements asymptotiques.

\subsubsection{Application retour}

Une géodésique normale définie sur $[0, T]$ issue de 0 , paramétrisée par la longueur d'arc et associée à $p_{x}, p_{y}(0)$, $p_{z}$ où l'on a posé $p_{x}=\sin \varphi, p_{y}(0)=\cos \varphi, p_{z}=\lambda$, est dénotée

$$
e(t, \varphi, \lambda)=(x(t, \varphi, \lambda), y(t, \varphi, \lambda), z(t, \varphi, \lambda)) .
$$


Par symétrie on peut supposer $\lambda \geq 0$ et par ailleurs on peut imposer $\varepsilon<0$. Si $y(t, \varphi, \lambda)$ est non identiquement nul, on note $0<t_{1}(\varphi, \lambda)<\ldots<t_{n}(\varphi, \lambda) \leq T$ les temps successifs tels que $y\left(t_{i}\right)=0$. La suite $X_{n}(\varphi, \lambda), Z_{n}(\varphi, \lambda)$ est définie par $e\left(t_{n}(\varphi, \lambda), \varphi, \lambda\right)=\left(X_{n}(\varphi, \lambda), 0, Z_{n}(\varphi, \lambda)\right)$.

L'application retour est définie comme suit. Soit $R_{1}$ l'application dont le domaine $D_{1}$ est un sous ensemble du cylindre $\{(\varphi, \lambda), \lambda \geq 0\}$ et qui associe à $(\varphi, \lambda)$ la première intersection (non nulle) avec le plan $y=0$, $R_{1}: D_{1} \mapsto\left(X_{1}, Z_{1}\right)$. Plus généralement on peut définir pour $n \geq 1$ l'application $R_{n}: D_{n} \mapsto\left(X_{n}, Z_{n}\right)$. On note $\Gamma_{n}(r)$ l'image de $D_{n}$ par $R_{n}$ en utilisant des géodésiques de longueur $r$. L'ensemble $\Gamma_{n}(r)$ est l'union des deux ensembles notés $\Gamma_{n}^{\sigma}(r), \sigma= \pm 1$ correspondant respectivement à $\varphi \in\left[-\frac{\pi}{2},+\frac{\pi}{2}\right]$ et $\left.\varphi \in\right] \frac{\pi}{2}, \frac{3 \pi}{2}[$. Si $a: t \mapsto( \pm t, 0,0)$ est la géodésique anormale paramétrisée par la longueur d'arc, la trace $W(0, r) \cap\{y=0\}$ est l'union de l'ensemble $W_{1}=( \pm r, 0) \cup_{n \geq 1} \Gamma_{n}(r)$ et de son symétrique $-W_{1}$.

\subsubsection{Lemmes préliminaires}

De notre étude préliminaire de la section 4.5 on déduit les lemmes suivants (N.B. la contrainte $|y \varepsilon|<1$ n'est pas ici prise en compte).

Lemme 4.13. Le domaine de définition $D_{1}$ est l'ensemble

$$
(\lambda=0, \varphi \neq n \pi) \cup\left(\lambda>0, k^{\prime \prime} \neq 0\right) \cup\left(\lambda>0, k^{\prime \prime}=0, \varphi \in\right]-\frac{\pi}{2}, 0[\cup] \frac{\pi}{2}, \pi[) .
$$

Lemme 4.14. Si $n \geq 2$, le domaine $D_{n}$ est l'ensemble

$$
(\lambda=0, \varphi \neq n \pi) \cup\left(\lambda>0, k^{\prime \prime} \neq 0\right) .
$$

\section{Lemme 4.15.}

1. Si $p_{x}=1$ et $k^{\prime \prime} \neq 0$, alors $t_{n}$ est $n \mathcal{P}$ où $\mathcal{P}$ est la période d'oscillation de $y$ définie par (4.13).

2. Si $p_{x}=-1$ alors $k^{\prime \prime}<0$ et $t_{n}=n \mathcal{P}$.

3. Si $p_{x} \neq \pm 1, t_{2 n}(\varphi, \lambda)=t_{2 n}(\pi-\varphi, \lambda)=n \mathcal{P}$ et $\left(X_{2 n}(\varphi, \lambda), Z_{2 n}(\varphi, \lambda)\right)=\left(X_{2 n}(\pi-\varphi, \lambda), Z_{2 n}(\pi-\varphi, \lambda)\right)$.

Preuve. Il faut observer que si $p_{x}^{2}=1$ la coordonnée $y$ oscille dans les cas A,B et D périodiquement entre $y_{-}$et $y_{+}$où $y_{-}$est ici 0 . On a alors toujours $\sigma=+1$ et la trajectoire est entièrement contenue dans $y \geq 0$. Si $p_{x}^{2} \neq 1$, la coordonnée $y$ oscille dans les cas A,B,D $(\varphi \neq n \pi)$ entre $y_{-}$et $y_{+}$où $y_{-}<0<y_{+}$. Pour chaque $p_{x}=\sin \varphi$, $\varphi \in]-\frac{\pi}{2},+\frac{\pi}{2}$ [ il existe un mouvement correspondant à $(\varphi, \lambda)$ où $\sigma=+1$ et un mouvement correspondant à $(\pi-\varphi, \lambda)$ où $\sigma=-1$. Pour ces deux mouvements, $y_{-}$et $y_{+}$sont identiques et la période est la même. Les intersections paires des géodésiques correspondantes avec $y=0$ sont les mêmes et ont même période (voir la proposition 2.6, valide dans le cas intégrable général).

On en déduit le corollaire important suivant, voir la figure 19.

Corollaire 4.16. Dans les cas $A$ et $B, p_{x}^{2} \neq 1$, une géodésique de longueur $L(e)>\mathcal{P}$ n'est pas minimisante.

Remarque 4.17. Dans le cas plat, une géodésique où $y$ est périodique cesse d'être minimisante à sa première intersection avec le plan $y=0 \mathrm{car}$ du fait de la symétrie, à ce point la géodésique associée à $\varphi$ rencontre celle associée à $\pi-\varphi$. Dans le cas non plat, du fait de la propriété d'intégrabilité les secondes intersections avec $y=0$ coincident. Cette propriété est importante car pour le calcul de la sphère on peut supposer $L(e) \leq \mathcal{P}$ dans les cas $\mathrm{A}, \mathrm{B}$ avec $p_{x}^{2} \neq 1$.

Une remarque géométrique importante est la suivante.

Lemme 4.18. Supposons $k^{\prime \prime}<0, p_{x}<0$ et $\sigma=-1$. Alors on a: $X_{1}(\varphi, \lambda)<0$ et $Z_{1}(\varphi, \lambda)<0$ dans $U$.

Preuve. Cela résulte de l'analyse de la section 4.5 sur le graphe de $P_{1}$. Dans le cas $\mathrm{B}$, si $p_{x}<0$ alors les deux racines $y_{3}, y_{4}$ de $P_{1}=-1$ sont négatives et $y$ oscille entre $y_{4}$ et $y_{2}$. Si $\sigma=-1$, alors $y$ se déplace d'abord entre 0 et $y_{4}$ et $P_{1}(y)<0$ dans cet intervalle car $P_{1}(0)=p_{x}$ et $P_{1}\left(y_{4}\right)=-1$. Les formules intégrales de la section 4.6 montrent que $X_{1}$ et $Z_{1}$ sont $<0$. 


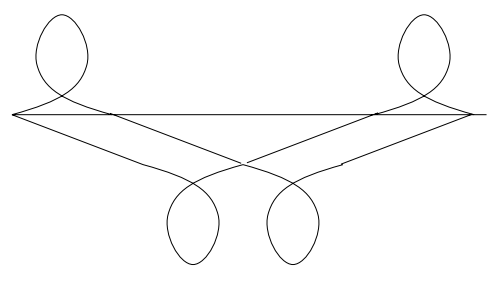

Cas A

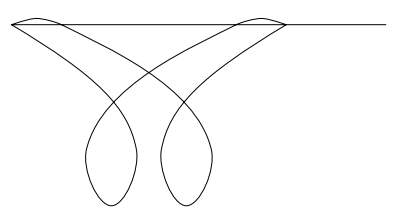

Cas B

Figure 19

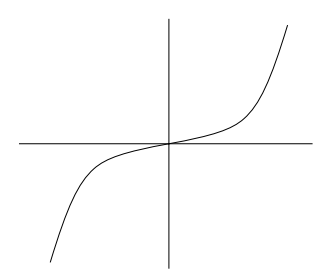

(i) $\wedge_{0}$

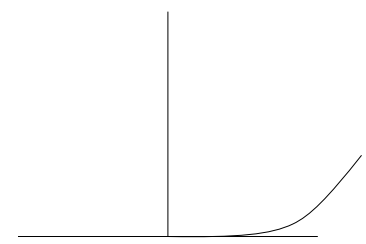

(iii) $B_{1}$

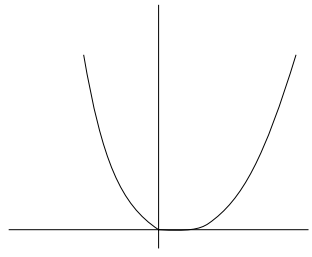

(ii) $K_{0}^{\prime \prime}$

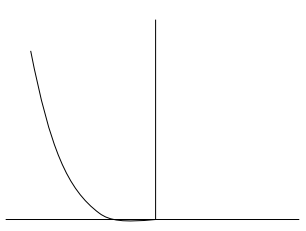

(iv) $B_{-1}$

FigURE 20

\subsubsection{Notations}

À partir de maintenant on localise notre étude en 0 et l'on ne va considérer que des géodésiques dont la longueur est $\leq \eta$ où $\eta$ est choisi assez petit. Elles recouvrent un voisinage de 0 noté $U_{\eta}$. Une première étape dans notre analyse est de représenter les ensembles $K_{0}^{\prime \prime}, \Lambda_{0}, B_{1}$ et $B_{-1}$ qui sont les images respectives par l'application $R_{1}$ des ensembles $k^{\prime \prime}=0, \lambda=0, p_{x}=1$ et $p_{x}=-1$, en se limitant à des courbes de longueur $\leq \eta$. Ce sont des ensembles que l'on peut paramétriser aisément et qui vont former des courbes ramifiées en 0 .

4.12.5. Représentation de $K_{0}^{\prime \prime}, \Lambda_{0}, B_{1}, B_{-1}$

On représente sur la figure 20, ces courbes ramifiées en 0 .

\subsubsection{Description de $\Gamma_{1}^{+}(r)$}

Cet ensemble correspond à $\varphi \in\left[-\frac{\pi}{2}, \frac{\pi}{2}\right]$ et le calcul montre que $\Gamma_{1}^{+}(r)$ est l'image par l'application $R_{1}$ d'une courbe $C^{0}$ notée $\gamma_{1}$ dans l'espace des paramètres. On la représente sur la figure 21 de même que son image $\Gamma_{1}^{+}(r)$ en figure 22, l'orientation indiquant la correspondance entre les points.

Une propriété géométrique importante est que contrairement au cas plat, la courbe $\Gamma_{1}^{+}(r)$ n'a pas ses deux extrémités en $(-r, 0)$ et $(r, 0)$ sur la direction anormale, mais sur les courbes $B_{1}$ et $B_{-1}$. La partie hachurée située au-dessus de $\gamma_{1}$ est envoyée sur le domaine hachuré limité par $\Gamma_{1}^{+}(r), B_{-1}$ et $B_{1}$. 


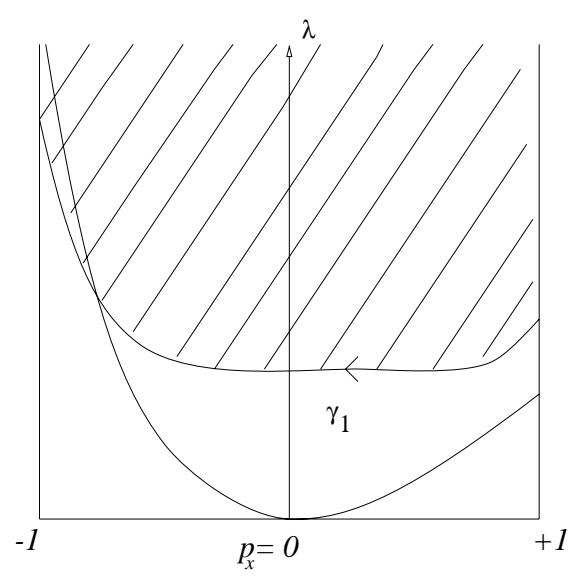

FIGURE 21

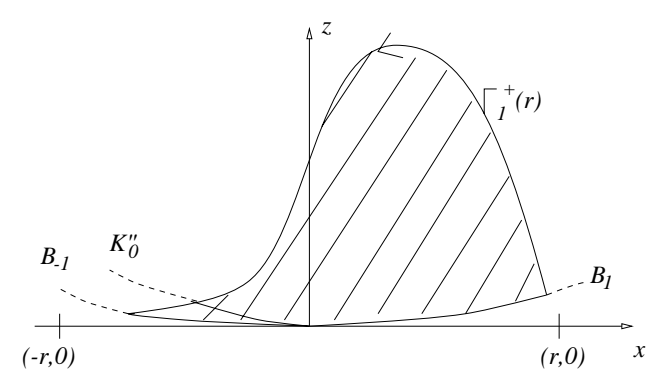

FIGURE 22

\subsubsection{Description de $\Gamma_{1}^{-}(r)$}

Cette courbe est obtenue pour $\varphi \in] \frac{\pi}{2}, \frac{3 \pi}{2}$ [ et est l'image d'une courbe formée de deux composantes connexes $\gamma_{2}$ et $\gamma_{3}$ dans l'espace des paramètres, voir figures 23,24 . On note $c_{2}$ et $c_{3}$ ces images respectives.

La propriété géométrique essentielle est l'existence de l'axe $p_{x}=-1$ comme direction asymptotique pour $\gamma_{2}$ et $\gamma_{3}$. Cette propriété s'explique aisément : les géodésiques associées à $\left.k^{\prime \prime}=0, \varphi \in\right] \frac{\pi}{2}, \frac{3 \pi}{2}$ [ ne réintersectent pas le plan $y=0$ et l'ensemble $k^{\prime \prime}=0$ est donc une direction asymptotique pour $\gamma_{2}$ et $\gamma_{3}$. Les images respectives de cette direction singulière sont le point $(-r, 0)$ extrémité de la géodésique anormale. Les deux autres extrémités de $c_{2}, c_{3}$ sont formées respectivement des points $Q$ et $-Q$ et correspondent à $\lambda=0$. Observons aussi que la courbe $c_{2}$ est entièrement située dans le domaine $z<0$ et c'est une conséquence du lemme 4.18. Les parties hachurées dans l'espace des paramètres s'appliquent dans le domaine hachuré limité par $c_{2}, c_{3}$ et $\Lambda_{0}$.

Le cas plat s'obtient topologiquement par passage à la limite en écrasant $\Lambda_{0}$ et $K_{0}^{\prime \prime}$ sur l'axe $O x$ correspondant à la direction anormale, voir figure 25 .

4.12.8. Représentation de la courbe $\Gamma_{2}^{+}(r)=\Gamma_{2}^{-}(r)$

Cette courbe est l'image de l'application $R_{2}$. Par symétrie on peut supposer $\varphi \in\left[-\frac{\pi}{2},+\frac{\pi}{2}\right]$. Observons tout d'abord que l'on peut supposer $k^{\prime \prime} \neq 0$ et $\lambda \neq 0$. En effet les géodésiques associées à $k^{\prime \prime}=0$ ont au plus une intersection avec $y=0$ et par ailleurs celles associées à $\lambda=0$ sont de grande longueur quand elles réintersectent pour la seconde fois le plan $y=0$. 


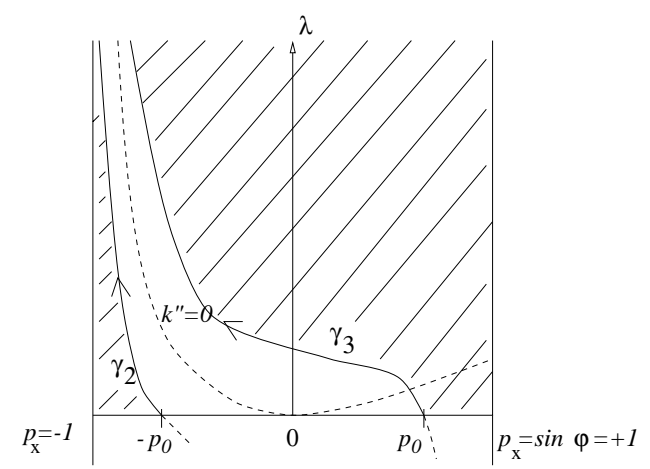

FIGURE 23

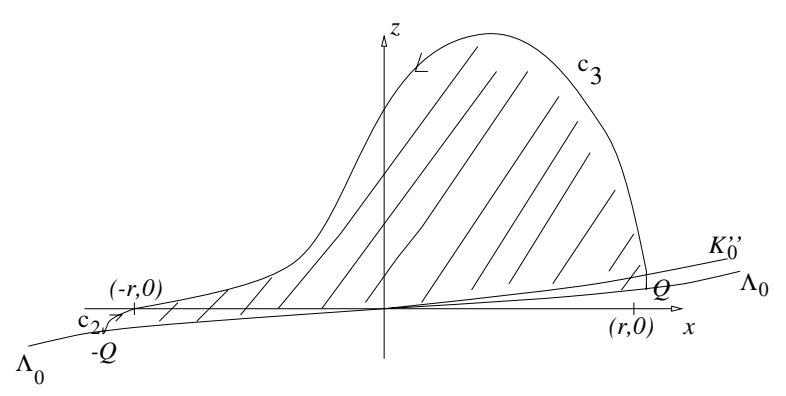

FiguRe 24

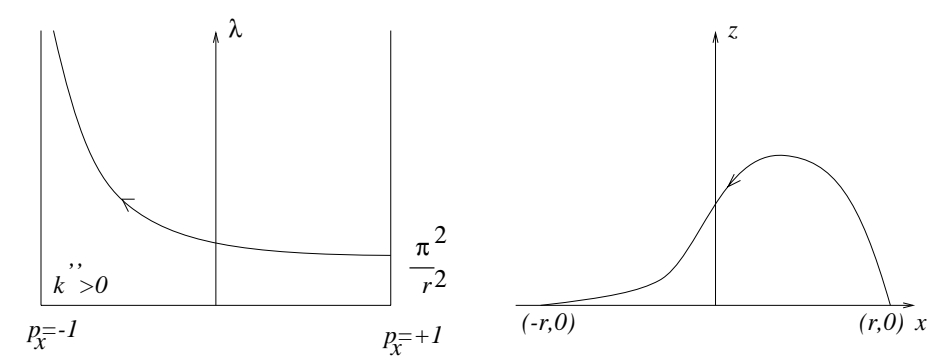

FIGURE 25

Notons $B_{2}$ et $B_{-2}$ les images respectives de $\varphi=+\frac{\pi}{2}$ et $\varphi=-\frac{\pi}{2}$ par $R_{2}$. Un calcul facile montre que les courbes $B_{1}, B_{2}$ et $B_{-1}, B_{-2}$ sont homothétiques et l'on a

$$
B_{2}=2 B_{1}, \quad B_{-2}=2 B_{-1}
$$

ce qui permet de positionner ces courbes. 


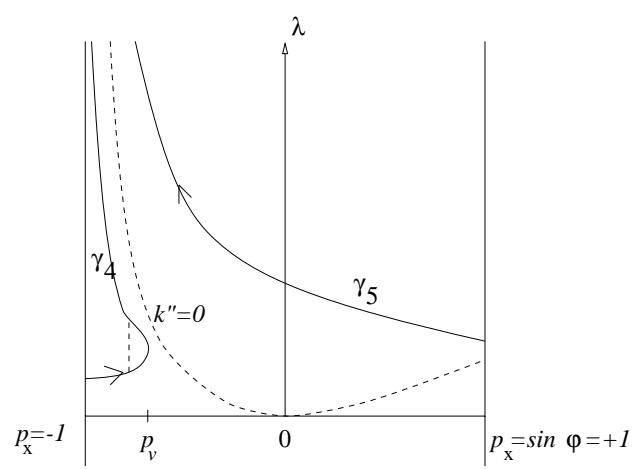

FIGURE 26
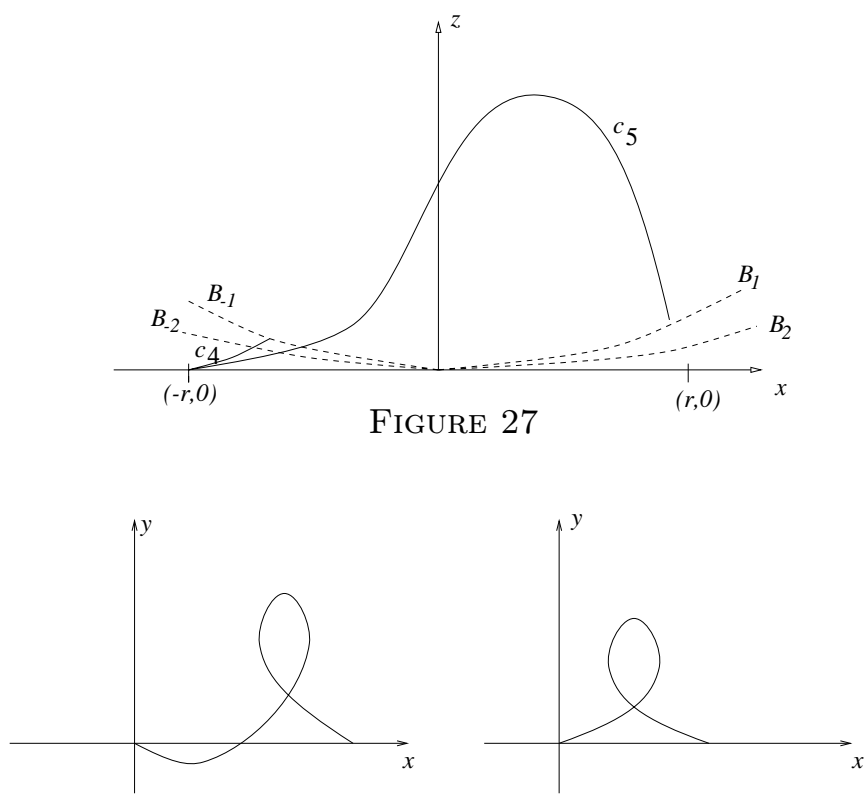

Cas $B: p \rightarrow-1$

Cas limite : $p_{x}^{2}=1$

Figure 28

Le calcul des secondes intersections des géodésiques avec le plan $y=0$ dans les cas A et B est simple car la seconde intersection a lieu exactement après une période $\mathcal{P}$ soit donc les relations :

Cas A : $r \sqrt{\lambda}=4 K(k)$.

Cas B : $r k \sqrt{\lambda}=2 K(\hat{k})$.

On représente sur la figure 27 , la courbe $\Gamma_{2}^{+}(r)$. C'est l'image d'une courbe formée de deux composantes connexes $\gamma_{4}$ et $\gamma_{5}$ dans l'espace des paramètres et on note $c_{4}$ et $c_{5}$ les images correspondantes. Les propriétés géométriques essentielles sont les suivantes. Les deux courbes $\gamma_{4}$ et $\gamma_{5}$ ont l'axe $p_{x}=-1$ comme direction asymptotique et les images se ramifient en $(-r, 0)$. La courbe $\gamma_{4}$ possède ici une tangente verticale. Une extrémité de $\gamma_{4}$ et de $\gamma_{5}$ est située respectivement sur $p_{x}=-1$ et $p_{x}=1$ et les images se raccordent avec $\Gamma_{1}^{ \pm}$ en les points $b_{-1}$ et $b_{1}$, voir l'explication géométrique de ce phénomène sur la figure 28 .

Enfin, les images de $p_{x}^{2}=+1$ sont deux points isolés $b_{-2}$ et $b_{2}$ sur les courbes $B_{-2}$ et $B_{2}$. 


\subsection{Développement asymptotiques}

L'objectif de cette section est d'évaluer la transcendance précise nécessaire pour paramétriser dans les coordonnées $(x, z)$ les courbes $c_{2}, c_{3}, c_{4}$ et $c_{5}$ au voisinage du point $(-r, 0)$. Ces courbes sont les images respectives par les applications $R_{1}$ ou $R_{2}$ des courbes $\gamma_{2}, \gamma_{3}, \gamma_{4}$ et $\gamma_{5}$.

Pour calculer le développement asymptotique $c_{3}$ on utilise les formules intégrales de la section 4.6 et l'on a :

$$
\begin{aligned}
& X_{1}=2 \int_{0}^{y_{\sigma}} \frac{\sigma(1+\varepsilon y) P_{1}(y) d y}{\sqrt{1-P_{1}^{2}(y)}}=\frac{2}{\sqrt{\lambda}} \int_{-1}^{\alpha} F(\eta, \varepsilon, \varphi, \lambda) d \eta \\
& Z_{1}=\int_{0}^{y_{\sigma}} \frac{\sigma y^{2} P_{1}(y) d y}{\sqrt{1-P_{1}^{2}(y)}}=\frac{1}{\lambda^{\frac{3}{2}}} \int_{-1}^{\alpha} G(\eta, \varepsilon, \varphi, \lambda) d \eta
\end{aligned}
$$

où l'on pose

$$
\begin{aligned}
\alpha & =\frac{\varepsilon p_{x}}{2 k \sqrt{\lambda}} \\
F & =\frac{\left(1+2 k \eta \frac{\varepsilon}{\sqrt{\lambda}}-\frac{\varepsilon^{2}}{\lambda} p_{x}\right)\left(2 k^{2} \eta^{2}+p_{x}-\frac{\varepsilon^{2} p_{x}^{2}}{2 \lambda}\right)}{D} \\
G & =\frac{\left(2 k \eta-\frac{\varepsilon p_{x}}{\sqrt{\lambda}}\right)^{2}\left(2 k^{2} \eta^{2}+p_{x}-\frac{\varepsilon^{2} p_{x}^{2}}{2 \lambda}\right)}{D} \\
D & =\sqrt{\left(1-\eta^{2}\right)\left(k^{\prime \prime}+k^{2} \eta^{2}\right)}
\end{aligned}
$$

et la longueur fixée à $r$ est donnée par :

$$
\theta=r=\frac{2}{\sqrt{\lambda}} \int_{-1}^{\alpha} \frac{d \eta}{D}
$$

On remarque que $F$ et $G$ sont des fonctions polynômiales en $\frac{\varepsilon}{\sqrt{\lambda}}$ et que les intégrales à évaluer sont toutes de la forme :

$$
\int \frac{P(\eta) d \eta}{\sqrt{\left(1-\eta^{2}\right)\left(k^{\prime 2}+k^{2} \eta^{2}\right)}}
$$

où $P(\eta)$ est un polynôme en $\eta$. On cherche des développements lorsque $p_{x} \rightarrow-1$ et $\lambda \rightarrow+\infty$, on a alors $k^{\prime} \rightarrow 0$ et $k \rightarrow 1$ avec :

$$
\begin{gathered}
2 k^{2}=1-p_{x}+\frac{\varepsilon^{2} p_{x}^{2}}{2 \lambda} \\
2 k^{\prime 2}=1+p_{x}-\frac{\varepsilon^{2} p_{x}^{2}}{2 \lambda} .
\end{gathered}
$$

On observe que l'on peut choisir $\sqrt{\lambda}$ et $k^{\prime 2}$ comme variables, le paramètre $p_{x}$ étant calculé en résolvant l'équation du second ordre :

$$
-\frac{\varepsilon^{2} p_{x}^{2}}{2 \lambda}+p_{x}+\left(1-2 k^{\prime 2}\right)=0
$$


et en conservant la branche :

$$
p_{x}=\frac{\lambda}{\varepsilon^{2}}\left(1-\sqrt{1+\frac{2 \varepsilon^{2}}{\lambda}\left(1-2 k^{\prime 2}\right)}\right)
$$

qui est au voisinage de 0 une fonction analytique de la forme

$$
p_{x}=A_{1}\left(\frac{\varepsilon^{2}}{\lambda}, k^{\prime 2}\right), p_{x} \sim-1 .
$$

Le phénomène important est le suivant. En fixant la longueur des géodésiques à $r$ il vient :

$$
\frac{r \sqrt{\lambda}}{2}=\int_{-1}^{\alpha} \frac{d \eta}{\sqrt{\left(1-\eta^{2}\right)\left(k^{\prime 2}+k^{2} \eta^{2}\right)}}
$$

où $\alpha>0$. On peut donc écrire :

$$
\frac{r \sqrt{\lambda}}{2}=\int_{-1}^{0} \frac{d \eta}{\sqrt{\left(1-\eta^{2}\right)\left(k^{\prime 2}+k^{2} \eta^{2}\right)}}+\int_{0}^{\alpha} \frac{d \eta}{\sqrt{\left(1-\eta^{2}\right)\left(k^{\prime 2}+k^{2} \eta^{2}\right)}}
$$

La première intégrale est l'intégrale complète $K\left(k^{\prime}\right)$ qui admet lorsque $k^{\prime} \rightarrow 0$ l'écriture :

$$
K\left(k^{\prime}\right)=u_{3}\left(k^{\prime 2}\right) \ln \frac{4}{k^{\prime}}+u_{4}\left(k^{\prime 2}\right)
$$

où $u_{3}, u_{4}$ sont des fonctions analytiques en 0 et

$$
u_{3}\left(k^{\prime 2}\right)=1+o(1), \quad u_{4}\left(k^{\prime 2}\right)=o(1) .
$$

En particulier cela implique que

$$
K\left(k^{\prime}\right)=\ln \frac{4}{k^{\prime}}+o\left(k^{\prime}\right)
$$

et donc :

$$
\frac{1}{K\left(k^{\prime}\right)}=\frac{1}{\ln \frac{4}{k^{\prime}}}\left(1+A_{2}\left(k^{\prime 2}, \frac{1}{\ln \frac{4}{k^{\prime}}}\right)\right)
$$

où $A_{2}$ est analytique en 0 et $A(0)=0$.

La seconde intégrale est majorée par $K\left(k^{\prime}\right)$ et pour l'étudier on observe que lorsque $\lambda \rightarrow+\infty, \alpha=o(1)$. En développant $\frac{1}{\sqrt{1-\eta^{2}}}$ au voisinage de 0 , on obtient le développement suivant :

$$
\int_{0}^{\alpha} \frac{d \eta}{\sqrt{\left(1-\eta^{2}\right)\left(k^{\prime 2}+k^{2} \eta^{2}\right)}}=\int_{0}^{\alpha} \frac{d \eta}{\sqrt{k^{\prime 2}+k^{2} \eta^{2}}}+\sum_{n \geq 1} B_{n} \int_{0}^{\alpha} \frac{\eta^{2 n} d \eta}{\sqrt{k^{\prime 2}+k^{2} \eta^{2}}} .
$$

Pour $n \geq 0$, les intégrales de la forme :

$$
\int_{0}^{\alpha} \frac{\eta^{2 n} d \eta}{\sqrt{k^{\prime 2}+k^{2} \eta^{2}}}=\int_{0}^{\alpha} \frac{\eta^{2 n} d \eta}{k^{\prime} \sqrt{1+\left(\frac{k \eta}{k^{\prime}}\right)^{2}}}
$$


s'évaluent aisément en posant :

$$
v=\frac{k \eta}{k^{\prime}}, s=\sinh t
$$

et il vient :

$$
\int_{0}^{\alpha} \frac{\eta^{2 n} d \eta}{\sqrt{k^{\prime 2}+k^{2} \eta^{2}}}=\int_{0}^{\operatorname{Arcsinh} \frac{k}{k^{\prime}} \alpha} \frac{k^{\prime 2 n}}{k^{2 n+1}} \sinh ^{2 n} t d t
$$

où la primitive $I_{n}=\int \sinh ^{2 n} t d t=\int P\left(\mathrm{e}^{t}, \mathrm{e}^{-t}\right) d t$ peut se calculer par récurrence. En particulier on en déduit la relation suivante :

$$
\frac{r \sqrt{\lambda}}{2}=K\left(k^{\prime}\right)+\operatorname{Arcsinh} \frac{k \alpha}{k^{\prime}}+A_{3}\left(\frac{\varepsilon^{2}}{\lambda}\right)+o\left(k^{\prime}\right)
$$

où $A_{3}$ est une fonction analytique en 0 et $A_{3}(0)=0$.

L'équation précédente implique donc lorsque $\lambda \rightarrow+\infty$ les estimées :

$$
\frac{r \sqrt{\lambda}}{2} \sim 2 \ln \frac{1}{k^{\prime}}, \quad k^{\prime}=o\left(\frac{1}{\sqrt{\lambda}}\right)
$$

et en particulier $\frac{k \alpha}{k^{\prime}} \rightarrow+\infty$. On peut donc écrire avec $\alpha=\frac{\varepsilon p_{x}}{2 k \sqrt{\lambda}}$

$$
\frac{r \sqrt{\lambda}}{2}=2 \ln \frac{1}{k^{\prime}}-\ln \sqrt{\lambda}+\ln 4+\ln -\frac{\varepsilon}{2}+o\left(\frac{1}{\sqrt{\lambda}}\right) .
$$

En écrivant :

$$
\frac{r \sqrt{\lambda}}{2}=2 \ln \frac{1}{k^{\prime}}\left(1+y_{1}\right), \quad y_{1} \rightarrow 0
$$

et en résolvant l'équation à l'aide du théorème des fonctions implicites il vient :

$$
y_{1}=\frac{1}{2 \ln \frac{1}{k^{\prime}}}\left[-\ln _{2} \frac{1}{k^{\prime}}+\ln \left(-\frac{\varepsilon r}{2}\right)+o(1)\right]
$$

où $\ln _{2}$ désigne la fonction composée de deux logarithmes. Soit donc la relation fondamentale

$$
\frac{r \sqrt{\lambda}}{2}=2 \ln \frac{1}{k^{\prime}}-\ln _{2} \frac{1}{k^{\prime}}+\ln \left(-\frac{\varepsilon r}{2}\right)+o(1) .
$$

Le cas plat $\varepsilon=0$ ne s'obtient pas par passage à la limite $\varepsilon \rightarrow 0$ et la formule du cas plat est :

$$
\frac{r \sqrt{\lambda}}{2}=K\left(k^{\prime}\right)=\ln \frac{1}{k^{\prime}}+\ln 4+o(1) .
$$

En particulier à $k^{\prime}$ fixé le second membre est d'ordre $2 \ln \frac{1}{k^{\prime}}$ pour $\varepsilon \neq 0$ contre $\ln \frac{1}{k^{\prime}}$ dans le cas plat, soit le double. Ce qui s'explique aisément car lorsque $k^{\prime} \rightarrow 0$, la valeur de l'intégrale $\int_{0}^{1} \frac{d \eta}{\sqrt{k^{\prime 2}+k^{2} \eta^{2}}}$ est concentrée au voisinage de 0 . 
Par ailleurs en utilisant la formule :

$$
\ln k^{\prime}=-\frac{r \sqrt{\lambda}}{4}+\frac{1}{2} \ln \left(-\frac{\varepsilon}{2 \sqrt{\lambda}}\right)+\ln 2+o(1)
$$

il vient

$$
k^{\prime}=2 \sqrt{-\frac{\varepsilon}{2 \sqrt{\lambda}}} \mathrm{e}^{-\frac{r \sqrt{\lambda}}{4}}(1+o(1))
$$

Proposition 4.19. On a les estimées suivants lorsque $k^{\prime}, \frac{1}{\sqrt{\lambda}} \rightarrow 0$ tout en satisfaisant la contrainte $\theta=r$ :

- $\frac{r \sqrt{\lambda}}{2}=2 \ln \frac{1}{k^{\prime}}-\ln _{2} \frac{1}{k^{\prime}}+\ln \left(-\frac{\varepsilon r}{2}\right)+o(1)$

- $k^{\prime}=2 \sqrt{-\frac{\varepsilon}{2 \sqrt{\lambda}}} \mathrm{e}^{-\frac{r \sqrt{\lambda}}{4}}(1+o(1))$

- $\frac{2}{r \sqrt{\lambda}}=\frac{1}{2 \ln \frac{1}{k^{\prime}}}\left(1+\frac{\ln _{2} \frac{1}{k^{\prime}}}{2 \ln \frac{1}{k^{\prime}}}+o\left(\frac{\ln _{2} \frac{1}{k^{\prime}}}{\ln \frac{1}{k^{\prime}}}\right)\right)$.

En particulier dans le cas $\varepsilon \neq 0$ il est donc nécessaire d'utiliser des composés de deux logarithmes pour évaluer la sphère.

Par ailleurs :

$$
X_{1}=\frac{2}{\sqrt{\lambda}} \int_{-1}^{\alpha} F(\eta, \varepsilon, \varphi, \lambda) d \eta
$$

$\operatorname{avec} F=\frac{N}{D}$ et $=$

$$
\begin{aligned}
& N=\left(1+2 k \eta \frac{\varepsilon}{\sqrt{\lambda}}-\frac{\varepsilon^{2}}{\lambda} p_{x}\right)\left(2 k^{2} \eta^{2}+p_{x}-\frac{\varepsilon^{2} p_{x}}{2 \lambda}\right) \\
& D=\sqrt{\left(1-\eta^{2}\right)\left(k^{\prime 2}+k^{2} \eta^{2}\right)} .
\end{aligned}
$$

On écrit :

$$
\begin{aligned}
N & =\left(2 k^{2} \eta^{2}+\left(2 k^{\prime 2}-1\right)\right)\left(1+2 k \eta \frac{\varepsilon}{\sqrt{\lambda}}-\frac{\varepsilon^{2}}{\lambda} p_{x}\right) \\
& =\left(2\left(k^{2} \eta^{2}+k^{\prime 2}\right)-1\right)+\frac{\varepsilon k \eta}{\sqrt{\lambda}}\left(2\left(k^{2} \eta^{2}+k^{\prime 2}\right)-1\right)-\frac{\varepsilon^{2}}{\lambda} p_{x}\left(2\left(k^{2} \eta^{2}+k^{\prime 2}\right)-1\right) .
\end{aligned}
$$

Soit donc :

$$
X_{1}=\frac{2}{\sqrt{\lambda}}\left[\int_{-1}^{\alpha} \frac{2\left(k^{2} \eta^{2}+k^{\prime 2}\right)-1}{D} d \eta+\frac{\varepsilon}{\sqrt{\lambda}} \int_{-1}^{\alpha} \frac{2 k \eta\left(2\left(k^{2} \eta^{2}+k^{\prime 2}\right)-1\right)}{D} d \eta-\frac{\varepsilon^{2}}{\lambda} p_{x} \int_{-1}^{\alpha} \frac{\left(2\left(k^{2} \eta^{2}+k^{\prime 2}\right)-1\right)}{D} d \eta\right]
$$

et l'on obtient donc :

$$
X_{1}=I_{0}+\frac{2}{\sqrt{\lambda}} I_{1}+\frac{2}{\sqrt{\lambda}} I_{2}+\frac{2}{\sqrt{\lambda}} I_{3}
$$


avec

$$
\begin{aligned}
& I_{0}=-\frac{2}{\sqrt{\lambda}} \int_{-1}^{\alpha} \frac{d \eta}{D}=-\theta=-r \\
& I_{1}=\int_{-1}^{\alpha} \frac{2 \sqrt{k^{2} \eta^{2}+k^{\prime 2}} d \eta}{\sqrt{1-\eta^{2}}} \\
& I_{2}=\frac{\varepsilon}{\sqrt{\lambda}}\left[\int_{-1}^{\alpha} \frac{4 k \eta \sqrt{k^{2} \eta^{2}+k^{\prime 2}} d \eta}{\sqrt{1-\eta^{2}}}-\int_{-1}^{\alpha} \frac{2 k \eta d \eta}{D}\right] \\
& I_{3}=-\frac{\varepsilon^{2}}{\lambda} p_{x} \int_{-1}^{\alpha} \frac{2 \sqrt{k^{2} \eta^{2}+k^{\prime 2}} d \eta}{\sqrt{1-\eta^{2}}}+\frac{\varepsilon^{2}}{2 \sqrt{\lambda}} p_{x} r .
\end{aligned}
$$

Toutes ces intégrales s'évaluent aisément en utilisant la transformation déjà décrite précédement :

$$
v=\frac{k \eta}{k^{\prime}}, v=\sinh t
$$

Par ailleurs observons le fait suivant. On a $\alpha=\frac{\varepsilon p_{x}}{2 k \sqrt{\lambda}}$, donc pour $\varepsilon=0, \alpha=0$ et il vient :

$$
I_{1 \mid \varepsilon=0}=\int_{-1}^{0} \frac{2 \sqrt{k^{2} \eta^{2}+k^{\prime 2}} d \eta}{\sqrt{1-\eta^{2}}}=2 E(k)
$$

et $I_{2 \mid \varepsilon=0}=I_{3 \mid \varepsilon=0}=0$. Donc pour $\varepsilon=0$, on a bien le résultat obtenu dans le cas plat :

$$
X_{1}+r=\frac{4 E(k)}{\sqrt{\lambda}}
$$

Évaluons $I_{1}$. On écrit :

$$
I_{1}=\int_{-1}^{0} \frac{2 \sqrt{k^{2} \eta^{2}+k^{\prime 2}} d \eta}{\sqrt{1-\eta^{2}}}+\int_{0}^{\alpha} \frac{2 \sqrt{k^{2} \eta^{2}+k^{\prime 2}} d \eta}{\sqrt{1-\eta^{2}}}=2 E(k)+I_{1}^{\prime},
$$

où l'on a posé :

$$
I_{1}^{\prime}=\int_{0}^{\alpha} \frac{2 \sqrt{k^{2} \eta^{2}+k^{\prime 2}} d \eta}{\sqrt{1-\eta^{2}}} .
$$

On a : $\alpha=o(1)$ et $\alpha$ est une fonction analytique en $\frac{1}{\sqrt{\lambda}}$ et $k^{\prime 2}$. On peut écrire :

$$
I_{1}^{\prime}=2 \int_{0}^{\alpha} \frac{\eta d \eta}{\sqrt{1-\eta^{2}}}+o\left(k^{\prime}\right)=\alpha^{2}+o\left(\alpha^{2}\right)+o\left(k^{\prime}\right) .
$$

On obtient donc :

$$
I_{1}=\left(2+\alpha^{2}\right)+o\left(\alpha^{2}\right)+o\left(k^{\prime}\right)
$$

où le terme en $o\left(k^{\prime}\right)$ est en fait de la forme $O\left(k^{\prime 2} \ln \frac{1}{k^{\prime}}\right)$.

Évaluons maintenant $I_{2}$. On écrit :

$$
I_{2}=\frac{\varepsilon}{\sqrt{\lambda}}\left(I_{2}^{\prime}+I_{2}^{\prime \prime}\right)
$$


où l'on a posé

$$
I_{2}^{\prime}=\int_{-1}^{\alpha} \frac{4 k \eta \sqrt{k^{2} \eta^{2}+k^{\prime 2}} d \eta}{\sqrt{1-\eta^{2}}}, \quad I_{2}^{\prime \prime}=-\int_{-1}^{\alpha} \frac{2 k \eta d \eta}{D}
$$

On a

$$
I_{2}^{\prime}=\int_{-1}^{\alpha} \frac{4 \eta|\eta| d \eta}{\sqrt{1-\eta^{2}}}+o\left(k^{\prime}\right)
$$

et donc

$$
I_{2}^{\prime}=-\int_{-1}^{0} \frac{4 \eta^{2} d \eta}{\sqrt{1-\eta^{2}}}+o\left(\alpha^{2}\right)+o\left(k^{\prime}\right)
$$

où le terme intégral se calcule aisément en posant $\eta=\sin \theta$. On obtient :

$$
I_{2}^{\prime}=-\pi+o\left(\alpha^{2}\right)+o\left(k^{\prime}\right) .
$$

De même :

$$
\begin{aligned}
I_{2}^{\prime \prime} & =-\int_{-1}^{\alpha} \frac{2 k \eta d \eta}{\sqrt{\left(1-\eta^{2}\right)\left(k^{\prime 2}+k^{2} \eta^{2}\right)}}=-\int_{-1}^{\alpha} \frac{2 \eta d \eta}{|\eta| \sqrt{1-\eta^{2}}}+o\left(k^{\prime}\right) \\
& =2 \int_{-1}^{0} \frac{d \eta}{\sqrt{1-\eta^{2}}}-2 \int_{0}^{\alpha} \frac{d \eta}{\sqrt{1-\eta^{2}}}+o\left(k^{\prime}\right)=\pi-2 \alpha+o\left(\alpha^{2}\right)+o\left(k^{\prime}\right) .
\end{aligned}
$$

D'où l'estimée :

$$
I_{2}=\frac{\varepsilon}{\sqrt{\lambda}}\left(-2 \alpha+o\left(\alpha^{2}\right)+o\left(k^{\prime}\right)\right)
$$

Enfin on a :

$$
I_{3}=-\frac{\varepsilon^{2}}{\lambda} p_{x} I_{1}+\frac{\varepsilon^{2}}{2 \sqrt{\lambda}} p_{x} r
$$

On obtient donc

$$
\frac{X_{1}+r}{2}=\frac{1}{\sqrt{\lambda}}\left(I_{1}+I_{2}+I_{3}\right)=\frac{1}{\sqrt{\lambda}}\left[2-\frac{\varepsilon^{2} r}{2 \sqrt{\lambda}}+o\left(\frac{1}{\sqrt{\lambda}}\right)\right]+o\left(k^{\prime}\right) .
$$

En particulier on peut utiliser cette formule pour calculer les deux premiers termes du développement de $\frac{1}{\sqrt{\lambda}}$ comme fonction analytique en $X=\frac{X_{1}+r}{2}$, aux termes en $k^{\prime}$ près. Il vient :

$$
\frac{1}{\sqrt{\lambda}}=\frac{X}{2}+\frac{\varepsilon^{2} r}{16} X^{2}+o\left(X^{2}\right)
$$

Calculons maintenant $Z_{1}$. On a :

$$
\begin{aligned}
\lambda^{\frac{3}{2}} Z_{1} & =\int_{-1}^{\alpha} \frac{\left(2 k \eta-\frac{\varepsilon p_{x}}{\sqrt{\lambda}}\right)^{2}\left(2 k^{2} \eta^{2}+2 k^{\prime 2}-1\right) d \eta}{\sqrt{\left(1-\eta^{2}\right)\left(k^{\prime 2}+k^{2} \eta^{2}\right)}} \\
& =\int_{-1}^{\alpha} \frac{\left(4 k^{2} \eta^{2}-\frac{4 k \eta \varepsilon p_{x}}{\sqrt{\lambda}}+\frac{\varepsilon^{2} p_{x}^{2}}{\lambda}\right)\left(2\left(k^{2} \eta^{2}+k^{\prime} 2\right)-1\right) d \eta}{\sqrt{\left(1-\eta^{2}\right)\left(k^{\prime 2}+k^{2} \eta^{2}\right)}}=J_{1}+J_{2}+J_{3}
\end{aligned}
$$


où l'on a posé :

$$
\begin{aligned}
& J_{1}=\int_{-1}^{\alpha} \frac{8 k^{2} \eta^{2} \sqrt{k^{2} \eta^{2}+k^{\prime 2}} d \eta}{\sqrt{1-\eta^{2}}}-\int_{-1}^{\alpha} \frac{4 k^{2} \eta^{2} d \eta}{D} \\
& J_{2}=\frac{\varepsilon p_{x}}{\sqrt{\lambda}}\left[\int_{-1}^{\alpha} \frac{-8 k \eta \sqrt{k^{2} \eta^{2}+k^{\prime 2}} d \eta}{\sqrt{1-\eta^{2}}}+\int_{-1}^{\alpha} \frac{4 k \eta d \eta}{D}\right] \\
& J_{3}=\frac{\varepsilon^{2} p_{x}^{2}}{\lambda}\left[\int_{-1}^{\alpha} \frac{2 \sqrt{k^{2} \eta^{2}+k^{\prime 2}} d \eta}{\sqrt{1-\eta^{2}}}-\int_{-1}^{\alpha} \frac{d \eta}{D}\right] .
\end{aligned}
$$

Évaluons $J_{1}=J_{1}^{\prime}+J_{1}^{\prime \prime}$ où l'on a :

$$
\begin{aligned}
& J_{1}^{\prime}=\int_{-1}^{0} \frac{8 k^{2} \eta^{2} \sqrt{k^{2} \eta^{2}+k^{\prime 2}} d \eta}{\sqrt{1-\eta^{2}}}-\int_{-1}^{0} \frac{4 k^{2} \eta^{2} d \eta}{D} \\
& J_{1}^{\prime \prime}=\int_{0}^{\alpha} \frac{8 k^{2} \eta^{2} \sqrt{k^{2} \eta^{2}+k^{\prime 2}} d \eta}{\sqrt{1-\eta^{2}}}-\int_{0}^{\alpha} \frac{4 k^{2} \eta^{2} d \eta}{D}
\end{aligned}
$$

La quantité $J_{1}^{\prime}$ a été évaluée dans le cas plat, voir [1] et s'écrit :

$$
J_{1}^{\prime}=\frac{4}{3}\left[E\left(1-2 k^{\prime 2}\right)+k^{\prime 2} K\right]=\frac{4}{3}+o\left(k^{\prime}\right) .
$$

Par ailleurs, on a clairement :

$$
J_{1}^{\prime \prime}=O\left(\alpha^{3}\right)+o\left(k^{\prime}\right)
$$

et on peut donc écrire :

$$
J_{1}=\frac{4}{3}+O\left(\alpha^{3}\right)+o\left(k^{\prime}\right)
$$

Pour évaluer $J_{2}$ on écrit :

$$
J_{2}=-2 p_{x}\left[\frac{\varepsilon}{\sqrt{\lambda}} \int_{-1}^{\alpha}\left(\frac{4 k \eta \sqrt{k^{2} \eta^{2}+k^{\prime 2}}}{\sqrt{1-\eta^{2}}}-\frac{2 k \eta}{D}\right) d \eta\right]=-2 p_{x} I_{2}
$$

où $I_{2}$ a été évalué précédemment et l'on obtient donc :

$$
J_{2}=-2 p_{x}\left[\frac{\varepsilon}{\sqrt{\lambda}}\left(-2 \alpha+o\left(\alpha^{2}\right)+o\left(k^{\prime}\right)\right)\right]=\frac{4 \varepsilon \alpha}{\sqrt{\lambda}}+o\left(\alpha^{3}\right)+o\left(k^{\prime}\right) .
$$

Enfin on a :

$$
J_{3}=\frac{\varepsilon^{2} p_{x}^{2}}{\lambda} \int_{-1}^{\alpha} \frac{2 \sqrt{k^{2} \eta^{2}+k^{\prime 2}} d \eta}{\sqrt{1-\eta^{2}}}-\frac{\varepsilon^{2} p_{x}^{2}}{\lambda} \int_{-1}^{\alpha} \frac{d \eta}{D}=-\frac{\varepsilon^{2} r}{2 \sqrt{\lambda}}+\frac{2 \varepsilon^{2}}{\lambda}+o\left(\alpha^{2}\right)+o\left(k^{\prime}\right) .
$$

Soit donc :

$$
\lambda^{\frac{3}{2}} Z_{1}=\frac{4}{3}-\frac{\varepsilon^{2} r}{2 \sqrt{\lambda}}+o\left(\frac{1}{\sqrt{\lambda}}\right)+o\left(k^{\prime}\right)
$$


et l'on obtient :

$$
\begin{aligned}
Z_{1} & =\left(\frac{1}{\sqrt{\lambda}}\right)^{3}\left[\frac{4}{3}-\frac{1}{\sqrt{\lambda}} \frac{\varepsilon^{2} r}{2}+o\left(\frac{1}{\sqrt{\lambda}}\right)\right] \\
& =\left(\frac{X}{2}+\frac{\varepsilon^{2} r}{16} X^{2}\right)^{3}\left(\frac{4}{3}-\frac{\varepsilon^{2} r}{2}\left(\frac{X}{2}+\frac{\varepsilon^{2} r}{16} X^{2}\right)\right)+\ldots \\
& =\frac{X^{3}}{6}+\frac{\varepsilon^{2} r}{32} X^{4}+o\left(X^{4}\right) .
\end{aligned}
$$

On a donc calculé les deux premiers termes du développement asymptotiques. Le premier coefficient est identique $\grave{a}$ celui du cas plat. En poussant les calculs à $o\left(k^{\prime}\right)$ on calcule ainsi tous les coefficients de la partie analytique du graphe de $c_{3}: Z_{1}=u_{1}(X)+o\left(k^{\prime}\right)$. Le graphe n'est pas sous- analytique et le terme résiduel peut être évalué en calculant les coefficients $A, B, C$ et $\bar{A}, \bar{B}, \bar{C}$ dans la représentation :

$$
\begin{aligned}
& X=\frac{1}{\sqrt{\lambda}}\left(A_{1}\left(\frac{1}{\sqrt{\lambda}}\right)+A k^{\prime 2} \ln \frac{1}{k^{\prime}}+B k^{\prime 2} \ln _{2} \frac{1}{k^{\prime}}+C k^{\prime 2}+o\left(k^{\prime 2}\right)\right) \\
& Z_{1}=\frac{4}{3 \lambda^{\frac{3}{2}}}\left(A_{2}\left(\frac{1}{\sqrt{\lambda}}\right)+\bar{A} k^{\prime 2} \ln \frac{1}{k^{\prime}}+\bar{B} k^{\prime 2} \ln _{2} \frac{1}{k^{\prime}}+\bar{C} k^{\prime 2}+o\left(k^{\prime 2}\right)\right)
\end{aligned}
$$

et l'on obtient la proposition suivante.

Proposition 4.20. Pour $r$ assez petit, le graphe de $c_{3}$ n'est pas sous-analytique.

\subsubsection{Les courbes $c_{2}, c_{4}$ et $c_{5}$}

La courbe $c_{2}$ s'évalue comme la courbe $c_{3}$ en utilisant les formules intégrales. Pour évaluer les courbes $c_{4}$ et $c_{5}$ il est préférable d'utiliser la paramétrisation des géodésiques avec les fonctions de Jacobi. Faisons par exemple ce calcul pour la courbe $c_{4}$. Dans ce cas la variable $y$ se comporte comme un dn et à la seconde intersection on a :

$$
t=r, \quad r k \sqrt{\lambda}=2 K(\hat{k})
$$

où $K(\hat{k})$ est l'intégrale complète de module $\hat{k}$ défini par :

$$
\hat{k}^{2}=\sqrt{1-\hat{k}^{\prime 2}}, \quad \hat{k}^{\prime 2}=-\frac{k^{\prime \prime}}{k^{2}}
$$

et $k^{\prime \prime}$ est donné par :

$$
2 k^{\prime \prime}=1+p_{x}-\frac{\varepsilon^{2} p_{x}^{2}}{2 \lambda}
$$

Les formules de la proposition 4.10 se simplifient alors en :

$$
\begin{aligned}
& X_{2}=r \sin \varphi\left(1-\frac{3 \varepsilon^{2} \sin \varphi}{2 \lambda}+\frac{\varepsilon^{4} \sin ^{2} \varphi}{2 \lambda^{2}}\right)+\frac{4 k E(K)}{\sqrt{\lambda}}\left(1-\frac{\varepsilon^{2} \sin \varphi}{\lambda}\right) \\
& Z_{2}=\frac{r}{3 \lambda}\left(1-\sin ^{2} \varphi+\frac{5 \varepsilon^{2} \sin ^{3} \varphi}{2 \lambda}-\frac{\varepsilon^{4} \sin ^{4} \varphi}{\lambda^{2}}\right)+\frac{4 \sin \varphi k E(K)}{3 \lambda^{\frac{3}{2}}}\left(\frac{2 \varepsilon^{2} \sin \varphi}{\lambda}-1\right)
\end{aligned}
$$


où l'on a $p_{x}=\sin \varphi$ et l'on peut supposer $\left.\varphi \in\right]-\frac{\pi}{2},+\frac{\pi}{2}\left[\right.$. On doit calculer lorsque $\varphi \rightarrow-\frac{\pi}{2}$ et $p_{x}$ est donné lorsque $\lambda \rightarrow+\infty$ et $k^{\prime \prime} \rightarrow 0$ par

$$
p_{x}=\frac{\lambda}{\varepsilon^{2}}\left(1-\sqrt{1+\frac{2 \varepsilon^{2}}{\lambda}\left(1-2 k^{\prime \prime}\right)}\right)=-1+\frac{\varepsilon^{2}}{2 \lambda}+o\left(\frac{1}{\lambda}\right)+O\left(k^{\prime \prime}\right) .
$$

Soit donc:

$$
X_{2}=r\left(-1+\frac{\varepsilon^{2}}{2 \lambda}\right)\left(1+\frac{3 \varepsilon^{2}}{2 \lambda}\right)+\frac{4}{\sqrt{\lambda}}+o\left(\frac{1}{\lambda}\right)+o\left(k^{\prime \prime}\right)
$$

En simplifiant on obtient :

$$
X_{2}=-r+\frac{4}{\sqrt{\lambda}}-\frac{r \varepsilon^{2}}{\lambda}+o\left(\frac{1}{\lambda}\right)+o\left(k^{\prime \prime}\right)
$$

et en posant : $X=\frac{X_{2}+r}{2}$ il vient :

$$
X=\frac{2}{\sqrt{\lambda}}-\frac{r}{2} \frac{\varepsilon^{2}}{\lambda}+o\left(\frac{1}{\lambda}\right)
$$

En résolvant on obtient :

$$
\frac{1}{\sqrt{\lambda}}=\frac{X}{2}+\frac{r \varepsilon^{2}}{16} X^{2}+o\left(X^{2}\right)
$$

Par ailleurs :

$$
Z_{2}=\frac{r}{3 \lambda}\left(1-\left(1-\frac{\varepsilon^{2}}{\lambda}\right)-\frac{5 \varepsilon^{2}}{2 \lambda}+o\left(\frac{1}{\lambda}\right)\right)+\frac{4}{3 \lambda^{\frac{3}{2}}}\left(1+o\left(\frac{1}{\sqrt{\lambda}}\right)\right)+o\left(k^{\prime \prime}\right)
$$

et après simplification on obtient :

$$
Z_{2}=\frac{4}{3 \lambda^{\frac{3}{2}}}-\frac{r \varepsilon^{2}}{2 \lambda^{2}}+o\left(\frac{1}{\lambda^{2}}\right)=\frac{4}{3}\left(\frac{X}{2}+\frac{r \varepsilon^{2}}{16} X^{2}\right)^{3}-\frac{r \varepsilon^{2}}{2}\left(\frac{X}{2}+\frac{r \varepsilon^{2}}{16}\right)^{4}+o\left(X^{4}\right) .
$$

Soit la formule :

$$
Z_{2}=\frac{X^{3}}{6}+\frac{r \varepsilon^{2}}{32} X^{4}+o\left(X^{4}\right)
$$

On observe que les deux premiers termes de $c_{3}$ et $c_{4}$ coincident et il faut calculer un terme supplémentaire pour séparer les courbes. Des calculs plus précis donnent le résultat suivant.

Proposition 4.21. Au voisinage du point $(-r, 0)$ les courbes $c_{3}$ et $c_{4}$ sont des graphes donnés respectivement par les formules:

- $c_{3}: z=\frac{1}{6} X^{3}+\frac{r \varepsilon^{2}}{32} X^{4}+\frac{\varepsilon^{2}}{32}\left(\frac{r^{2} \varepsilon^{2}}{4}-3\right) X^{5}+o\left(X^{5}\right)$

- $c_{4}: z=\frac{1}{6} X^{3}+\frac{r \varepsilon^{2}}{32} X^{4}+\frac{\varepsilon^{2}}{32}\left(\frac{9 r^{2} \varepsilon^{2}}{4}-2\right) X^{5}+o\left(X^{5}\right)$

où $X=\frac{x+r}{2}$. 


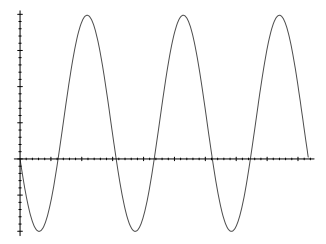

FIGURE 29

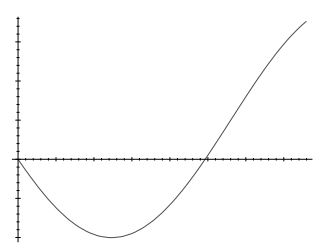

FIGURE 30

\subsection{Remarques}

On a donc décrit les deux algorithmes utilisables pour évaluer les développements asymptotiques des branches du front d'onde se ramifiant en $(-r, 0)$ : les formules intégrales pour $c_{2}, c_{3}$ et les fonctions de Jacobi pour $c_{4}, c_{5}$. Si le rayon $r$ est assez petit aucune de ces branches n'est sous-analytique. Par contre on observe que la transcendance est très différente. Les courbes $c_{2}, c_{3}$ calculées à la première intersection avec $y=0$ ont dans leurs représentations des doubles logarithmes, par contre pour les courbes $c_{4}, c_{5}$ la situation est similaire au cas plat et une simple singularité logarithmique est alors suffisante.

\subsection{Conjectures sur les points conjugués et de coupures}

Des simulations numériques conduisent aux conjectures suivantes sur la localisation des points conjugués et de coupures (comparer avec le cas plat, voir [1]). Elles ont été effectuées à l'aide d'un intégrateur classique du type de Runge-Kutta d'ordre 5(4) complété par dense-output d'odre 4 (voir [11] pour plus de précisions).

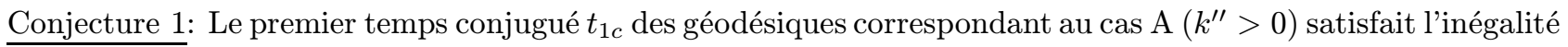

$$
\begin{array}{ll}
p_{x} \neq 1: & t_{1}<t_{1 c}<t_{2} \\
p_{x}=1: & t_{1}=t_{1 c}
\end{array}
$$

où $t_{1}, t_{2}$ sont les premier et deuxième temps d'intersection de la géodésique avec la surface de Martinet $y=0$.

Les figures 29 et 30 illustre cette conjecture. La projection sur le plan $(x, y)$ d'une géodésique du cas $\mathrm{A}$ correspondant à $p_{x} \neq 1$ est dessinée sur la figure 29, la figure 30 représentant cette même géodésique jusqu'à son premier point conjugué.

Conjecture 2: Le premier temps conjugué $t_{1 c}$ des géodésiques correspondant au cas B $\left(k^{\prime \prime}<0\right)$ satisfait l'inégalité

$$
\begin{array}{ll}
p_{x} \neq-1: & t_{2}<t_{1 c} \\
p_{x}=-1: & t_{2}=t_{1 c} .
\end{array}
$$




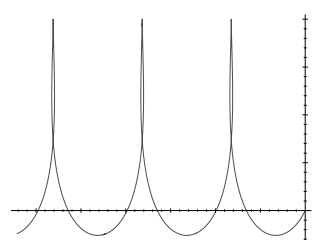

FiGURE 31

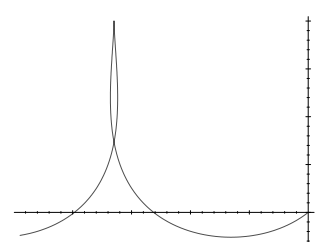

FigURE 32

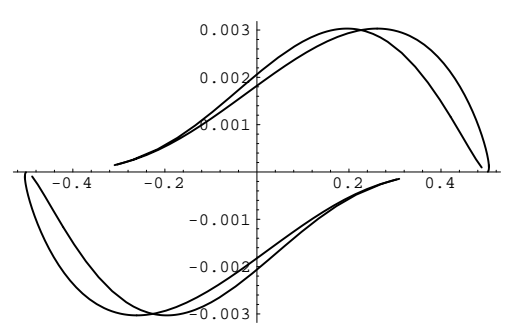

FiguRE 33

La figure 31 représente la projection sur le plan $(x, y)$ d'une géodésique du cas B telle que $p_{x} \neq-1$ et elle est dessinée jusqu'a son premier point conjugué en figure 32 .

Conjecture 3: Un point de coupure pour une géodésique du type $\mathrm{B}$ associée à $p_{x} \neq-1$ correspond exactement à la deuxième intersection de la géodésique avec $y=0$.

\subsection{Trace de la sphère $S(0, r)$ de petit rayon avec le plan de Martinet}

En tenant compte des conjectures précédentes, des simulations numériques donnant les positions respectives de $\Gamma_{1}^{+}$et $\Gamma_{1}^{-}$représentées sur la figure 33 (réalisée avec le package des fonctions elliptiques de Mathematica) et les calculs asymptotiques précédents on peut conjecturer que la trace de la sphère de petit rayon avec le plan de Martinet est représentée sur la figure 34.

On observe qu'une branche du lieu de coupure correspondant à la courbe $c_{4}$, se terminant en $(-r, 0)$ reste contenue dans le plan $y=0$. Son autre extrémité est un point conjugué correspondant à $p_{x}=-1$. 


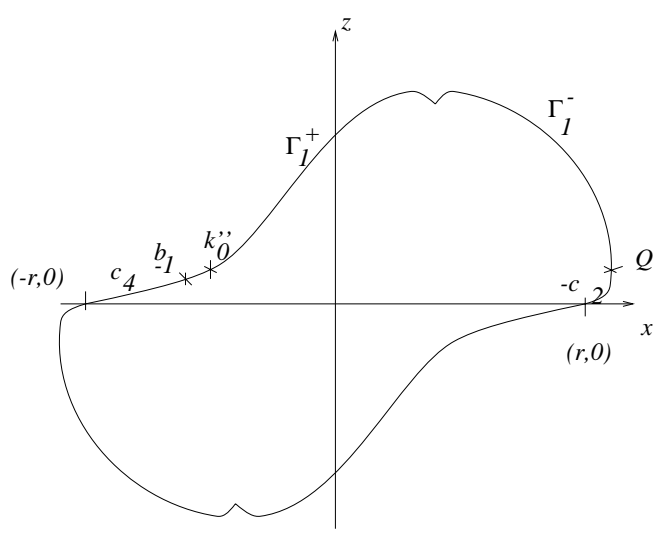

FIGURE 34

\subsection{Conclusion : Le rôle du paramètre $\alpha$}

Notre étude montre que le rôle du paramètre $\alpha$ est de faire que les trajectoires en rotation du pendule correspondent à des projections de géodésiques issues de 0 . Cette propriété est source d'insabilité par rapport au cas plat : phénomène de doublement de période et phénomène de doublement du logarithme.

\section{5. Étude De la PERTURbation À Un PARAmètre $g=d x^{2}+(1+\beta x)^{2} d y^{2}$}

\subsection{Préliminaires}

L'objectif de ce paragraphe est d'analyser le rôle du paramètre $\beta$ dans la forme normale sur le comportement des géodésiques et la nature de la sphère sous-riemannienne. L'étude est loin d'être complète et ne constitue qu'une introduction au cas non intégrable qui sera traité dans un article ultérieur [10]. La perturbation à un paramètre $g=d x^{2}+(1+\beta x)^{2} d y^{2}$ possède les propriétés suivantes :

1. La courbure de Gauss de la métrique projetée $g_{R}$ est strictement positive si $\beta$ est non nul (cf. Sect. 2.5.3).

2. La géodésique anormale $a: t \mapsto( \pm t, 0,0)$ n'est pas strictement anormale.

3. L'équation des géodésiques normales n'est pas intégrable par quadratures.

\subsection{Symétrie et optimalité}

On peut par des considérations géométriques élémentaires obtenir des renseignements importants sur le statut d'optimalité des géodésiques. Pour la géodésique anormale la propriété de rigidité géodésique n'est pas évidente mais une conséquence de la non stricte anormalité est la suivante.

Lemme 5.1. Il existe $M>0$ tel que si $L(a) \leq M$ alors a(.) est globalement minimisante.

Par ailleurs on observe que la géométrie SR: $(D, g)$ où $D=\operatorname{ker} \omega, \omega=d z-\frac{y^{2}}{2} d x$ et $g=d x^{2}+(1+\beta x)^{2} d y^{2}$ possède les deux symétries suivantes :

$$
S:(x, y, z, \beta) \mapsto(-x, y,-z,-\beta), S^{\prime}:(x, y, z) \mapsto(x,-y, z) .
$$

Ces deux symétries ont des conséquences importantes. En notant $p_{z}=\lambda$, on peut dans notre étude supposer : $\left(H_{1}\right) \lambda \geq 0$ car le cas $\lambda \leq 0$ s'obtient en changeant $(-x,-z,-\beta)$ en $(x,-z,-\beta)$. La seconde conséquence justifie le lemme suivant. 
Lemme 5.2. Soit e $:[0, T] \mapsto(x(t), y(t), z(t))$ une géodésique normale telle que e $(0)=0$ et Ime n'appartient pas à Ox. Alors e n'est pas minimisante au delà de sa première intersection avec le plan de Martinet $y=0$.

Preuve. Du fait de la symétrie $S^{\prime}$, à sa première intersection avec $y=0, e$ rencontre au même point et avec la même longueur la géodésique $\tilde{e}(t)=(\tilde{x}(t),-\tilde{y}(t), \tilde{z}(t))=(-x(t), y(t),-z(t))$ qui est distincte de $e($.$) si \dot{y}(0) \neq 0$, c'est-à-dire si $y(t)$ n'est pas identiquement nul.

\subsection{Notations}

Les géodésiques normales vérifient les équations (2.3) et en introduisant le paramètre $\sqrt{c} \frac{d}{d t}=\frac{d}{d \tau}$ où $c=$ $(1+\beta x)^{2}$, la projection des géodésiques normales dans le plan $(y, \theta)$ est décrite par le système :

$$
\frac{d y}{d \tau}=\sin \theta, \frac{d \theta}{d \tau}=-(y \lambda+\beta \sin \theta)
$$

qui est équivalente à l'équation du second ordre :

$$
\theta^{\prime \prime}+\lambda \sin \theta+\beta \cos \theta \theta^{\prime}=0
$$

où $\theta^{\prime}$ est la dérivée par rapport à $\tau$. D'après (5.1) le plan $y=0$ induit la section :

$$
\theta^{\prime}=-\beta \sin \theta
$$

Dans le problème SR au voisinage des géodésiques anormales qui se projettent ici en $\theta=k \pi$, on s'intéresse aux solutions telles que $|\lambda|$ est grand.

En utilisant $\left(H_{1}\right): \lambda \geq 0$, on fera l'hypothèse :

$$
\left(H_{2}\right): \lambda \gg|\beta|,
$$

et l'équation précédente s'écrit alors avec $s=\sqrt{\lambda} \tau$ et $\varepsilon=\frac{\beta}{\sqrt{\lambda}}$ petit paramètre sous la forme :

$$
\frac{d^{2} \theta}{d s^{2}}+\sin \theta+\varepsilon \cos \theta \frac{d \theta}{d s}=0
$$

et la contrainte (5.3) devient :

$$
\frac{d \theta}{d s}=-\varepsilon \sin \theta
$$

L'équation (5.4) est une perturbation de l'équation $d u$ pendule où le terme $\varepsilon \cos \theta \frac{d \theta}{d s}$ est un terme de dissipation d'énergie. Le premier travail est de comprendre le rôle de ce terme sur les géodésiques normales.

\subsection{Portrait de phase de l'équation (5.2) avec $\lambda>>|\beta|, \beta \neq 0$}

Lemme 5.3. Soit $\theta:[a, b] \rightarrow \mathbf{R}$ une solution de l'équation (5.2). On a la formule de balance d'énergie (E) suivante :

$$
\begin{aligned}
\frac{\theta^{\prime 2}(b)-\theta^{\prime 2}(a)}{2} & +\lambda \beta \int_{a}^{b} \sin ^{2} \theta(\tau) d \tau=\lambda(\cos \theta(b)-\cos \theta(a)) \\
& +\frac{\beta^{2}}{4}(\cos 2 \theta(b)-\cos 2 \theta(a))+\beta\left(\sin \theta(a) \theta^{\prime}(a)-\sin \theta(b) \theta^{\prime}(b)\right) .
\end{aligned}
$$


Preuve. D'après (5.2) on a :

$$
\theta^{\prime \prime} \theta^{\prime}+\beta \cos \theta \theta^{\prime 2}+\lambda \sin \theta \theta^{\prime}=0
$$

et en intégrant entre $a$ et $b$ il vient :

$$
\left[\frac{1}{2} \theta^{\prime 2}\right]_{a}^{b}+\beta \int_{a}^{b} \cos \theta \theta^{\prime 2} d \tau-[\lambda \cos \theta]_{a}^{b}=0
$$

De plus :

$$
\beta \int_{a}^{b} \cos \theta \theta^{\prime 2} d \tau=\beta \int_{a}^{b}(\sin \theta)^{\prime} \theta^{\prime} d \tau
$$

et en intégrant par parties on obtient :

$$
\beta \int_{a}^{b} \cos \theta \theta^{\prime 2} d \tau=\beta\left[\sin \theta \theta^{\prime}\right]_{a}^{b}-\beta \int_{a}^{b} \sin \theta \theta^{\prime \prime} d \tau
$$

et en utilisant l'équation (5.2) il vient :

$$
\begin{aligned}
-\beta \int_{a}^{b} \sin \theta \theta^{\prime \prime} d \tau & =\beta \int_{a}^{b} \sin \theta\left(\lambda \sin \theta+\beta \cos \theta \theta^{\prime}\right) d \tau \\
& =\lambda \beta \int_{a}^{b} \sin ^{2} \theta d \tau+\frac{\beta^{2}}{2} \int_{a}^{b} \sin 2 \theta \theta^{\prime} d \tau=\lambda \beta \int_{a}^{b} \sin ^{2} \theta d \tau-\frac{\beta^{2}}{4}[\cos 2 \theta]_{a}^{b}
\end{aligned}
$$

La formule (E) est donc prouvée.

\subsection{Propriétés}

L'équation (5.2): $\theta^{\prime \prime}+\lambda \sin \theta+\beta \cos \theta \theta^{\prime}=0$ avec $\lambda>>|\beta|$ et $\beta \neq 0$ est une gerbe sur $S^{1}$ muni de la coordonnée $Z=\mathrm{e}^{i \theta}$ et le système

$$
\theta^{\prime}=v, \quad v^{\prime}=-\lambda \sin \theta-\beta \cos \theta v
$$

définit un champ de vecteurs $\Xi$ sur le cylindre $S^{1} \times \mathbf{R}$. Il possède deux positions d'équilibre : $M_{1}=(1,0)$ et $M_{2}=(-1,0)$ qui correspondent à $\theta=k \pi$ et $\theta^{\prime}=0$ et qui représentent la projection de la géodésique anormale. Ces points singuliers se classifient ainsi :

- Le point $M_{1}=(1,0)$ : les deux valeurs propres du linéarisé sont $\sigma=\frac{-\beta \pm \sqrt{\beta^{2}-4 \lambda}}{2}$ et $M_{1}$ est donc un foyer stable (resp. instable) si $\beta>0$ (resp. $\beta<0$ ).

- Le point $M_{2}=(-1,0)$ : les deux valeurs propres du linéarisé sont $\sigma=\frac{\beta \pm \sqrt{\beta^{2}+4 \lambda}}{2}$ et $M_{2}$ est donc un point col.

Lemme 5.4. Le champ de vecteurs $\Xi$ est complet.

Preuve. Soit $\theta$ : $] e_{-}, e_{+}\left[\rightarrow \mathbf{R}\right.$ une solution maximale de (5.2) et supposons par exemple $e_{+}<+\infty$. Alors $\theta^{\prime}(t) \rightarrow \infty$ quand $t \rightarrow e_{+}$et $\forall M>0$ il existe $\left.b_{0} \in\right] e_{-}, e_{+}[$tel que :

$$
\left|\theta^{\prime}(b)\right| \geq 4 M \text { si } b \geq b_{0} .
$$


En particulier si $M \geq 1$, on a :

$$
\frac{\theta^{\prime} 2(b)}{2}>M\left(\left|\theta^{\prime}(b)\right|+1\right)
$$

Soit $e_{-}<a<b<e_{+}$et appliquons la formule (E) de balance d'énergie. On obtient

$$
\frac{\theta^{\prime 2}}{2}(b) \leq|\lambda \beta|\left(e_{+}-a\right)+2 \lambda+\frac{\beta^{2}}{2}+|\beta|\left(\left|\theta^{\prime}(a)\right|+\left|\theta^{\prime}(b)\right|\right)+\frac{\theta^{\prime 2}(a)}{2} .
$$

Donc si

$$
M \geq \max \left(|\beta|,|\lambda \beta|\left(e_{+}-a\right)+2 \lambda+\frac{\beta^{2}}{2}+|\beta|\left|\theta^{\prime}(a)\right|+\frac{\theta^{\prime 2}(a)}{2}\right)
$$

on a

$$
\frac{\theta^{\prime} 2(b)}{2} \leq M\left(\left|\theta^{\prime}(b)\right|+1\right)
$$

Cela contredit (5.7). La preuve est similaire si $e_{-}>-\infty$.

Lemme 5.5. Les seules trajectoires périodiques de $\Xi$ sont les positions d'équilibre $(1,0)$ et $(-1,0)(\beta \neq 0)$.

Preuve. Soit $\theta: \mathbf{R} \rightarrow \mathbf{R}$ une solution de (5.2) telle que la trajectoire associée $\gamma$ de $\Xi$ sur le cylindre soit une trajectoire périodique. On doit considérer deux cas :

- Cas $a: \gamma$ est non homotope à 0 . Alors il existe $a, b \in \mathbf{R}, a<b$ tels que $\theta(a)=0, \theta(b)=2 \pi \mathbf{Z}$ et $\theta^{\prime}(a)=\theta^{\prime}(b)$. En appliquant à $\theta:[a, b] \rightarrow \mathbf{R}$ la formule de balance d'énergie on a :

$$
\lambda \beta \int_{a}^{b} \sin ^{2} \theta(\tau) d \tau=0
$$

Puisque $\lambda \beta \neq 0$, on obtient $\sin ^{2} \theta(\tau)=0$, pour tout $\tau \in[a, b]$. Donc $\theta(\tau)=k \pi, k \in \mathbf{Z}$ pour tout $\tau$.

- Cas b: $\gamma$ est homotope à 0 . On observe que $\Xi$ admet deux points singuliers $(1,0)$ et $(-1,0)$ d'indices respectifs +1 et -1 . D'après le théorème de Poincaré, $(1,0)$ est à l'intérieur du chemin fermé $\gamma$. Il existe donc $a<b$ tel que $\theta(a)=\theta(b)=0$ et $\theta^{\prime}(a)=\theta^{\prime}(b)$. En appliquant à $\theta$ la formule de balance d'énergie, on obtient encore $\int_{a}^{b} \sin ^{2} \theta(\tau) d \tau=0$ et donc $\theta(\tau)=k \pi$.

Lemme 5.6. Le champ de vecteurs $\Xi$ n'a pas de séparatrices connectant les cols $(\beta \neq 0)$.

Preuve. Sur le cylindre le seul col est $M_{2}=(-1,0)$. Supposons que $\theta: \mathbf{R} \rightarrow \mathbf{R}$ soit une connection de cols, alors : $\lim _{\tau \rightarrow \pm \infty} \exp (i \theta(\tau))=-1, \lim _{\tau \rightarrow \pm \infty} \theta^{\prime}(\tau)=0$. Soit $a<b$ et appliquons la formule de balance d'énergie avec $a \rightarrow-\infty$ et $b \rightarrow+\infty$, on obtient $\int_{-\infty}^{+\infty} \sin ^{2} \theta(\tau) d \tau=0$, ce qui est absurde.

\subsection{Portrait de phase et application $T$}

En utilisant l'analyse précédente et le théorème de Poincaré-Bendixon (voir [32]) sur le cylindre on peut représenter le portrait de phase. On va supposer $\beta>0$, le cas $\beta<0$ étant similaire.

On observe que le système est invariant pour la symétrie $S^{\prime \prime}:\left(\theta, \theta^{\prime}\right) \rightarrow\left(-\theta,-\theta^{\prime}\right)$. On peut donc supposer $\theta^{\prime} \geq 0$. Toute trajectoire exceptée la séparatrice $(\Sigma)$ convergeant vers le point col, tend lorsque $t \rightarrow+\infty$, vers le foyer stable $M_{1}$. Donc l'ensemble $\omega$-limite de chaque trajectoire est soit le foyer soit le point col. De même l'ensemble $\alpha$-limite de chaque trajectoire est soit vide, soit le point col, le comportement asymptotique lorsque 


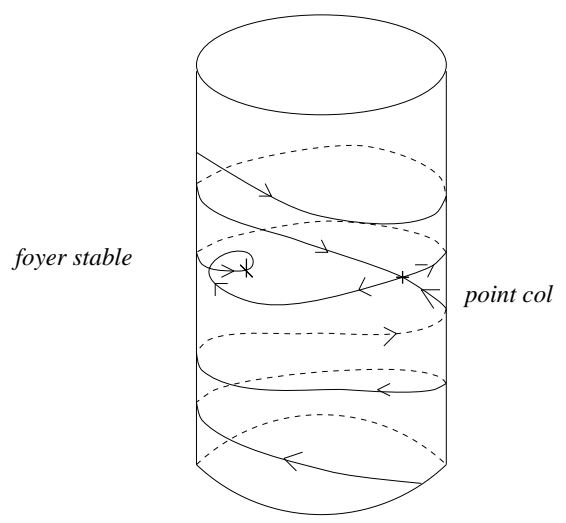

FIGURE 35

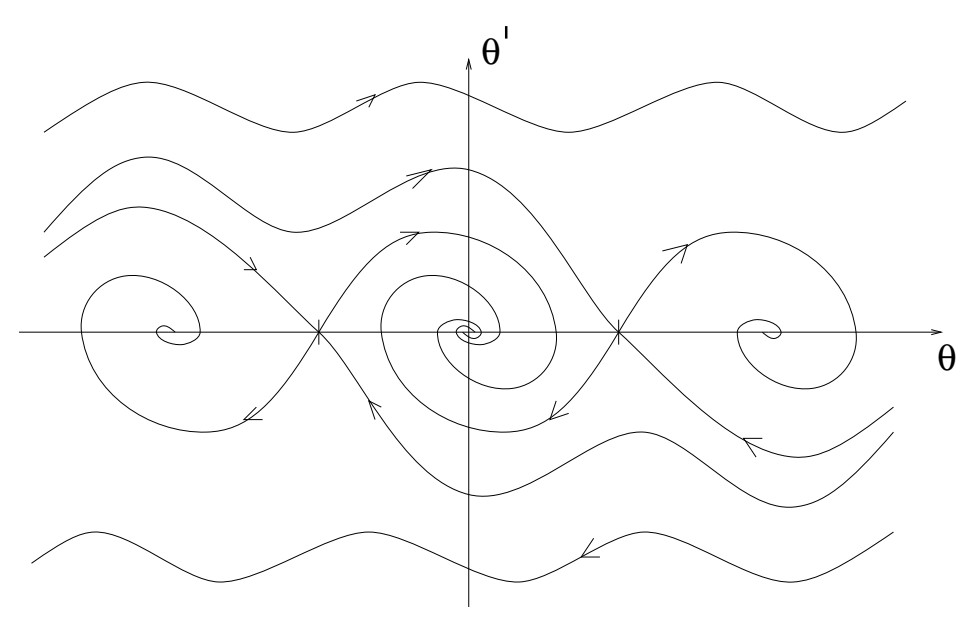

FiguRE 36

$t \rightarrow-\infty$ est compliqué à cause de la nature de l'amortissement. (Il n'y a pas de compactification évidente du champ de vecteurs $\Xi$ ). On représente sur la figure 35 (resp. Fig. 36) le portrait de phase sur le cylindre (resp. l'espace des phases).

La propriété fondamentale est la décroissance de l'application retour $T$ qui associe à un point de l'axe $\theta=-\pi$ la première intersection de la trajectoire avec l'axe $\theta=\pi$, voir figure 37 .

Cette application n'est définie que pour $\theta^{\prime} \geq \theta_{\Sigma}^{\prime}$ où $\theta_{\Sigma}^{\prime}$ est la condition initiale associée à la séparatrice $\Sigma$.

Une propriété intéressante du feuilletage est d'admettre un modèle topologique intégrable, [34]. En effet le portrait de phase est identique à celui du système :

$$
\ddot{x}+k \dot{x}|\dot{x}|+\omega^{2} \sin x=0
$$

où $k>0$ est le coefficient de frottement. 


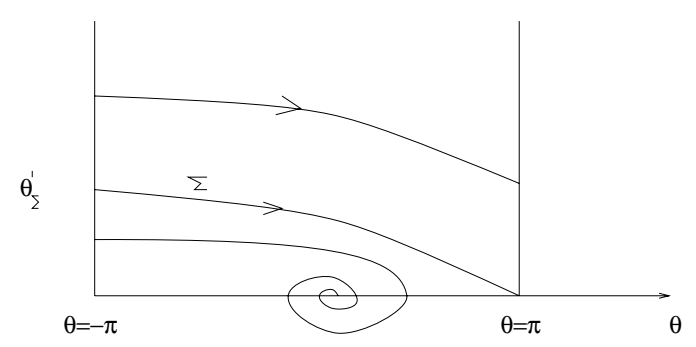

FIGURE 37
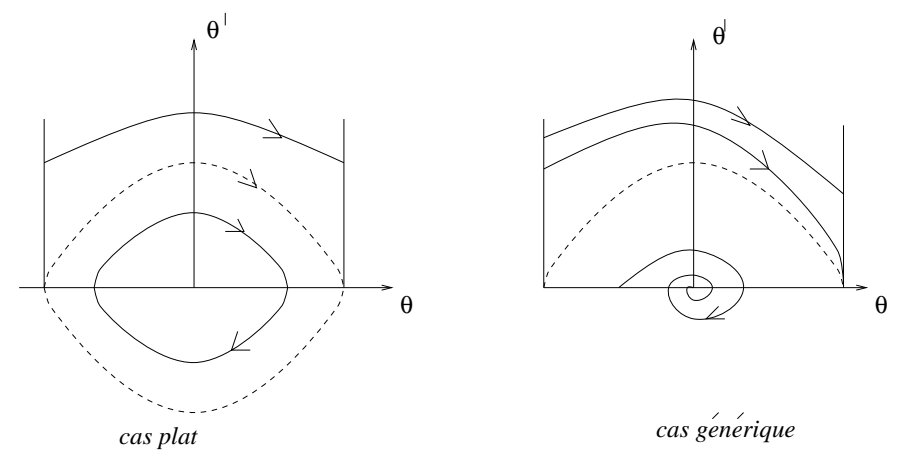

FIGURE 38

Les solutions sont également symétriques pour la symétrie $S^{\prime \prime}:(x, \dot{x}) \mapsto(-x,-\dot{x})$ et dans chaque domaine $\dot{x}>0$ où $\dot{x}<0$ on intègre aisément en écrivant avec $y=\dot{x}:$

$$
\frac{d y}{d x}=-\frac{\omega^{2} \sin x+k y|y|}{y}
$$

qui s'écrit aussi :

$$
\frac{d^{2} y}{d x^{2}} \pm 2 k y^{2}=-2 \omega^{2} \sin x
$$

et dont l'intégration élémentaire donne :

$$
y^{2}=C \exp ^{-2( \pm k x)}+\frac{2 \omega^{2}}{1+4 k^{2}} \cos x-\frac{4 \omega^{2}( \pm k)}{1+4 k^{2}} \sin x
$$

On construit donc la solution en recollant sur l'axe $y=0$ les deux intégrales premières.

\subsection{Stabilité}

L'étude précédente montre que si dans la forme normale graduée d'ordre $0: g=(1+\alpha y)^{2} d x^{2}+(1+\beta x+$ $\gamma y)^{2} d y^{2}$ le paramètre $\beta$ est non nul alors la projection des géodésiques normales sur le cylindre est non bornée pour $\lambda \gg \alpha, \beta, \gamma$, même au voisinage de la connection de selles du cas plat, $c f$. la figure 38 .

Ce résultat nous prouve que le développement des solutions calculé dans le paragraphe 2.5 est en général seulement formel (En fait notre analyse montre qu'il n'est convergent que lorsque $\alpha=\beta=0$.) Ce résultat nous dit en particulier que le front d'onde du cas plat est très instable. 


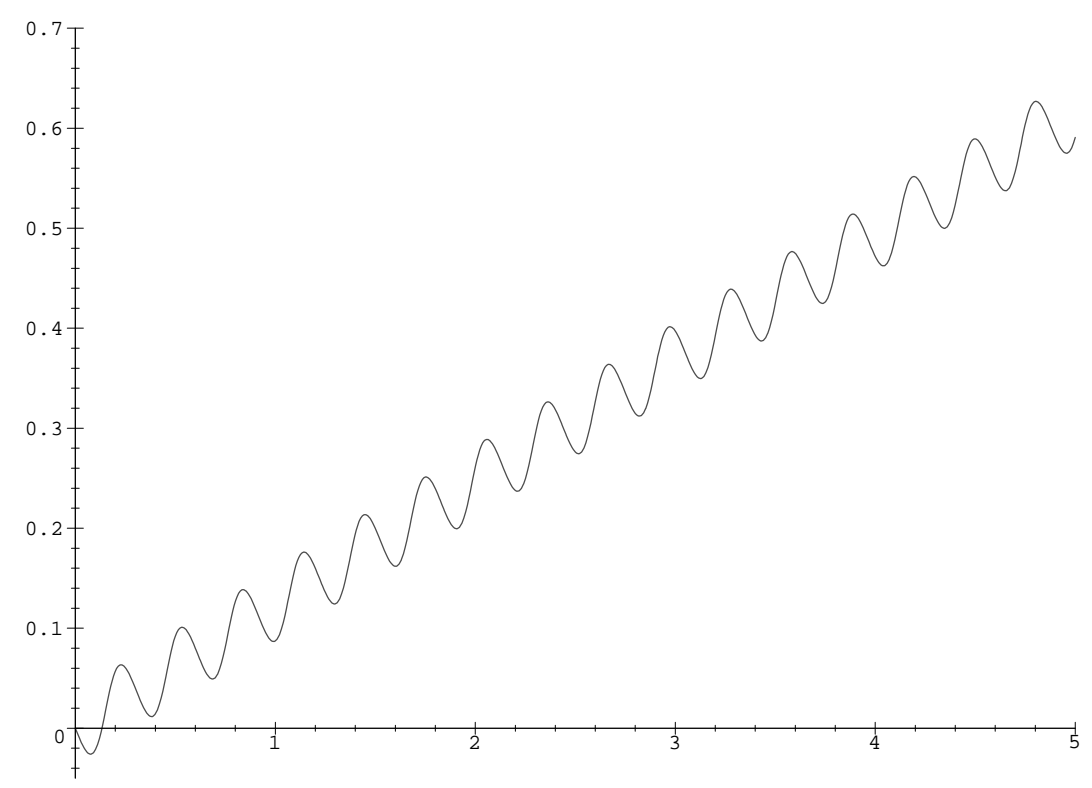

FIGURE 39

Pour la sphère, la situation n'est pas claire car elle est construite à l'aide de l'application premier retour.

\subsection{Représentation numérique des géodésiques}

Sur les figures 39-44 on a représenté les simulations numériques obtenues en calculant les composantes d'une géodésique, en comparant le cas plat et le cas dissipatif. On constate en particulier la variation d'amplitude après chaque oscillation de la coordonnée $y$ dans le cas dissipatif. Ce type de simulation indique bien comment modifier les développements de la section 2.5 pour tenir compte de certains effets séculaires : il faut moduler l'amplitude et la phase de la fonction cn. La figure 45 représente la sphère pour le système perturbé. Elle est numériquement semblable à celle du cas plat.

\subsection{Quelques remarques au sujet du calcul de la sphère $S(0, r)$ de petit rayon}

\subsubsection{Préliminaires}

Le problème important dans notre étude est d'évaluer la sphère $S(0, r)$ de petit rayon au voisinage du point $(-r, 0,0)$ extrémité de la géodésique anormale $a: t \mapsto(-t, 0,0)$ de longueur $r$ et qui appartient à la sphère si $r$ est assez petit car $a($.$) est minimisante. En particulier on s'intéresse aux branches de S(0, r) \cap\{y=0\}$ se ramifiant au point $(-r, 0)$. Il résulte du lemme 5.2 que ces branches sont contenues dans l'image de l'application premier retour car une géodésique distincte de $a($.$) n'est pas minimisante au delà de sa première intersection.$ Pour calculer ces branches on doit donc utiliser :

- Le feuilletage (5.4)

$$
\frac{d^{2} \theta}{d s^{2}}+\sin \theta+\varepsilon \cos \theta \frac{d \theta}{d s}=0
$$

- La contrainte (5.5) représentant le plan $y=0$ dans l'espace $(\theta, \dot{\theta})$ :

$$
\frac{d \theta}{d s}=-\varepsilon \sin \theta
$$




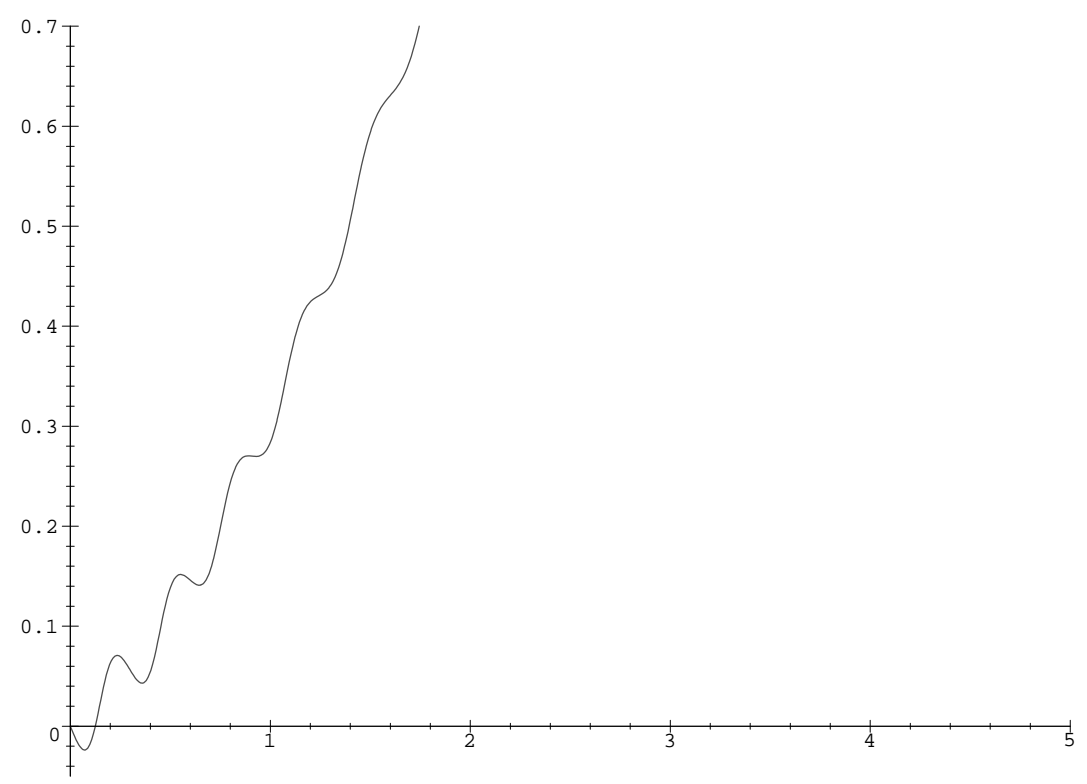

Figure 40. Courbe $t \rightarrow x(t)$.

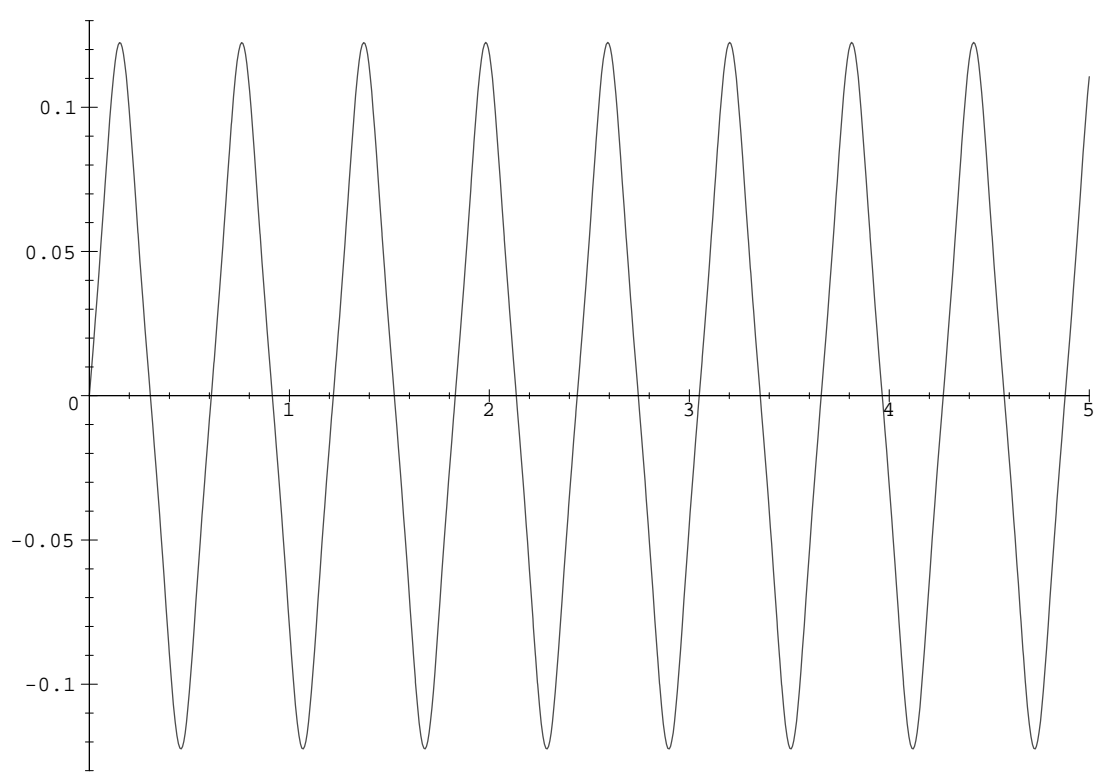

FigURE 41

- Fixer la longueur des géodésiques à $t=r$.

On a représenté sur la figure 46 l'application retour dans le plan $(\theta, \dot{\theta})$ associée au feuilletage (5.4) et à la section (5.5), au voisinage de la séparatrice $\Sigma$. En particulier l'existence de la séparatrice $\Sigma$ montre que l'application premier retour n'est pas propre. Dans le cas plat l'application premier retour dans le plan $(\theta, \dot{\theta})$ est simple et coincide avec $R_{\theta}: \theta \rightarrow-\theta$. La trace de la sphère avec $y=0$ coincide avec un graphe de la forme $: z=F\left(X, \frac{\mathrm{e}^{-\frac{1}{X}}}{X^{2}}\right)$ où $X=\frac{x+r}{2 r}$ où $F$ est un germe de fonctions analytiques. Ce type de modèle est similaire à celui obtenu en 


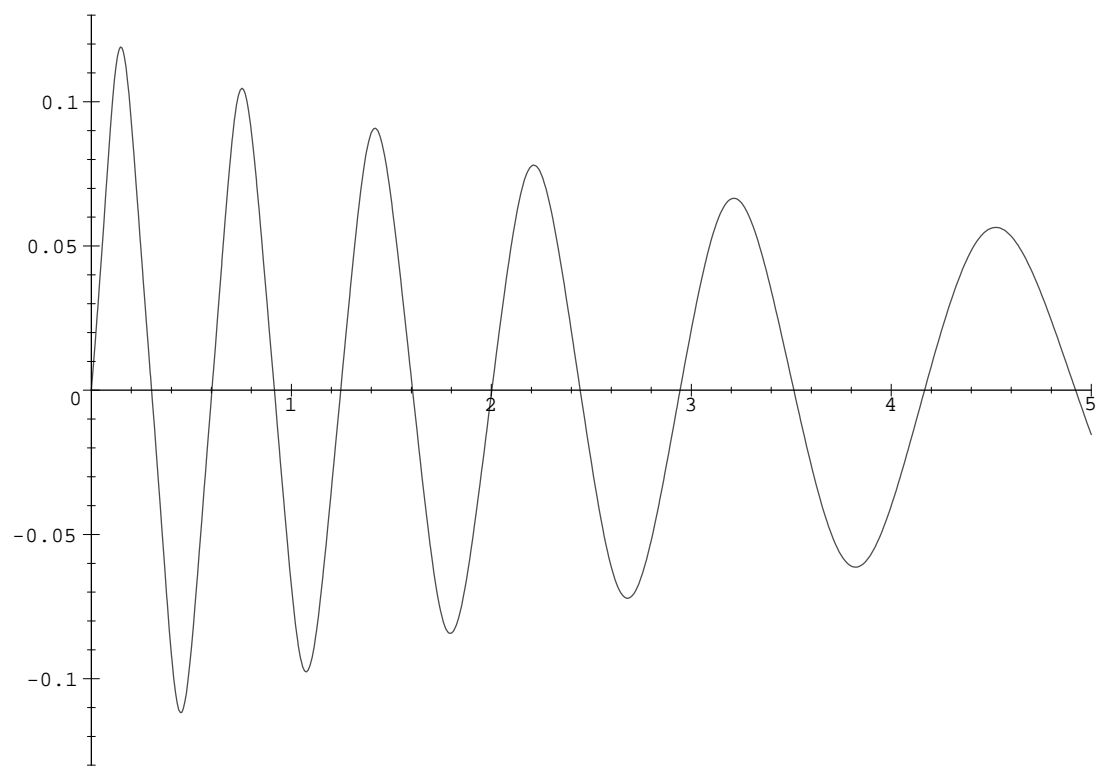

Figure 42. Courbe $t \rightarrow y(t)$.

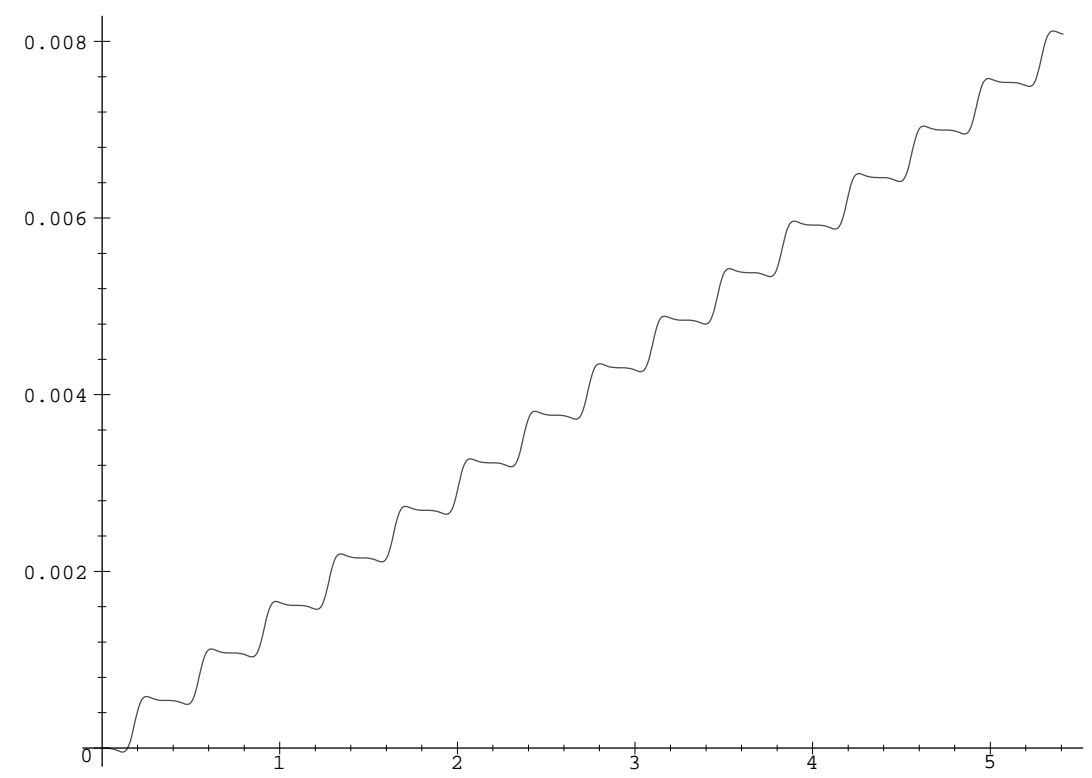

FIGURE 43

évaluant l'application de Poincaré-Dulac au voisinage d'un polycycle de sommets $\left(a_{1}, \ldots, a_{k}\right)$ où chaque sommet est un point col admettant un facteur intégrant analytique, voir [30]. Pour comprendre notre application retour, il est donc nécessaire d'examiner le passage du col dans notre feuilletage.

\subsubsection{Le passage du col}

Definition 5.7. Soit $X$ un champ de vecteurs analytique sur un voisinage de 0 dans $\mathbf{R}^{2}$ et admettant 0 comme point singulier. On dit que 0 est un col non dégénéré si le spectre de $\frac{\partial X}{\partial x}(0)$ est formé de deux valeurs propres 
B. BONNARD ET M. CHYBA

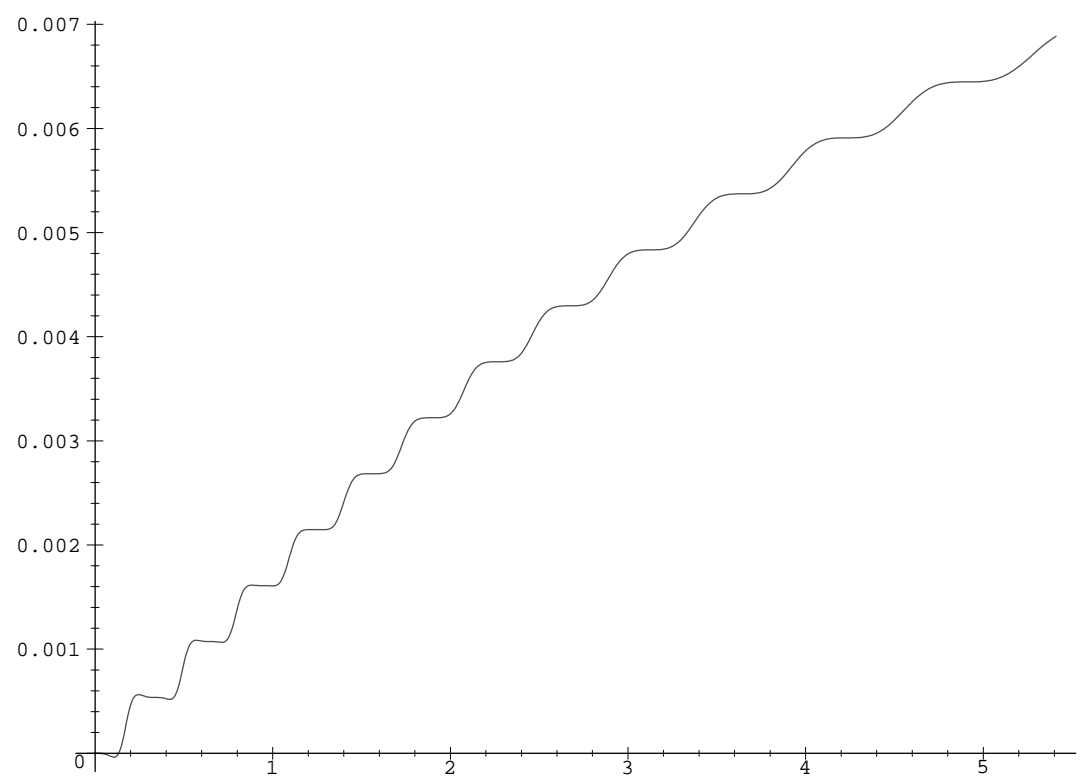

Figure 44. Courbe $t \rightarrow z(t)$.

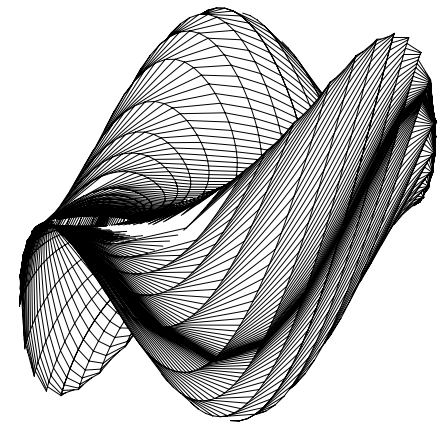

FiguRE 45

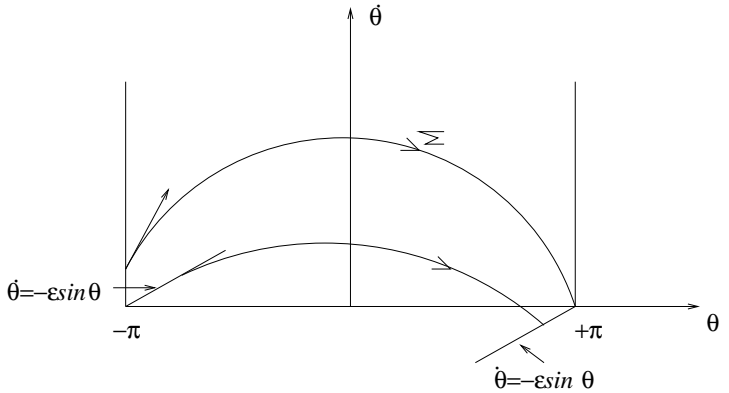

Figure 46 


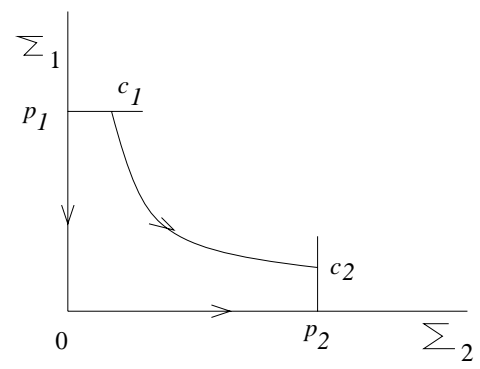

FIGURE 47

$\sigma_{1}, \sigma_{2}$ réelles avec $\sigma_{1} \sigma_{2}<0$. Le point 0 est alors formé de 4 secteurs cols et l'on choisit un des secteurs $S$ bordé par une séparatrice stable $\Sigma_{1}$ et une séparatrice instable $\Sigma_{2}$. Soit $c_{i}, i=1,2$ une courbe analytique lisse qui coupe transversalement $\Sigma_{i}$ en un point $p_{i}$ assez voisin de 0 . On note $T$ l'application de Poincaré-Dulac évaluée sur $c_{1}, c_{2}$, voir figure 47 .

On dit que $T$ est 1-pfaffienne si son graphe dans $\left(\mathbf{R}^{2}, 0\right)$ est le germe en 0 d'une courbe intégrale d'une 1-forme $\eta=a(x) d x_{1}+b(x) d x_{2}$ à singularité 0 algébriquement isolée. On dit que le point col 0 est intégrable ou admet un facteur intégrant analytique si la 1-forme $\omega=X_{2}(x) d x_{1}-X_{1}(x) d x_{2}$ associée à $X$ possède un germe $I$ en 0 de fonction analytique telle que $\frac{\omega}{I}$ soit fermée.

Le résultat fondamental que l'on va utiliser est le résultat de [30], voir aussi [31].

Théorème 5.8. L'application $T$ de Poincaré-Dulac est 1-pfaffienne si et seulement le point col est intégrable.

On va donc analyser l'intégrabilité du col pour le feuilletage défini par (5.4).

\subsubsection{Intégrabilité du col}

Le problème est de savoir si le feuilletage défini par (5.2) admet une intégrale première analytique au voisinage du col et qui dépende aussi analytiquement de $\lambda$. En particulier on veut savoir si l'équation

$$
\ddot{\theta}+\sin \theta+2 \varepsilon \cos \theta \dot{\theta}=0
$$

où $2 \varepsilon=\frac{\beta}{\sqrt{\lambda}}$ est intégrable analytiquement au voisinage du col : $\theta=\pi, \dot{\theta}=0$. Pour $\beta=0$ c'est le cas car le système admet l'intégrale première globale : $I=\frac{1}{2} \dot{\theta}^{2}-\cos \theta$. Pour $\beta \neq 0$, on introduit : $x=\theta-\pi$ et $y=\dot{\theta}$ et on doit étudier le système :

$$
\begin{aligned}
& \dot{x}=y \\
& \dot{y}=x+2 \varepsilon y+f(x, y)
\end{aligned}
$$

où l'on a posé $f(x, y)=(\sin x-x)+2 \varepsilon y(\cos x-1)$.

Le spectre du linéarisé en 0 est $\left(\sigma_{1}, \sigma_{2}\right)$ où $\sigma_{1}=\varepsilon+\sqrt{\varepsilon^{2}+1}, \sigma_{2}=\varepsilon-\sqrt{\varepsilon^{2}+1}$. Lorsque $\lambda$ décrit $\mathbf{R}^{+}, \sigma_{1}, \sigma_{2}$ décrivent pour $\beta \neq 0$ un semi-intervalle dont une des extrémités est obtenue pour $\lambda \rightarrow+\infty$ et vaut $\sigma_{1}^{+}=1$ (resp. $\sigma_{2}^{-}=-1$ ). Pour $\lambda \gg \beta$ fixé la théorie classique de la linéarisation nous dit les choses suivantes :

- Cas a (cas non résonnant) : Si $\frac{\sigma_{1}}{\sigma_{2}}$ n'appartient pas à $\mathbf{Q}$ le système est linéarisable dans la classe $C^{\infty}$ et admet une intégrable première $C^{\infty}$.

- Cas b (cas résonnant) : $\mathrm{Si} \frac{\sigma_{1}}{\sigma_{2}} \in \mathbf{Q}$, le système admet une forme normale formelle qui est intégrable, et contenant une infinité de monômes résonnants. 
Par ailleurs, dans le cas non résonnant si le spectre vérifie des conditions diophantines alors le col est linéarisable dans la catégorie analytique et admet donc une intégrale première analytique. D'autre part l'ensemble des systèmes qui ne vérifient pas les conditions diophantines est partout dense. Donc comme dans notre cas le rapport $\frac{\sigma_{1}}{\sigma_{2}}$ décrit lorsque $\beta \neq 0$ une famille à un paramètre on peut conjecturer :

Conjecture 5.9. Il existe $\varepsilon \sim 0$ tel que le système (5.11) ne soit pas intégrable au voisinage du point col $(0,0)$.

Pour prouver cette conjecture, il faut montrer que pour certains $\varepsilon \sim 0$ et $\frac{\sigma_{1}(\varepsilon)}{\sigma_{2}(\varepsilon)}$ n'appartenant pas à $\mathbf{Q}$ la transformation linéarisante n'est pas convergente. Pour cela on travaille dans les coordonnées associées aux vecteurs propres du linéarisé :

$$
X=\frac{\sigma_{2} x-y}{\sigma_{2}-\sigma_{1}}, \quad Y=\frac{y-\sigma_{1} x}{\sigma_{2}-\sigma_{1}}
$$

où le système s'écrit :

$$
\begin{aligned}
& \dot{X}=\sigma_{1} X-\frac{f}{\sigma_{2}-\sigma_{1}} \\
& \dot{Y}=\sigma_{2} Y+\frac{f}{\sigma_{2}-\sigma_{1}}
\end{aligned}
$$

et $f(x, y)=(\sin x-x)+2 \varepsilon y(\cos x-1)=\left(-\frac{x^{3}}{3 !}+\frac{x^{5}}{5 !}+\ldots\right)+2 \varepsilon y\left(-\frac{x^{2}}{2 !}+\frac{x^{4}}{4 !}+\ldots\right)$ où $: x=X+Y, Y=\sigma_{1} X+\sigma_{2} Y$.

L'examen du second terme de $f$ semble indiquer que l'on ne peut pas compenser les petits dénominateurs, mais cela exige un calcul précis.

En utilisant la conjecture 5.9 et le théorème 5.8 de Mourtada-Moussu on peut donc conjecturer.

Conjecture 5.10. Pour $\beta \neq 0$, la sphère sous-riemannienne n'est pas sous-pfaffienne.

\subsubsection{Remarques sur l'évaluation de l'application premier retour}

Une remarque importante pour le calcul de l'application premier retour est la suivante. Pour $\lambda>>\beta$, la contrainte (5.5) devient $\dot{\theta} \sim 0$ et la séparatrice $\Sigma$ tend au sens $C^{1}$ vers la connection de cols du cas plat approximée en $\theta=-\pi$ par la direction propre $\dot{\theta}=0$ (voir figure 46). Donc comme dans le cas plat, la séparatrice $\Sigma$ ne correspond pas à une géodésique issue de 0 . On évite donc $($ si $\alpha=0)$ le phénomène de doublement de période, ce qui est important pour le calcul de bons développements asymptotiques.

Une autre remarque aussi importante est que l'on peut utiliser la formule de balance d'énergie $(E)$ pour faire des estimés pour l'application premier retour. Enfin le calcul des développements asymptotiques de la sphère peut-être fait en utilisant les techniques de [32]. Ces techniques reposent sur l'existence d'une intégrale première formelle au voisinage d'un col.

\section{Conclusion}

Dans cet article on a introduit un outil important pour étudier la géométrie SR en présence de géodésiques anormales minimisantes, c'est le feuilletage :

$$
\theta^{\prime \prime}+\lambda \sin \theta+\alpha^{2} \sin \theta \cos \theta-\alpha \beta \sin ^{2} \theta+\beta \cos \theta \theta^{\prime}=0
$$

et la contrainte induite par la condition $y=0$ :

$$
\theta^{\prime}=(\alpha \cos \theta-\beta \sin \theta)
$$

où l'on suppose $\lambda>>\beta, \alpha$.

On a obtenu une caractérisation géométrique très fine du rôle des paramètres $\alpha, \beta, \gamma$ dans la forme normale. On a montré par des considérations géométriques un résultat d'optimalité très précis : pour les trois déformations 
à un paramètre étudiées, la trace de la sphère avec le plan $y=0$ est contenue dans l'image de l'application premier et second retour. On peut conjecturer que c'est vrai en général. Enfin on a prouvé que le modèle plat présente certaines propriétés de stabillité et est donc un bon modèle pour étudier le cas générique.

Pour clore l'étude du cas Martinet les trois points suivants doivent être examinés en détails :

- Calcul de la transcendance exp-log précise de la sphère de petit rayon dans le cas intégrable.

- Évaluation de la trace de la sphère de petit rayon avec le plan de Martinet dans le cas non intégrable.

- Évaluation du lieu conjugé et du lieu de coupure.

Ces trois études sont fondamentales et aucune n'est triviale. Le premier calcul est l'objet de l'article [9]. Le second calcul est l'objet de l'article [10] en cours de rédaction où l'on étudie aussi la généralisation de nos résultats en dimension quelconque. Enfin le troisième point est cours d'étude, en particulier on analyse le lieu de coupure et le lieu conjugué dans le cas intégrable.

\section{Appendice : ÉlÉMents de la théORIE DES FOnCTIONS ELLIPTIQUES}

\subsection{Introduction}

L'objectif de ce chapitre est d'introduire les fonctions elliptiques et de présenter leurs principales propriétés. Cette introduction est motivée par notre étude de géométrie sous-riemannienne. Elle est donc très incomplète, en particulier on se limite au domaine réel. Le lecteur intéressé par une étude plus complète du sujet peut consulter les ouvrages de référence de Halphen et Tannery-Molk [16,35]. Pour notre part on a essentiellement utilisé le livre de Lawden [21] qui fait une présentation plus moderne du sujet et qui est par ailleurs un bon glossaire de formules. L'exemple de la théorie de l'élasticité provient de l'excellent livre de Stoker [33].

\subsection{Les fonctions elliptiques sn, cn et dn de Jacobi}

Definition 7.1. Soit $k$ un paramètre réel compris strictement entre 0 et 1 et $\eta$ un réel, $0 \leq \eta \leq 1$. La fonction réciproque de la fonction cn est par définition

$$
\operatorname{cn}^{-1}(\eta, k)=\int_{\eta}^{1} \frac{d t}{\sqrt{\left(1-t^{2}\right)\left(k^{\prime 2}+k^{2} t^{2}\right)}}
$$

où $k$ est le module et $0<k^{\prime}<1$ est le complémentaire du module défini par : $k^{2}+k^{\prime 2}=1$.

Il résulte directement de notre définition le résultat suivant.

\subsubsection{Propriété}

La fonction $\eta(t)=\operatorname{cn}(t, k)$ est solution de l'équation différentielle :

$$
\left(\frac{d \eta}{d t}\right)^{2}=\left(1-\eta^{2}\right)\left(k^{\prime 2}+k^{2} \eta^{2}\right)
$$

C'est une fonction périodique dont la période est $4 K(k)$ où $K(k)$ est l'intégrale complète de première espèce :

$$
K(k)=\int_{0}^{1} \frac{d \eta}{\sqrt{\left(1-\eta^{2}\right)\left(k^{\prime 2}+k^{2} \eta^{2}\right)}}=\int_{0}^{\frac{\pi}{2}} \frac{d \theta}{\sqrt{1-k^{2} \sin ^{2} \theta}} .
$$

De la même façon on définit les fonctions sn et dn.

Definition 7.2. Soit $0<k<1$ et $0 \leq \eta \leq 1$, on pose :

$$
\operatorname{sn}^{-1}(\eta, k)=\int_{0}^{\eta} \frac{d t}{\sqrt{\left(1-t^{2}\right)\left(1-k^{2} t^{2}\right)}} \cdot
$$


La fonction $\eta(t)=\operatorname{sn}(t, k)$ est une fonction périodique de période $4 K(k)$ solution de l'équation :

$$
\left(\frac{d \eta}{d t}\right)^{2}=\left(1-\eta^{2}\right)\left(1-k^{2} \eta^{2}\right)
$$

Definition 7.3. Soit $0<b<1$ et $b \leq \eta \geq 1$, la fonction $\eta(t)=\operatorname{dn}(t, k)$ où $k=\sqrt{1-b^{2}}$ est par définition :

$$
\operatorname{dn}^{-1}(\eta, k)=\int_{\eta}^{1} \frac{d t}{\sqrt{\left(1-t^{2}\right)\left(t^{2}-b^{2}\right)}}
$$

C'est une fonction périodique de période $2 K(k)$ solution de l'équation :

$$
\left(\frac{d \eta}{d t}\right)^{2}=\left(1-\eta^{2}\right)\left(\eta^{2}-b^{2}\right)
$$

\subsubsection{Propriété}

On a les relations suivantes :

$$
\begin{aligned}
\operatorname{sn}^{2} t+\mathrm{cn}^{2} t & =1 \\
\mathrm{dn}^{2} t+k^{2} \operatorname{sn}^{2} t & =1 \\
\operatorname{dn}^{2} t-k^{2} \mathrm{cn}^{2} t & =k^{\prime 2}
\end{aligned}
$$

Remarque 7.4. Ces fonctions représentent la transition entre les fonctions trigonométriques et les fonctions hyperboliques et l'on a:

- lorsque $k \rightarrow 0$,

$$
\operatorname{sn}(t, k) \rightarrow \sin t, \quad \operatorname{cn}(t, k) \rightarrow \cos t, \quad \operatorname{dn}(t, k) \rightarrow 1
$$

- lorsque $k \rightarrow 1$,

$$
\operatorname{sn}(t, k) \rightarrow \tanh t, \quad \operatorname{cn}(t, k), \operatorname{dn}(t, k) \rightarrow \operatorname{sech} t .
$$

\subsection{Intégrales elliptiques de première et seconde espèce - Fonction $E$}

Definition 7.5. On appelle intégrale elliptique de première espèce une intégrale de la forme :

$$
\int \frac{d t}{\sqrt{\left(A_{1} t^{2}+B_{1}\right)\left(A_{2} t^{2}+B_{2}\right)}}, \quad A_{1}, A_{2}, B_{1}, B_{2} \in \mathbf{R}
$$

et intégrale elliptique de deuxième espèce une intégrale de la forme :

$$
\int \frac{t^{2} d t}{\sqrt{\left(A_{1} t^{2}+B_{1}\right)\left(A_{2} t^{2}+B_{2}\right)}}, \quad A_{1}, A_{2}, B_{1}, B_{2} \in \mathbf{R} .
$$

Definition 7.6. La fonction Epsilon de Jacobi est définie par :

$$
E(t, k)=\int_{0}^{t} \operatorname{dn}^{2}(v, k) d v
$$


et l'intégrale elliptique complète de deuxième espèce est donnée par :

$$
E(k)=E(K, k)=\int_{0}^{K} \operatorname{dn}^{2}(v, k) d v .
$$

\subsubsection{Propriété}

En posant $u=\operatorname{sn} v$ et $\tau=\operatorname{sn} t$ on obtient l'expression :

$$
E(t, k)=\int_{0}^{\tau} \sqrt{\frac{1-k^{2} u^{2}}{1-u^{2}}} d u
$$

De façon plus générale on a le résultat suivant.

Proposition 7.7. Toute intégrale de première et de seconde espèce se ramène à l'évaluation des fonctions de Jacobi sn, cn, dn et $E$ et en particulier de deux intégrales fondamentales :

$$
\begin{aligned}
& K(\phi, k)=\int_{0}^{\phi} \frac{d \theta}{\sqrt{1-k^{2} \sin ^{2} \theta}} \\
& E(\phi, k)=\int_{0}^{\phi}\left(\sqrt{1-k^{2} \sin ^{2} \theta}\right) d \theta
\end{aligned}
$$

où $\phi$ est un angle compris entre 0 et $\frac{\pi}{2}$.

\subsubsection{Propriété}

La fonction $E$ n'est pas périodique mais vérifie l'égalité

$$
E(t+2 K)=E(t)+2 E(K)
$$

et l'on peut extraire de $E$ une fonction périodique de période $2 K$ en introduisant la fonction Zeta de Jacobi :

$$
Z(t)=E(t)-\frac{E(K) t}{K}
$$

Les comportements limites sont les suivants :

$$
\lim _{k \rightarrow 0} E(t, k)=t, \quad \lim _{k \rightarrow 1} E(t, k)=\tanh t
$$

\subsection{Formules intégrales}

On a les relations suivantes :

$$
\begin{aligned}
\int \operatorname{cn} t d t & =\frac{1}{k} \arcsin (k \operatorname{sn} t) \\
\int \operatorname{dn} t d t & =\arcsin (\operatorname{sn} t) \\
k^{2} \int \mathrm{sn}^{2} t d t & =t-\int \mathrm{dn}^{2} t d t \\
k^{2} \int \mathrm{cn}^{2} t d t & =\int \mathrm{dn}^{2} t d t-k^{\prime 2} t .
\end{aligned}
$$


En posant :

$$
J_{m}=\int \mathrm{cn}^{m} t d t, \quad K_{m}=\int \mathrm{dn}^{m} t d t,
$$

on calcule $J_{m}$ et $K_{m}$ par récurrence avec les formules :

$$
\begin{aligned}
(m+1) k^{2} J_{m+2} & =m\left(2 k^{2}-1\right) J_{m}+(m-1) k^{\prime 2} J_{m-2}+\mathrm{cn}^{m-1} t \operatorname{sn} t \operatorname{dn} t, \\
(m+1) K_{m+2} & =m\left(2-k^{2}\right) K_{m}-(m-1) k^{\prime 2} K_{m-2}+k^{2} \operatorname{dn}^{m-1} t \operatorname{sn} t \operatorname{cn} t .
\end{aligned}
$$

\subsection{Intégrales complètes et leurs propriétés}

Definition 7.8. L'intégrale complète de première espèce est :

$$
K(k)=\int_{0}^{\frac{\pi}{2}} \frac{d \theta}{\sqrt{1-k^{2} \sin ^{2} \theta}}
$$

et l'intégrale complète de seconde espèce est :

$$
E(k)=\int_{0}^{\frac{\pi}{2}} \sqrt{1-k^{2} \sin ^{2} \theta} d \theta .
$$

Leurs complémentaires sont définis respectivement par :

$$
K^{\prime}(k)=K\left(k^{\prime}\right), \quad E^{\prime}(k)=E(k) .
$$

Dans notre étude il est crucial de connaître les développements asymptotiques de $E$ et $K$ lorsque $k \rightarrow 0$ et $k \rightarrow 1$. Les coefficients se calculent par récurrence en utilisant les définitions intégrales ou la propriété suivante exprimant le fait que $E$ et $K$ sont solutions d'équations différentielles hypergéométriques.

Lemme 7.9. On a les relations suivantes :

$$
\begin{aligned}
& \frac{d K}{d k}=\frac{1}{k k^{\prime 2}}\left(E-k^{\prime 2} K\right) \\
& \frac{d E}{d k}=\frac{1}{k}(E-K)
\end{aligned}
$$

et $w=A K^{\prime}+B K$ est la solution générale de l'équation :

$$
k\left(1-k^{2}\right) \frac{d^{2} w}{d k^{2}}+\left(1-3 k^{2}\right) \frac{d w}{d k}-k w=0
$$

et $E$ est solution de l'équation :

$$
k\left(1-k^{2}\right) \frac{d^{2} w}{d k^{2}}+\left(1-k^{2}\right) \frac{d w}{d k}+k w=0 .
$$

On en déduit les développements asymptotiques convergents suivants. 
Lemme 7.10. Lorsque $k \rightarrow 0$, on $a:$

$$
\begin{aligned}
& K(k)=\frac{\pi}{2}\left[1+\left(\frac{1}{2}\right)^{2} k^{2}+\left(\frac{1}{2} \frac{3}{4}\right)^{2} k^{4}+\ldots\right] \\
& E(k)=\frac{\pi}{2}\left[1-\left(\frac{1}{2}\right)^{2} k^{2}-\frac{1}{3}\left(\frac{1}{2} \frac{3}{4}\right)^{2} k^{4}-\ldots\right] .
\end{aligned}
$$

De même lorsque $k \rightarrow 1$, on a le résultat suivant.

Lemme 7.11. Lorsque $k \rightarrow 1$, on $a:$

$$
\begin{aligned}
& E(k)=u_{1}\left(k^{\prime}\right) \log \frac{4}{k^{\prime}}+u_{2}\left(k^{\prime}\right) \\
& K(k)=u_{3}\left(k^{\prime}\right) \log \frac{4}{k^{\prime}}+u_{4}\left(k^{\prime}\right)
\end{aligned}
$$

où $k^{\prime}=\sqrt{1-k^{2}}$ et les $u_{i}$ sont des fonctions analytiques de $k^{\prime}$ en 0 dont les développements sont:

$$
\begin{aligned}
& u_{1}\left(k^{\prime}\right)=\frac{k^{\prime 2}}{2}+o\left(k^{\prime 3}\right), \quad u_{2}\left(k^{\prime}\right)=1-\frac{k^{\prime 2}}{4}+o\left(k^{\prime 3}\right) \\
& u_{3}\left(k^{\prime}\right)=1+\frac{k^{\prime 2}}{4}+o\left(k^{\prime 3}\right), \quad u_{4}\left(k^{\prime}\right)=-\frac{k^{\prime 2}}{4}+o\left(k^{\prime 3}\right) .
\end{aligned}
$$

Remarque 7.12. Les développements complets sont donnés dans [12] pp. 133-134. La propriété fondamentale des intégrales complètes à retenir pour notre étude est la suivante.

\subsubsection{Propriété}

Lorsque $k \rightarrow 0$, les intégrales complètes $K(k)$ et $E(k)$ sont des fonctions analytiques et lorsque $k \rightarrow 1$, elles admettent des singularités logarithmiques. En particulier : $K(k) \sim \log \frac{4}{k^{\prime}} \operatorname{lorsque} k^{\prime} \rightarrow 0$.

\subsection{Les modèles physiques}

Les fonctions elliptiques apparaissent de façon naturelle dans la modélisation de systèmes physiques, on peut d'ailleurs les définir comme des solutions de problèmes physiques. On a choisi de présenter deux exemples liés de façon fondamentale à notre étude. L'un est classique c'est l'exemple du pendule simple. L'autre est moins connu et provient de la théorie de l'élasticité. C'est un modèle physique qui représente le comportement des géodésiques dans le cas Martinet intégrable générique et qui donne une explication à la rigidité en géométrie SR.

\subsection{Le pendule simple}

Soit un pendule simple de longueur $l, \theta$ l'angle par rapport à la verticale. Son évolution est donnée par l'équation de conservation d'énergie :

$$
\dot{\theta}^{2}-2 \omega^{2} \cos \theta=C
$$

où $C$ est une constante et $\omega=\sqrt{\frac{g}{l}}$.

Pour calculer les solutions il faut distinguer deux types de trajectoires, celles qui correspondent à un pendule oscillant et celles qui correspondent à un pendule en rotation. Cela genère deux types bien distincts de trajectoires que l'on distingue aisément en considérant les cas où $\dot{\theta}$ s'annule ou non. 
7.7.1. Le pendule oscillant

En notant $\alpha$ l'amplitude angulaire de l'oscillation on a $\dot{\theta}=0$ quand $\theta=\alpha$ et l'on écrit :

$$
\dot{\theta}^{2}=2 \omega^{2}(\cos \theta-\cos \alpha)=4 \omega^{2}\left(\sin ^{2} \frac{\alpha}{2}-\sin ^{2} \frac{\theta}{2}\right) .
$$

En intégrant il vient :

$$
\omega t=\frac{1}{2} \int_{0}^{\theta} \frac{d \theta}{\sqrt{\sin ^{2} \frac{\alpha}{2}-\sin ^{2} \frac{\theta}{2}}} .
$$

En faisant le changement de variable :

$$
\sin \frac{\theta}{2}=\sin \frac{\alpha}{2} \sin \varphi
$$

il vient

$$
\omega t=\int_{0}^{\varphi} \frac{d \varphi}{\sqrt{1-\sin ^{2} \frac{\alpha}{2} \sin ^{2} \varphi}}=\operatorname{sn}^{-1}\left(\sin \varphi, \sin \frac{\alpha}{2}\right)
$$

Soit donc:

$$
\sin \frac{\theta}{2}=\sin \frac{\alpha}{2} \operatorname{sn}(\omega t, k)
$$

où $k=\sin \frac{\alpha}{2}$. La période des oscillations est $T=\frac{4 K}{\omega}$. Par ailleurs on en déduit que la vitesse angulaire vérifie

$$
\dot{\theta}=2 \omega \sin \frac{\alpha}{2} \operatorname{cn} \omega t
$$

et c'est donc une représentation physique de la fonction $\mathrm{cn}$.

\subsubsection{Le pendule de révolution}

Si la vitesse initiale $V$ du pendule est assez grande, celui-ci exécute alors des cercles complets. L'équation de conservation d'énergie s'écrit :

$$
\frac{1}{2} m v^{2}-m g l \cos \theta=\frac{1}{2} m V^{2}-m g l
$$

soit :

$$
\dot{\theta}^{2}=2 \omega^{2}\left(\frac{V^{2}}{2 g l}-1+\cos \theta\right)
$$

et pour $\theta=\pi$, on impose $\dot{\theta}^{2}>0$ soit $V^{2}>4 g l$. En posant :

$$
\phi=\frac{\theta}{2} \text { et } k^{2}=\frac{4 g l}{V^{2}}, 0<k<1,
$$

l'équation s'écrit :

$$
\dot{\phi}^{2}=\frac{\omega^{2}}{k^{2}}\left(1-k^{2} \sin ^{2} \phi\right)
$$



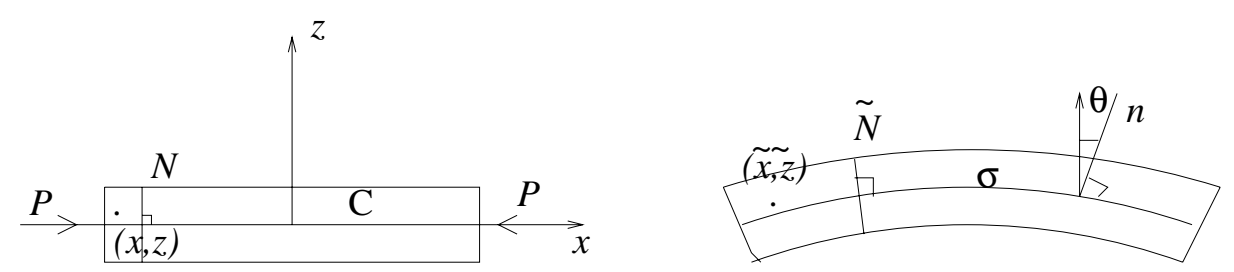

FiguRe 48

et en intégrant il vient :

$$
\sin \phi=\sin \frac{\theta}{2}=\operatorname{sn}\left(\frac{\omega t}{k}, k\right)
$$

L'angle $\theta$ varie donc de façon périodique et la période est ici :

$$
T=\frac{2 k K}{\omega}=\frac{4 l K}{V} .
$$

En dérivant il vient :

$$
\dot{\theta}=\frac{2 \omega}{k} \operatorname{dn}\left(\frac{\omega t}{k}, k\right)
$$

et c'est donc une représentation physique de la fonction $\mathrm{dn}$.

\subsubsection{Le cas critique}

C'est la situation où $\alpha=\pi$. La période tend alors vers l'infini et les fonctions cn et dn dégénèrent en des fonctions hyperboliques. Ce cas représente la transition entre le cas oscillant et le cas de révolution.

\subsubsection{Le pendule linéarisé}

Pour des oscillations de faible amplitude $\alpha, k=\sin \frac{\alpha}{2}$ tend vers 0 et $K(k)$ tend vers $\frac{\pi}{2}$. On peut alors linéariser les équations car les fonctions de Jacobi dégénèrent en des fonctions trigonométriques.

\subsubsection{Conclusion}

Le pendule est la modélisation physique des fonctions elliptiques de premières espèce et par ailleurs il illustre physiquement le rôle des fonctions elliptiques comme une transition entre les fonctions trigonométriques et les fonctions hyperboliques.

Dans notre problème de géométrie SR c'est le modèle physique fondamental.

\subsection{La barre élastique (d'après [33])}

\subsubsection{Le modèle physique}

On considère le problème de la déformation d'une barre élastique de longueur $2 L$ au repos, planaire et homogène (Fig. 48).

On note $C$ la ligne centrale au repos, paramétrisée par $x \in[-L,+L]$ et qui se déforme en $\sigma: x \mapsto(X(x), Z(x))$. On fait l'hypothèse qu'un plan $N$ normal à cette ligne reste normal après déformation. Si l'image d'un point 
$(x, z)$ est notée $(\tilde{x}, \tilde{z})$ où le référentiel est indiqué sur la figure on a alors les relations :

$$
\begin{aligned}
& \tilde{x}=X(x)+\eta(x, z) \xi(x) \\
& \tilde{z}=Z(x)+\eta(x, z) \psi(x)
\end{aligned}
$$

où $(\xi, \psi)$ représentent les composantes du vecteur $n$ normal à la ligne centrale : $x \mapsto \sigma(x)$. La fonction $\eta$ représente la déformation des plans normaux. On note $\theta$ l'angle de la normale $n$ avec la verticale.

On fait l'hypothèse que la déformation est due à l'application d'une force de compression $\vec{P}$ appliquée aux extrémités dans la direction $O x$ et on note $P$ son module.

On note $\tilde{u}, \tilde{w}$ les composantes respectives de la déformation :

$$
\tilde{u}=\tilde{x}-x \text { et } \tilde{w}=\tilde{z}-z
$$

et $u, v$ les déformations de la ligne centrale :

$$
u=X(x)-x, w=Z(x)
$$

et l'on peut imposer différentes hypothèses sur les déformations aux extrémités. On fera l'hypothèse suivante : (H1): $w( \pm L)=0$ qui signifie géométriquement que les extrémités de la ligne centrale restent après déformation sur l'axe $O x$.

\subsubsection{Les équations}

Pour modéliser la position prise par la barre on utilise l'approche variationnelle. On procède ainsi. La longueur d'arc de la ligne centrale : $x \mapsto \sigma(x)$ est :

$$
d s=\left(\sqrt{X_{x}^{2}+Z_{x}^{2}}\right) d x=\left(\sqrt{\left(1+u_{x}\right)^{2}+w_{x}^{2}}\right) d x
$$

et l'on écrit :

$$
d s=(1+\varepsilon(x)) d x .
$$

On a aussi

$$
\begin{aligned}
& \xi=-\sin \theta=-\frac{w_{x}}{1+\varepsilon} \\
& \psi=\cos \theta=\frac{1+u_{x}}{1+\varepsilon}
\end{aligned}
$$

et le paramètre géométrique important est la courbure de la courbe $\sigma$ définie par l'équation :

$$
\kappa=\theta_{s}=\frac{\theta_{x}}{1+\varepsilon}
$$

Pour calculer la position prise par la barre on doit faire une modélisation de la déformation d'un plan normal, c'est-à-dire donner la fonction $\eta$ et l'on supposera ici que : (H2): $\eta(x, z)=z$ mais d'autres hypothèses sont possibles conduisant à la même équation pour la ligne centrale. 
Le tenseur de déformation $N=\left(\begin{array}{ll}\varepsilon_{11} & \varepsilon_{12} \\ \varepsilon_{12} & \varepsilon_{22}\end{array}\right)$ est défini dans la théorie par la relation :

$$
(1+N)^{2}=\left(1+{ }^{t} Q\right)(1+Q)
$$

où $(1+Q)$ est la matrice de déformation :

$$
(1+Q)=\left(\begin{array}{cc}
1+\tilde{u}_{x} & \tilde{u}_{z} \\
\tilde{w}_{x} & 1+\tilde{w}_{z}
\end{array}\right)
$$

et en calculant avec $\eta=z$ il vient :

$$
N=\left(\begin{array}{cc}
\varepsilon-(1+\varepsilon) \kappa z & 0 \\
0 & 0
\end{array}\right)
$$

Pour obtenir la position de la barre en utilisant le principe variationnel on doit modéliser l'énergie interne et on utilise pour cela la loi de Hooke où l'énergie d'un élément de volume est prise comme :

$$
d V=\frac{E b}{2} \varepsilon_{11}^{2} d x d z
$$

où $E$ et $b$ sont des constantes physiques et l'énergie interne est donc :

$$
V=\frac{E b}{2} \int_{-h}^{+h} \int_{-L}^{+L}[\varepsilon-(1+\varepsilon) \kappa z]^{2} d x d z
$$

où $2 h$ est la largeur. On obtient :

$$
V=\frac{E}{2} \int_{-L}^{+L}\left(A \varepsilon^{2}(x)+I \theta_{x}^{2}(x)\right) d x
$$

où l'on a posé :

$$
I=b \int_{-h}^{+h} z^{2} d z \quad \text { et } \quad A=2 b h .
$$

L'énergie externe est l'énergie potentielle due à la compression :

$$
P(u(L))-u(-L))
$$

et l'énergie totale est donc :

$$
\mathbf{w}=\int_{-L}^{+L}\left(\frac{E A \varepsilon^{2}}{2}+\frac{E I \theta_{x}^{2}}{2}\right) d x+P(u(L)-u(-L)) .
$$

Par ailleurs, par symétrie $u(0)=0$ et on peut écrire :

$$
u(x)=\int_{0}^{x} u_{x} d x=\int_{0}^{x}[(1+\varepsilon) \cos \theta-1] d x .
$$

Soit donc la forme intégrale :

$$
\mathbf{w}=\int_{-L}^{+L}\left\{\left(\frac{E A \varepsilon^{2}}{2}+\frac{E I \theta_{x}^{2}}{2}\right)+P[(1+\varepsilon) \cos \theta-1]\right\} d x .
$$


Le déplacement vertical est donné par :

$$
w_{x}=(1+\varepsilon) \sin \theta
$$

où l'on impose les conditions limites :

$$
w( \pm L)=0
$$

On obtient donc que la position de la barre est la solution du problème variationnel : Minw avec les conditions limites $w( \pm L)=0$.

Pour trouver la solution de ce problème variationnel on utilise la théorie du contrôle et le principe du maximum. On a donc le système $\Sigma$ :

$$
\begin{aligned}
\dot{u}(x) & =(1+\varepsilon) \cos \theta-1 \\
\dot{w}(x) & =(1+\varepsilon) \sin \theta \\
\dot{\theta} & =v
\end{aligned}
$$

où les contrôles sont la déformation $\varepsilon(x)$ et $v=\theta_{x}$, et le coût à minimiser est :

$$
\mathbf{w}=\int_{-L}^{+L}\left\{\left(\frac{E A \varepsilon^{2}}{2}+\frac{E I \theta_{x}^{2}}{2}\right)+P[(1+\varepsilon) \cos \theta-1]\right\} d x .
$$

Introduisons le coût comme composante de l'état en adjoignant à (7.1) l'équation :

$$
\dot{\mathbf{w}}=\frac{E A \varepsilon^{2}}{2}+\frac{E I \theta_{x}^{2}}{2}+P[(1+\varepsilon) \cos \theta-1]
$$

En notant $p=\left(p_{u}, p_{w}, p_{\theta}, p_{\mathbf{w}}\right)$ le vecteur adjoint et en tenant compte du fait que les extrémités $u$ et $\theta$ sont libres, le vecteur vérifie donc les conditions de transversalité suivantes :

$$
p_{u}( \pm L)=p_{\theta}( \pm L)=0
$$

et

$$
\dot{p}_{\theta}=-p_{w}(1+\varepsilon) \cos \theta+p_{\mathbf{w}} P(1+\varepsilon) \sin \theta
$$

car $p_{u}$ est constant et $p_{u}( \pm L)=0$, donc $p_{u}=0$. Le pseudo-hamiltonien s'écrit :

$$
H=p_{w}(1+v) \sin \theta+p_{\theta} v+p_{\mathbf{w}}\left[\frac{E A \varepsilon^{2}}{2}+\frac{E I \theta_{x}^{2}}{2}+P[(1+\varepsilon) \cos \theta-1]\right]
$$

et $p_{\mathbf{w}} \leq 0$. Dans le cas normal on peut choisir $p_{\mathbf{w}}=-1$ et le contrôle est calculé en utilisant :

$$
\begin{aligned}
& \frac{\partial H}{\partial v}=p_{w} \sin \theta+p_{\theta}+p_{\mathbf{w}} E I v=0 \\
& \frac{\partial H}{\partial \varepsilon}=p_{\mathbf{w}}[E A \varepsilon+P \cos \theta]=0 .
\end{aligned}
$$

Par ailleurs en intégrant (7.4) il vient :

$$
p_{\theta}(L)-p_{\theta}(-L)=-p_{w} \int_{-L}^{+L}(1+\varepsilon) \cos \theta d x+p_{\mathbf{w}} P \int_{-L}^{+L}(1+\varepsilon) \sin \theta d x=0
$$

et en utilisant :

$$
w( \pm L)=0, \int_{-L}^{+L}(1+\varepsilon) \sin \theta d x=w(L)-w(-L)=0
$$


il vient :

$$
-p_{w} \int_{-L}^{+L}(1+\varepsilon) \cos \theta d x=0
$$

On a donc soit $p_{w}=0$ soit $\int_{-L}^{+L}(1+\varepsilon) \cos \theta d x=0$. La solution physique est associée à $p_{w}=0$ et l'on a alors :

$$
\theta_{x}=v=\frac{p_{\theta}}{E I} \text { et } \varepsilon=-\frac{P \cos \theta}{E A}
$$

et de la seconde équation on tire

$$
\varepsilon=\varepsilon_{0} \cos \theta, \quad \varepsilon_{0}=-\frac{P}{E A} .
$$

En dérivant la première équation on obtient l'équation fondamentale :

$$
\theta_{x x}+\lambda^{2}\left(1+\varepsilon_{0} \cos \theta\right) \sin \theta=0
$$

où $\lambda^{2}=\frac{P}{E I}=-\frac{A \varepsilon_{0}}{I}$. En intégrant entre $-L$ et $+L$ il vient :

$$
\int_{-L}^{+L} \theta_{x x} d x=-\frac{P}{E I} \int_{-L}^{+L}(1+\varepsilon) \sin \theta d x
$$

où le second membre est 0 car $w( \pm L)=0$. On obtient donc :

$$
\theta_{x}(L)-\theta_{x}(-L)=0
$$

Par ailleurs du fait de la symétrie des solutions de (7.7) par rapport à 0 , on a :

$$
\theta_{x}(L)=-\theta_{x}(-L)=0
$$

et l'on obtient : $\theta_{x}( \pm L)=0$. Les extrémités de la barre sont des points d'inflexion. Par ailleurs une autre conséquence de cette symétrie est $\theta(0)=0$. Cela détermine donc la solution de (7.7). Pour intégrer on utilise

$$
\theta_{x}^{2}=2 \lambda^{2}\left[\cos \theta-\cos \theta_{1}+\frac{\varepsilon_{0}}{2}\left(\cos ^{2} \theta-\cos ^{2} \theta_{1}\right)\right]
$$

où l'on a posé : $\theta_{1}=\theta(-L)$. On introduit :

$$
k=\frac{\sin \theta_{1}}{2}, \quad k \sin \phi=\sin \frac{\theta}{2}
$$

et l'on obtient :

$$
x=-\frac{1}{\lambda} \int_{0}^{\phi} \frac{d \varphi}{\sqrt{\left.1-k^{2} \sin ^{2} \varphi \sqrt{1+\varepsilon_{0}\left(1-k^{2}\left(1+\sin ^{2} \varphi\right)\right.}\right)}}=-\frac{1}{\lambda} F\left(\varepsilon_{0}, k, \phi\right) .
$$

Pour $x=-L$, on a $\phi=\frac{\pi}{2}$ et cela impose donc la condition :

$$
L=\frac{1}{\lambda} F\left(\varepsilon_{0}, k, \frac{\pi}{2}\right)
$$


ce qui entraine la contrainte :

$$
P=E I \lambda^{2}=\frac{E I}{L^{2}}\left(F\left(\varepsilon_{0}, k, \frac{\pi}{2}\right)\right)^{2} .
$$

Cette relation a une signification physique importante. Elle signifie que la déformation a lieu uniquement pour une compression assez forte, c'est le phénomène dit de flambage. On peut l'estimer en prenant par exemple $\varepsilon_{0} \sim 0, F\left(\varepsilon_{0}, k, \frac{\pi}{2}\right) \sim K(k)$ et la valeur minimale est obtenue pour $k=0$ et vaut $K(0)=\frac{\pi}{2}$. On obtient donc :

$$
P>P_{0}=\frac{\pi^{2} E I}{4 L^{2}}
$$

\subsubsection{Le lien avec l'élastique d'Euler}

Considérons l'équation fondamentale (7.7) :

$$
E I \theta_{x x}+P\left(1+\varepsilon_{0} \cos \theta\right) \sin \theta=0
$$

où $\varepsilon(x)=\varepsilon_{0} \cos \theta$ et $w_{x}=(1+\varepsilon) \sin \theta$. En intégrant entre $-L$ et $x$ avec les condition limites : $\theta_{x}(-L)=$ $w(-L)=0$ il vient :

$$
E I \theta_{x}=-P w .
$$

En utilisant alors la relation : $\theta_{x}=(1+\varepsilon) \kappa$, on obtient :

$$
-(1+\varepsilon) E I \kappa=P w .
$$

C'est l'équation classique de l'élastique d'Euler si l'on suppose maintenant $\varepsilon$ constant. Avec $\kappa=\theta_{s}$ et en dérivant par rapport à $s$ on obtient en effet :

$$
\theta_{s s}+\frac{P}{(1+\varepsilon) E I} \sin \theta=0
$$

L'étude a été faite par Euler et est bien donnée dans [28]. La forme de l'élastique inflexionel est précisement décrite par la projection d'une géodésique normale de la géométrie SR dans le cas Martinet plat, dans le plan $(x, y)$.

\subsubsection{Trajectoires résiduelles}

Le système $\Sigma$ s'écrit :

$$
\dot{x}=F_{0}(x)+v(t) F_{1}(x)+\varepsilon(t) F_{2}(x)
$$

où $x=(u, v, w)$ et $F_{0}, F_{1}, F_{2}$ sont les champs de vecteurs :

$$
\begin{aligned}
& F_{0}=(\cos \theta-1) \frac{\partial}{\partial u}+\sin \theta \frac{\partial}{\partial w} \\
& F_{1}=\frac{\partial}{\partial \theta}, \quad F_{2}=\cos \theta \frac{\partial}{\partial u}+\sin \theta \frac{\partial}{\partial w} .
\end{aligned}
$$

En calculant il vient :

$$
\left[F_{1}, F_{0}\right]=\left[F_{1}, F_{2}\right]=-\sin \theta \frac{\partial}{\partial u}+\cos \theta \frac{\partial}{\partial w}
$$

et $\left[F_{0}, F_{2}\right]=0$. Les contrôles singuliers du système affine $(\Sigma)$ vérifient les équations :

$$
\operatorname{det}\left(F_{1}, F_{2},\left[F_{1}, F_{0}\right]+\varepsilon\left[F_{1}, F_{2}\right]\right)=0
$$

et

$$
\operatorname{det}\left(F_{1}, F_{2},\left[F_{2}, F_{0}\right]+v\left[F_{2}, F_{1}\right]\right)=0 .
$$


On obtient donc $v=0$ et $\varepsilon=-1$. Ce cas est exclu physiquement car si $\varepsilon=-1$, cela signifie que $d s=0$ ce qui est absurde. En particulier on observe que la forme de la barre avant sa déformation qui vérifie $\theta=0$ et $\varepsilon=0$ n'est pas une trajectoire singulière.

Une autre famille de solutions correspondent à $\int_{-L}^{+L}(1+\varepsilon(x)) \cos \theta(x) d x=0$. Là encore $\theta(x)=0$ et $\varepsilon(x)=0$ n'est pas solution. Ces trajectoires vérifient la contrainte $u(L)-u(-L)+2 L=0$.

\subsubsection{Conclusion}

On a donc trouvé un modèle physique qui correspond au feuilletage $(\mathcal{F})$ dans le cas $\beta=0$ et qui décrit le comportement des géodésiques normales telles que $\theta^{\prime}$ s'annule. Ce modèle donne une explication physique à la rigidité géodésique. C'est un phénomène de flambage : pour qu'aux extrémités on ait des points d'inflexions il faut que la compression $P$ soit assez forte.

\section{REFERENCES}

[1] A. Agrachev, B. Bonnard, M. Chyba and I. Kupka Sub-Riemannian sphere in Martinet flat case. ESAIM:COCV 2 (1997) $377-448$.

[2] A. Agrachev, C. El Alaoui and J.P. Gauthier, Sub-Riemannian metrics on $\mathbf{R}^{3}$. Geometric Control and Non-holonomic Problems in Mechanics, Conference Proceedings Series, Canad. Math. Soc. (to appear).

[3] A.A. Agrachev and R.V. Gamkrelidze, Exponentional representations of flows and chronological calculus. Math. USSR Sb. 35 (1979) 727-785.

[4] A.A. Agrachev and A.V. Sarychev, Strong minimality of abnormal geodesics for 2- distributions. J. Dynamical and Control Systems 1 (1995) 139-176.

[5] A.A. Agrachev and A.V. Sarychev, Abnormal geodesics in SR-geometry subanalycity. Preprint (1997).

[6] A.A. Andronov, A.A. de Vitt and S.E. Khaikin, Theory of oscillations, Dover, New-York (1966).

[7] B. Bonnard, M. Chyba and I. Kupka, Non-integrable geodesics in SR-Martinet geometry, Proceedings AMS conference, Boulder (1997).

[8] B. Bonnard, M. Chyba and E. Trélat, Sub-Riemannian geometry: one parameter deformation of the Martinet flat case. J. Dynamical and Control Systems 4 (1998) 59-76.

[9] B. Bonnard, G. Launey and E. Trélat, The transcendence we need to compute the Sphere and the Wave Front in Martinet SR-Geometry. to appear in Proc. of Steklov Institute.

[10] B. Bonnard and E. Trélat, The role of abnormal minimizers in SR-geometry. Preprint (1999).

[11] M. Chyba, Le cas Martinet en géométrie sous-Riemannienne, Thèse de l'Université de Bourgogne (1997).

[12] H. Davis, Introduction to non linear differential and integral equations, Dover, New-York, (1962).

[13] J. Dieudonné, Calcul infinitésimal, Hermann, Paris (1980).

[14] L.V.D. Dries, A. Macintyre and D. Marker, The elementary theory of restricted analytic fields with exponentiation, Annals of Mathematics 140 (1994) 183-205.

[15] J. Ecalle, Introduction aux fonctions analysables et preuve constructive de la conjecture de Dulac, Hermann, Paris (1992).

[16] G.H. Halphen, Traité des fonctions elliptiques, Gauthier-Villars, Tomes I à IV, Paris (1886).

[17] S. Jacquet, Distance sous-riemannienne et sous analycité. Preprint (1997).

[18] A.G. Khovanskii, Fewnomials, Trans. Math. Monographs 88, (1991) AMS.

[19] I. Kupka, Abnormal extremals. Preprint (1992).

[20] I. Kupka, Géométrie sous-Riemannienne, Séminaire Bourbaki (1996).

[21] D.F. Lawden, Elliptic functions and applications, Springer-Verlag, New-York (1989).

[22] E.B. Lee and L. Markus, Foundations of optimal control theory, John Wiley and Sons, New-York (1967).

[23] S. Lefschetz, Differential equations: geometry theory, Dover, New-York (1977).

[24] M.A. Liapounoff, Problème général de la stabilité du mouvement. Annals of Maths. Studies, Princeton University Press (1947).

[25] J.M. Lion and J.P. Rolin, Théorèmes de préparation pour les fonctions logarithmo-exponentielles. Annales de l'Institut Fourier 47 (1997) 859- 884.

[26] W.S. Liu and H.J. Sussmann, Shortest paths for sub-Riemannian metrics of rank-2 distributions. Memoirs of the Americain Math. Society 118, (1995).

[27] S. Lojasiewicz and H.J. Sussmann, Some examples of reachable sets and optimal cost functions that fail to be subanalytic. SIAM J. Control Optim. 23 (1985) 584-598.

[28] A.E.H. Love, A treatise of the mathematical theory of elasticity, Dover (1944).

[29] R. Montgomery, Abnormal minimizers, SIAM J. Control Optim. 32 (1994) 1605-1620.

[30] A. Mourtada and R. Moussu, Applications de Dulac et applications pfaffiennes. Bulletin SMF 125 (1997) 1-13.

[31] R. Moussu and A. Roche, Théorie de Khovanski et problème de Dulac. Inv. Math. 105 (1991) 431-441. 
[32] R. Roussarie, Bifurcations of planar vector fields and Hilbert's 16th problem, Birkhauser, Berlin (1998).

[33] J.J Stoker, Nonlinear elasticity, Gordon and Breach, London (1968).

[34] R.A. Struble, Nonlinear differential equations, Mac Graw Hill (1962).

[35] J. Tannery and J. Molk, Éléments de la théorie des fonctions elliptiques, Gauthier-Villars, Tomes I à IV, Paris (1896).

[36] E.T. Whittaker and G.N. Watson, A course of modern analysis, Cambridge U. Press, New York (1927). 